\title{
6 Perceptions
}

Ce dernier chapitre porte sur les tests de perception effectués à Paris, en Suisse romande et au Québec dans le but de vérifier les hypothèses, tirées des analyses des voyelles produites par les locuteurs-modèles, par rapport à l'appartenance de différents traits vocaliques aux normes de prononciation de ces trois régions (cf. chapitre 5). La présentation des résultats sera, ici aussi, structurée selon chaque région (cf. 6.1 : Paris ; 6.2 : Suisse romande ; 6.3 : Québec) et, pour chaque trait vocalique analysé, sur la base des trois questions posées dans les tests de perception (cf. 3.5.2) : la question d'évaluation sur la base d'une échelle (0\%-100\%) et sans contexte explicite testant la conception d'une norme stable et indépendante de la situation (cf. question 1), ainsi que celles mentionnant des contextes précis, testant la conception d'une norme plurielle dépendante de la situation (cf. question 2 : prononciation idéale des présentateurs de journaux télévisés ; cf. question 3 : prononciation à enseigner dans les cours de FLE). Par la suite, je proposerai pour chacune des trois régions une discussion (cf. $6.1 .5 ; 6.2 .6 ; 6.3 .9$ ) dans laquelle les résultats touchant aux perceptions seront comparés à ceux touchant aux productions, afin de pouvoir proposer des conclusions empiriquement solides quant à l'appartenance des traits étudiés à la norme respective. Dans un dernier temps, je mettrai en lumière plusieurs réserves nécessaires par rapport aux choix méthodologiques effectués dans le cadre de ces trois tests de perception, qui ont pu avoir une influence sur les résultats (cf. 6.4).

\subsection{Paris}

Dans le cas de Paris, tout d'abord, les tests de perception ont porté sur quatre oppositions tirées de Pustka (2008), Lyche/Østby (2009), Hansen/Juillard (2011) et Hansen (2017) et ayant déjà fait l'objet d'une analyse des productions dans l'usage de locuteurs-modèles dans le chapitre précédent (cf. 5.1). Il s'agit de l'opposition /a/ : /a/ (cf. 6.1.1), des oppositions des voyelles moyennes /e/ : / $/$ / (cf. 6.1.2) et $/ \varnothing /: / œ /$ (cf. 6.1.3) et de celle des voyelles nasales /õ̃/ : / $\tilde{\varepsilon} /$ (cf. 6.1.4).

\subsubsection{Opposition /a/:/a/}

Le premier trait soumis à l'évaluation perceptive des informateurs parisiens est l'opposition /a/ : /a/. Rappelons que les analyses acoustiques des productions de locuteurs-modèles ont pu montrer que les voyelles produites dans pâte et patte se 
chevauchent très majoritairement au niveau des formants F1 et F2 chez ces locuteurs (cf. 5.1.1.1). Cette perspective des productions atteste donc que l'opposition ne peut a priori pas être considérée comme faisant partie de la norme de prononciation parisienne, des résultats qu'il s'agira à présent de vérifier sur la base des évaluations perceptives des 96 informateurs parisiens.

\section{Question 1 : évaluation (sans contexte explicite)}

La première question était conçue comme une évaluation ne prenant volontairement pas en compte quelque contexte normatif que ce soit. Les informateurs étaient invités à évaluer les stimuli entendus sur une échelle allant de $0 \%$ (prononciation très peu correcte) à 100\% (prononciation tout à fait correcte). Les résultats montrent principalement que les informateurs évaluent de façon largement plus positive la variante postérieure [a] (locuteurs « ordinaires » : 89,71\%, écart-type : 13,17\% ; locuteurs-modèles : 93,21\%, écart-type : 7,37\%), la variante antérieure obtenant des scores très faibles (locuteurs « ordinaires » : 17,97\%, écart-type : 13,81\% ; locuteurs-modèles : 37,18\%, écart-type : 17,88\%). L'analyse de variance (ANOVA) indique, pour sa part, que toutes les différences entre ces quatre moyennes sont hautement significatives $(p<0,001)$, mise à part la différence des scores obtenus par les variantes postérieures des locuteurs « ordinaires » et des locuteurs-modèles, qui est également significative, mais à un taux moins élevé $(p<0,05)$ (cf. figure 86).

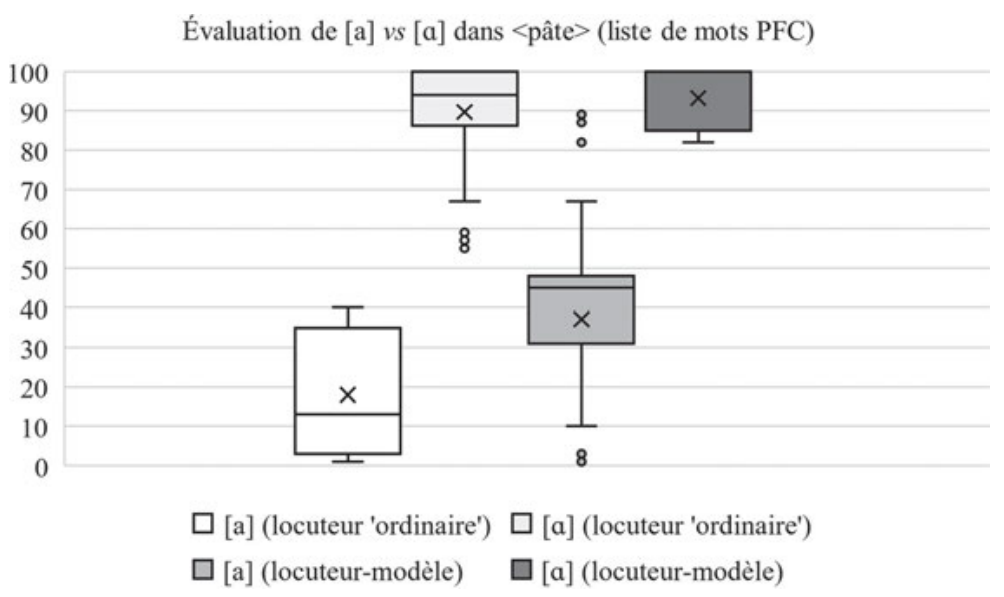

Figure 86 : Évaluation perceptive (en \%) des voyelles [a] et [a] par les informateurs parisiens $(\mathrm{n}=96)$ dans le mot isolé <pâte> (ANOVA : [a] (locuteur « ordinaire ») vs. [a] (locuteur-modèle) : $p<0,05$; autres différences de moyennes : $p<0,001)$. 
Globalement, contrairement à ce que suggéraient les analyses des productions des locuteurs-modèles, ces résultats semblent montrer que la variante postérieure [a] est toujours privilégiée dans le mot pâte dans les perceptions des Parisiens. Un effet du niveau d'éducation des informateurs sur les résultats est par ailleurs relevé par l'analyse de variance : les informateurs sans baccalauréat évaluent en effet la variante antérieure [a] dans le stimulus du locuteur-modèle plus positivement que ceux qui ont le baccalauréat $(F=15,68 ; p<0,001)$. Étant donné qu'il s'agit ici d'évaluations effectuées séparément pour chacune des variantes, c'est-à-dire sans comparaison directe des stimuli, il semblerait que les informateurs plus diplômés (baccalauréat et au-delà) soient légèrement plus enclins à maintenir l'opposition /a/ : / a/ que le groupe d'informateurs dont le niveau scolaire est moins élevé (sans baccalauréat).

Les résultats touchant au stimulus syntagmatique et plus récemment son usine de pâtes italiennes présentent des tendances considérablement différentes : ils ne montrent pas de différence entre les variantes antérieures [a] et postérieures [a], mais entre les lectures de locuteurs non professionnels (moins bien évaluées) et celles de locuteurs-modèles (mieux évaluées). Les deux stimuli des locuteurs-modèles sont en effet mieux évalués (variante antérieure /a/ : 88,75\%, écart-type : 13,21\% ; variante postérieure /a/ : 88,19\%, écart-type : 18,92\%) que ceux des locuteurs " ordinaires » (variante antérieure /a/ : 77,73\%, écart-type : 23,38\% ; variante postérieure /a/ : 72,57\%, écart-type : 24,34\%). L'analyse de variance confirme ces résultats : elle montre que les évaluations des deux stimuli des locuteurs-modèles ne se différencient pas significativement $(p>0,05)$, ce qui est également le cas des évaluations des deux stimuli des locuteurs « ordinaires » $(p>0,05)$, alors que toutes les autres différences sont hautement significatives $(p<0,001)$. Les facteurs sociodémographiques pris en compte dans cette même analyse (sexe, âge, origine et niveau d'éducation des informateurs) ne révèlent pour leur part aucun effet significatif ( $p>0,05)$ (cf. figure 87 ).

Globalement, les résultats obtenus grâce à ces stimuli syntagmatiques ne confirment donc pas les résultats liés au mot isolé pâte. Leur fiabilité par rapport au trait à évaluer peut cependant être remise en question, étant donné le long contexte linguistique dans lequel se trouve le mot pâte, qui a éventuellement pu troubler les informateurs dans leur discernement du trait à évaluer. Ils indiquent cependant deux tendances intéressantes : tout d'abord, c'est la différence de prononciation entre locuteurs ordinaires et locuteurs-modèles qui semble principalement perçue par les informateurs, la prononciation de locuteurs-modèles étant significativement privilégiée. Il s'agit ainsi d'un premier indice montrant qu'une différence est bel et bien faite entre ces deux groupes de locuteurs dans la perception des auditeurs, les locuteurs-modèles étant davantage perçus comme détenteurs de la norme de prononciation (cf. 3.5.1). Par ailleurs, la perte perceptive de 
Évaluation de [a] $v s[a]$ dans $\langle$ pâte $>$ (texte PFC)

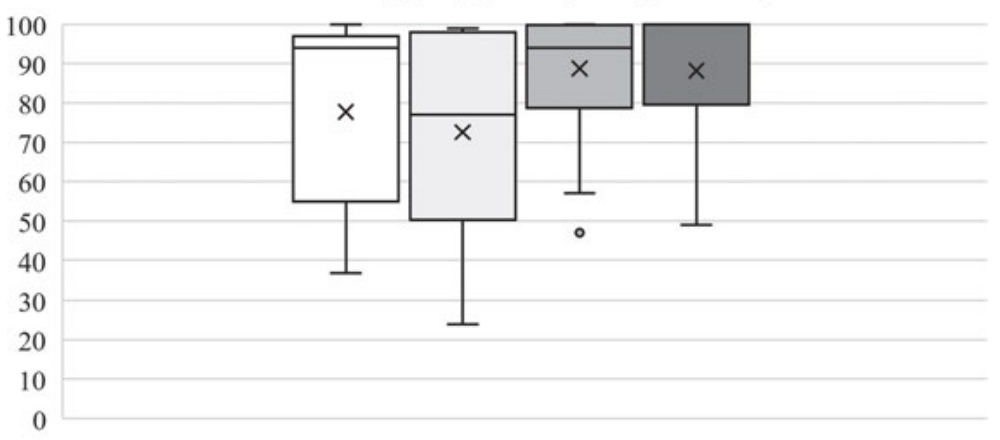

$\square[\mathrm{a}]$ (locuteur 'ordinaire') $\quad \square[\mathrm{a}]$ (locuteur 'ordinaire')
$\square[\mathrm{a}]$ (locuteur-modèle) $\quad \square[\mathrm{a}]$ (locuteur-modèle)

Figure 87 : Évaluation perceptive (en \%) des voyelles [a] et [a] par les informateurs parisiens $(\mathrm{n}=96)$ dans le mot <pâte> tiré du texte PFC (ANOVA : [a] (locuteurs « ordinaire ») vs. [a] (locuteur « ordinaire ») : $p>0,05$; [a] (locuteur-modèle) vs. [a] (locuteur-modèle) : $p>0,05$; autres différences de moyennes : $p<0,001)$.

la différence entre [a] et [a] dans le reste du stimulus syntagmatique pourrait indiquer que cette opposition n'est pas particulièrement saillante dans la perception des informateurs parisiens.

\section{Question 2 : prononciation idéale des présentateurs de journaux télévisés}

La deuxième question, par laquelle les informateurs étaient invités à indiquer la prononciation qu'ils estiment idéale pour des présentateurs de télévision parisiens, confirme la tendance globale déjà observée : une forte majorité de $67,71 \%$ (65/96) des informateurs préfèrent en effet la variante postérieure [a] du locuteur-modèle, contre 27,08\% (26/96) d'entre eux qui optent pour cette même variante postérieure chez le locuteur « ordinaire » et 5,21\% (5/96) seulement qui privilégient la variante antérieure [a] du locuteur-modèle, ces différences étant hautement significatives $(p<0,001)$. En revanche, le modèle de régression appliqué aux données brutes ne montre aucun effet significatif des variables sociodémographiques (sexe, âge, origine, niveau d'éducation) (cf. figure 88).

Les résultats liés au syntagme, pour leur part, confirment globalement les tendances mises en évidence sur la base du mot isolé : une large majorité des informateurs privilégie la variante avec [a] postérieur du locuteur-modèle $(71,88 \%, 69 / 96)$, alors qu'une petite minorité préfère la variante avec [a] antérieur de ce même locuteur-modèle $(28,13 \%, 27 / 96)$, aucun informateur n'ayant choisi les variantes du locuteur « ordinaire» $(p<0,001)$. Le modèle de régression montre 
Selon vous, comment un présentateur de télévision parisien devrait-il prononcer le mot suivant? «pâte »

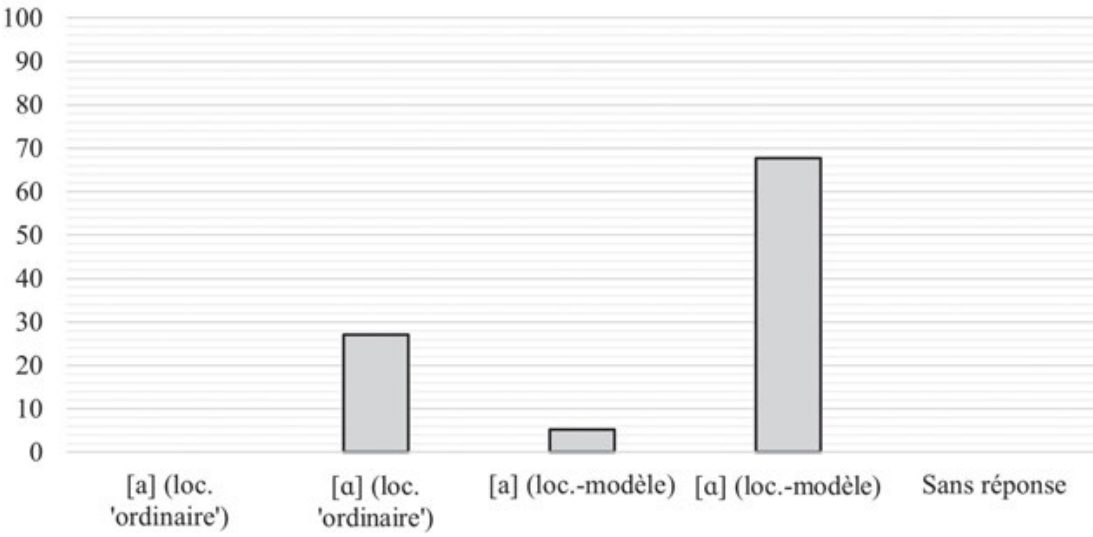

Figure 88 : Voyelle privilégiée (en \%) dans le mot <pâte> pour les présentations de journaux télévisés $(\mathrm{n}=96)$ - Mots isolés ( $p<0,001$, coef. $=2,77$, e.s. $=0,41, \mathrm{t}=6,80)$.

par ailleurs à nouveau un effet significatif du niveau d'éducation sur les résultats $(p<0,01)$, les informateurs au niveau d'éducation plus faible ayant plus souvent tendance à choisir la variante antérieure [a] $(35,42 \%, 17 / 48)$ que les informateurs dont le niveau d'éducation est plus élevé $(20,83 \%, 10 / 48)$ (cf. figure 89$)$.

Ainsi, en plus de la confirmation de la large préférence des informateurs pour la variante postérieure [a] et de celle, plus globale, de la prononciation de locuteursmodèles par rapport à celle de locuteurs " ordinaires », les tendances liées au facteur du niveau d'éducation sont similaires à celles mises en lumière dans la première question : il semble que les informateurs plus diplômés (titulaires du baccalauréat) soient effectivement légèrement plus enclins à percevoir l'opposition $/ \mathrm{a} /$ : /a/. En d'autres termes, chez ces informateurs, la saillance de l'opposition paraît en partie liée à leur niveau d'éducation. Les résultats montrent d'ailleurs indirectement que cette saillance est très faible d'un point de vue général : en effet, seule la comparaison directe des stimuli, comme ici, semble permettre aux informateurs de percevoir la différence entre les variantes antérieure et postérieure.

\section{Question 3 : prononciation à enseigner dans les cours de français langue étrangère (FLE)}

Finalement, la troisième question, dans laquelle il s'agissait de choisir la meilleure prononciation à enseigner dans des cours de FLE, montre pour le mot isolé pâte des tendances quasiment identiques à celles trouvées dans le cas de la deuxième question : 84,38\% (81/96) des informateurs privilégient la variante postérieure [a] 
Selon vous, comment un présentateur de télévision parisien devrait-il prononcer

\section{[a] (loc. 'ordinaire') \\ [a] (loc.-modèle) [a] (loc.-modèle) \\ [a] (loc. 'ordinaire') \\ Sans réponse}

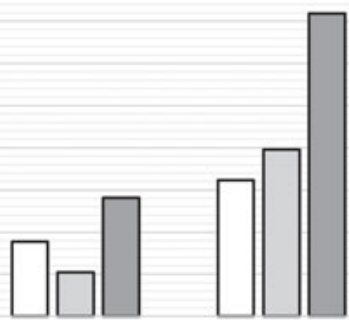

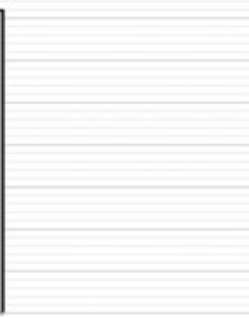

口Sans baccalauréat $\square$ Avec baccalauréat $\square$ Total

Figure 89 : Voyelle privilégiée (en \%) dans le mot <pâte> pour les présentations de journaux télévisés $(\mathrm{n}=96)$ - Texte PFC ; réponses présentées dans leur globalité $(p<0,001$, coef. $=3,71$, e.s. $=0,18, t=20,69)$ ainsi que selon le niveau d'éducation des informateurs : $(p<0,01$, coef. $=0,23$, e.s. $=0,08, t=2,74$ ).

du locuteur-modèle, 11,46\% (11/96) cette même variante postérieure chez le locuteur « ordinaire » et 4,17\% (4/96) la variante antérieure du locuteur-modèle, les différences entre ces taux étant hautement significatives $(p<0,001)$. Le modèle de régression ne montre aucun effet significatif des variables sociodémographiques $(p>0,05)$ (cf. figure 90).

Ces tendances sont corroborées par les résultats du syntagme et plus récemment son usine de pâtes italiennes : en effet, 95,38\% (92/96) des informateurs choisissent à nouveau la variante postérieure [a] du locuteur-modèle, contre 4,17\% préférant la variante antérieure [a] de ce même locuteur, cette différence étant significative à $p<0,001$ (cf. figure 91).

Bien que cette observation soit peu fiable en raison de la faiblesse du nombre absolu d'occurrences, on remarquera que les quatre mentions de la variante antérieure [a] sont attribuables à des informateurs peu diplômés (sans baccalauréat) $(8,33 \%, 4 / 48)$, aucun informateur dont le niveau d'éducation est plus élevé n'ayant choisi cette variante $(0,00 \%, 0 / 48)$. Le modèle de régression montre d'ailleurs que cet effet de l'éducation est légèrement significatif ( $p<0,05$, coef. $=0,08$, e.s. $=0,04, t=2,13)$. Même s'il ne concerne que 4 occurrences, ce résultat - qui converge avec ceux des deux autres questions liées au syntagme - confirme donc que la saillance de l'opposition semble bien être liée au niveau d'éducation des informateurs. 
Selon vous, laquelle des prononciations du mot suivant serait la plus adaptée à être enseignée à des non-francophones ? « pâte »

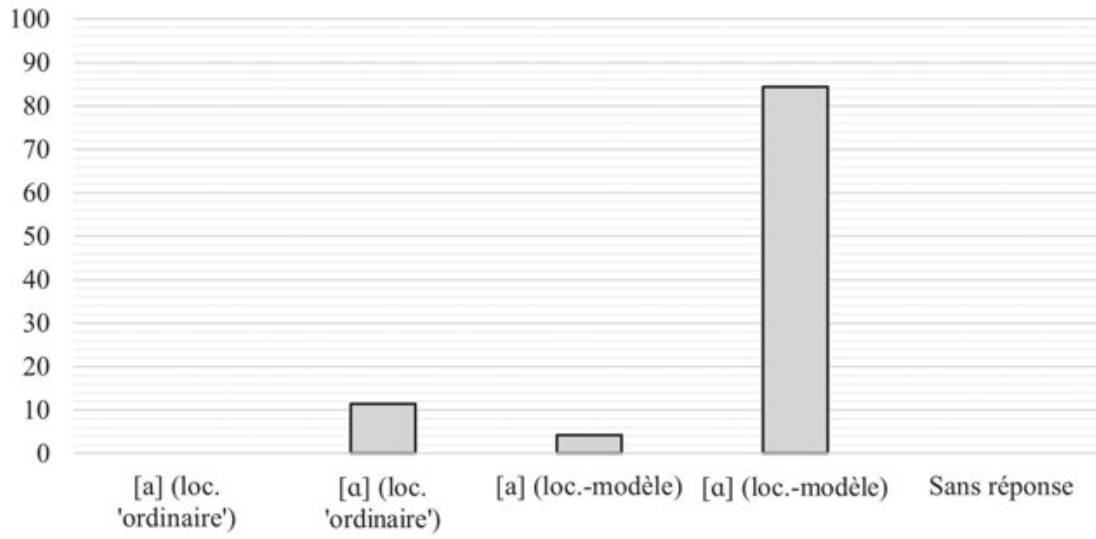

Figure 90 : Voyelle privilégiée (en \%) dans le mot <pâte> pour les cours de français langue étrangère $(\mathrm{FLE})(\mathrm{n}=96)$ - Mots isolés $(p<0,001$, coef. $=4,25$, e.s. $=0,27, t=15,89)$.

Selon vous, laquelle des prononciations du groupe de mots suivant serait la plus adaptée à être enseignée à des non-francophones ? « Et plus récemment son usine de pâtes italiennes "

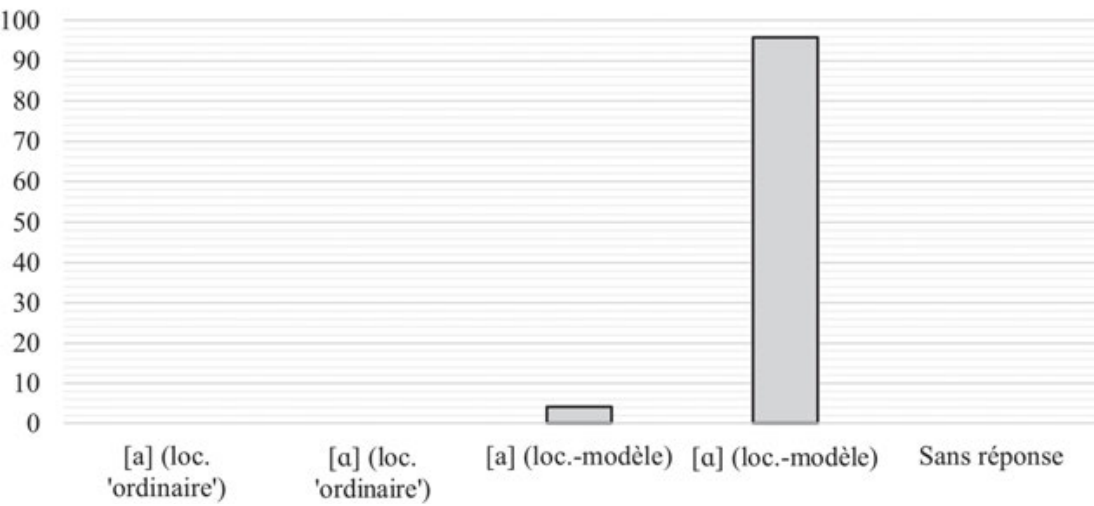

Figure 91 : Voyelle privilégiée (en \%) dans le mot <pâte> pour les cours de français langue étrangère (FLE) $(\mathrm{n}=96)$ - Texte PFC ( $p<0,001$, coef. $=0,95$, e.s. $=0,09, t=11,13)$.

En conclusion, deux principales observations peuvent être retenues de ces résultats : tout d'abord, ils contredisent les analyses des productions des locuteursmodèles en ce sens qu'ils semblent montrer que l'opposition existe encore bel et bien dans les perceptions des informateurs. Cependant, les différences observées entre les résultats touchant aux mots isolés et aux syntagmes suggèrent qu'il pourrait s'agir d'une différence liée non pas uniquement aux perceptions réelles (impli- 
cites) des informateurs, mais à leurs représentations (plus explicites) (cf. 3.1 ; cf. également Lippi-Green 2012 ; Moyer 2013 ; Didelot 2019). En effet, l'opposition n'étant identifiée que dans des mots isolés et/ou des comparaisons directes de stimuli, son degré de saillance semble trop faible pour être systématiquement perceptible, de sorte que seuls des contextes très explicites faisant plutôt appel à la connaissance de l'opposition dans les représentations permettent de la percevoir. De futures études devront donc encore montrer dans quelle mesure cette persistance est bien de nature perceptive, ou déterminer si elle est plutôt due à de simples représentations explicites de l'opposition. Cette dernière interprétation semble d'autant plus plausible que le niveau d'études des informateurs semble jouer un rôle dans la perception de cette opposition, les informateurs chez lesquels il est plus élevé semblant plus enclins à la détecter que ceux dont le niveau d'éducation est moins élevé.

\subsubsection{Opposition $/ e /: / \varepsilon /$}

Le deuxième trait soumis aux évaluations perceptives concerne l'opposition des voyelles moyennes /e/ : / $/$ /. L'analyse des productions a globalement pu montrer une tendance des locuteurs-modèles à la neutralisation de cette opposition en faveur d'un [e] en syllabe ouverte, mais également une variation inter-individuelle et lexicale considérable dans la réalisation $d u / \varepsilon /$ dans ce contexte. Dans la paire minimale épais épée, la tendance observée était au maintien, alors que dans les paires minimales et $\sim$ est et été $\sim$ étaient, elle était à la neutralisation de cette même opposition. Ces résultats seront mis ci-dessous à l'épreuve des données de perceptions.

\section{Question 1 : évaluation (sans contexte explicite)}

Les résultats de la première question touchant tout d'abord au mot isolé épais montrent des tendances allant clairement en direction d'une préférence pour la variante ouverte $[\varepsilon]$ : cette variante obtient en effet des scores très élevés dans l'usage du locuteur " ordinaire » (92,49\%, écart-type : 8,04\%) et du locuteurmodèle $(94,84 \%$, écart-type : 5,61\%), alors que la variante [e] présente des scores faibles et une grande dispersion des données aussi bien dans le cas du locuteur « ordinaire » (38,97\%, écart-type : 30,07\%) que dans celui du locuteur-modèle (40,54\%, écart-type : 29,06\%). L'analyse de variance confirme ces tendances en indiquant des différences hautement significatives entre ces deux groupes de variantes ( $p<0,001)$, alors que les évaluations des deux variantes fermées [e] ne se distinguent pas l'une de l'autre $(p>0,05)$ et que celles des variantes ouvertes $[\varepsilon]$ se distinguent significativement, mais à un niveau plus faible $(p<0,05)$ (cf. figure 92). 


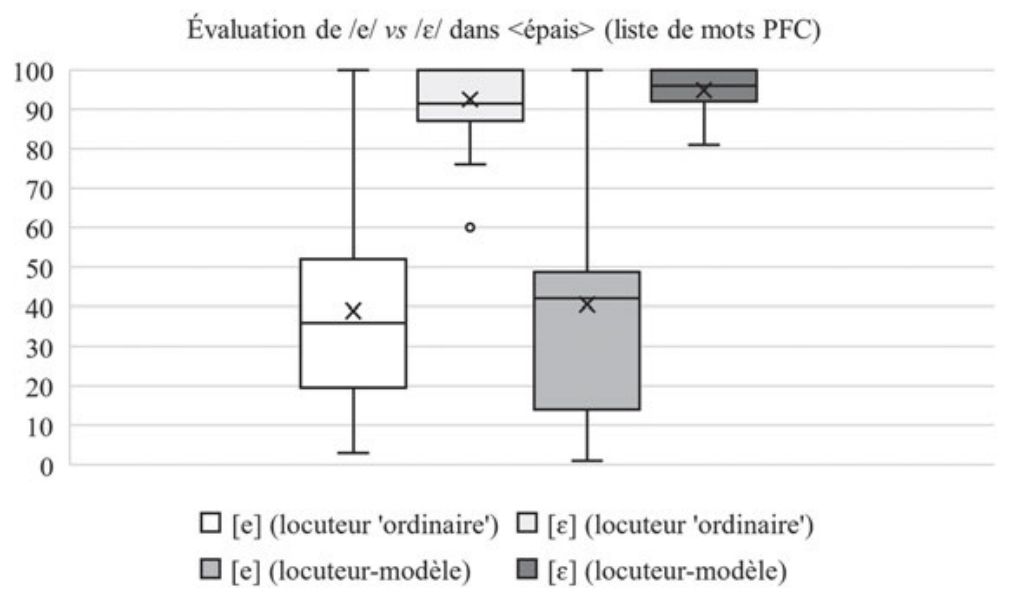

Figure 92 : Évaluation perceptive (en \%) des voyelles [e] et [ $\varepsilon$ ] par les informateurs parisiens $(\mathrm{n}=96)$ dans le mot isolé <épais> (ANOVA : [ $\varepsilon$ ] (locuteur " ordinaire ») vs. [ $\varepsilon$ ] (locuteurs-modèles) : $p<0,05$; [e] (locuteur « ordinaire ») vs. [e] (locuteurs-modèles) : $p>0,05$; autres différences de moyennes : $p<0,001)$.

Notons également l'effet significatif du niveau d'éducation sur les stimuli du locuteur-modèle ([e] $: F=5,52, p<0,05 ;[\varepsilon]: F=4,87, p<0,05$ ), les informateurs sans baccalauréat ayant tendance à évaluer de manière légèrement plus positive sa variante fermée [e] et légèrement plus négative sa variante ouverte $[\varepsilon]$. Étant donné que dans cette question, les stimuli n'ont pas été comparés directement les uns aux autres, il semble donc que la saillance de l'opposition /e/ : / $\varepsilon$ / soit, ici aussi, en partie liée au niveau d'études des informateurs.

Les résultats touchant aux variantes de est dans le syntagme le village de Beaulieu est en grand émoi sont, pour leur part, globalement très ambigus. Tout d'abord, de manière similaire au cas du syntagme pris en compte pour tester l'opposition /a/: /a/, l'évaluation de ce stimulus montre surtout une différence entre les évaluations des stimuli des locuteurs « ordinaires » ([e] : 84,61\%, écart-type : 20,19\% ; $[\varepsilon]: 90,21 \%$, écart-type : $15,18 \%$ ) et des locuteurs-modèles ([e] : 94,04\%, écart-type : $6,10 \% ;[\varepsilon]: 93,80 \%$, écart-type : 7,86\%), les stimuli de ces derniers étant globalement privilégiés. Les moyennes obtenues par les variantes respectives de ces deux groupes sont en effet significativement différentes à $p<0,05$ au moins (cf. figure 93).

L'indice découvert dans le cas de l'opposition /a/ : /a/ (cf. 6.1.1) montrant un comportement évaluatif différent des informateurs par rapport à ces deux groupes de locuteurs semble donc se confirmer ici. Par ailleurs, une différence semble être faite entre la variante fermée [e] et la variante ouverte $[\varepsilon]$ chez les locuteurs ordinaires, leurs scores étant significativement différents à $p<0,05$. On ne retrouve cependant pas la même différence chez les locuteurs-modèles $(p>0,05)$. Il existe 
Évaluation de /e/ vs / $/$ dans $<$ est $>$ (texte PFC)

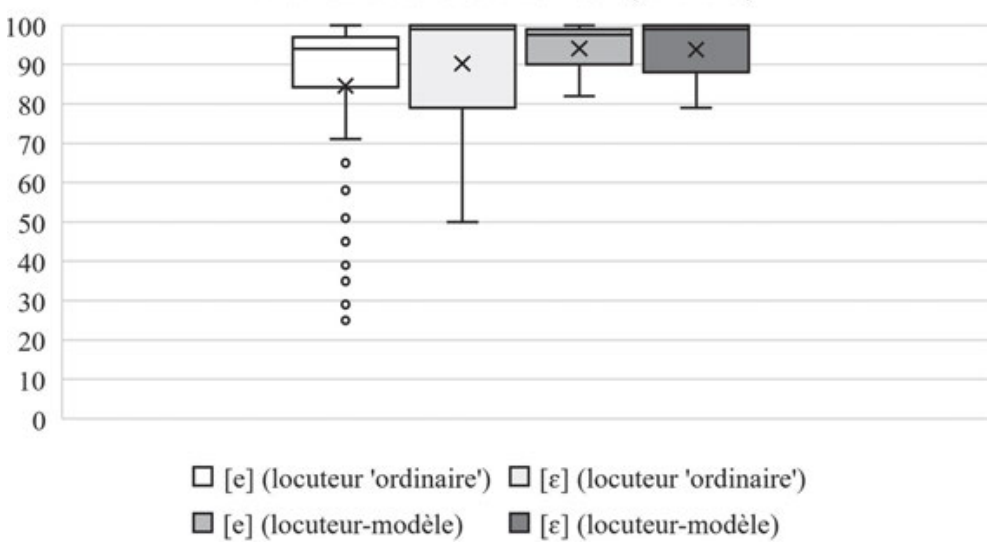

Figure 93 : Évaluation perceptive (en \%) des voyelles [e] et [ $\varepsilon$ ] par les informateurs parisiens $(\mathrm{n}=96)$ dans le mot <est> tiré du texte PFC (ANOVA : [e] vs. [ $\varepsilon]$ (locuteur « ordinaire ») : $p<0,05$;

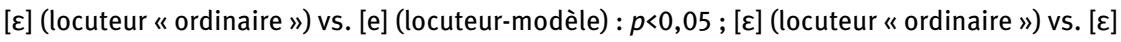
(locuteur-modèle) : $p<0,05 ;$ [e] (locuteur-modèle) vs. [ $\varepsilon$ ] (locuteur-modèle) : $p>0,05)$; autres différences de moyennes : $p<0,001)$.

ainsi une certaine tendance, bien que faible, à préférer la variante ouverte $[\varepsilon]$. Mais dans un stimulus syntagmatique plus long tel qu'ici, la différence semble disparaître perceptivement dans le contexte linguistique du stimulus. Ceci plaide donc également en faveur d'un manque de saillance de l'opposition dans la perception des informateurs. Finalement, un résultat secondaire peut à nouveau être noté par rapport au facteur de l'éducation : en effet, ce facteur montre à nouveau un effet significatif sur les évaluations des deux stimuli du locuteur-modèle ([e] : $F=7,21, p<0,01 ;[\varepsilon]: F=9,31, p<0,01):$ les informateurs au niveau d'éducation moins élevé (sans baccalauréat) évaluent de manière légèrement plus positive la variante fermée [e] et légèrement plus négative la variante ouverte $[\varepsilon]$ que les informateurs ayant un niveau d'éducation plus élevé (titulaires du baccalauréat).

Les résultats des évaluations des variantes de étaient dans le syntagme jusqu'ici les seuls titres de gloire de Beaulieu étaient son vin blanc sec montrent globalement que la variante ouverte $[\varepsilon]$ du locuteur-modèle est préférée. Elle se différencie en effet significativement des trois autres $(p<0,001)$, alors que ces dernières ne se distinguent pas significativement entre elles ( $p>0,05)$ (cf. figure 94).

Les moyennes relevées indiquent par ailleurs, ici aussi, une tendance à mieux évaluer dans l'ensemble les stimuli des locuteurs-modèles. Par ailleurs, les écart-types, très élevés, suggèrent une certaine ambiguïté dans l'évaluation de ce stimulus. Le manque de saillance de l'opposition observé dans le premier syn- 
Évaluation de /e/ vs / $/$ dans <étaient $>$ (texte PFC)

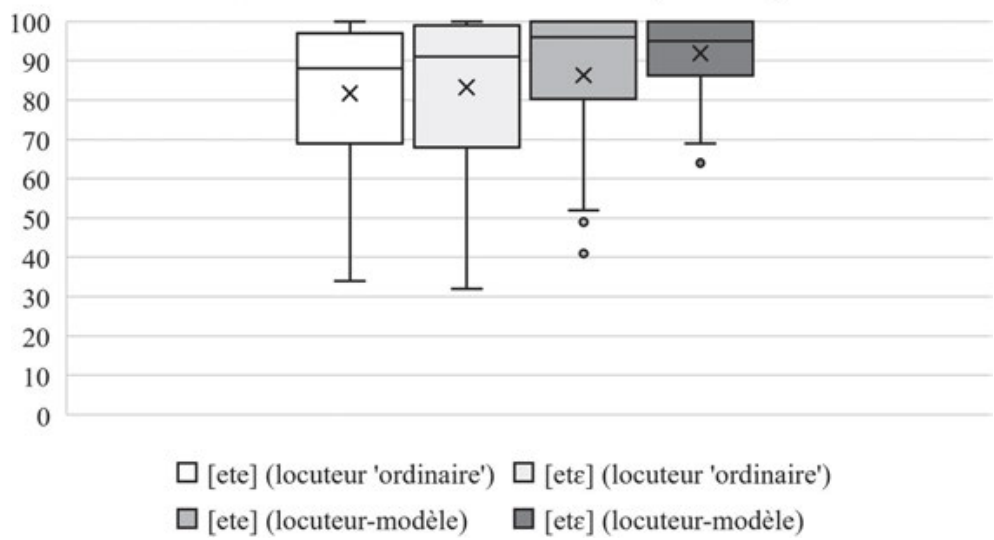

Figure 94 : Évaluation perceptive (en \%) des voyelles [e] et [ $[\varepsilon]$ par les informateurs parisiens $(\mathrm{n}=96)$ dans le mot <étaient> tiré du texte PFC (ANOVA : [e] vs. [ $\varepsilon]$ (locuteur « ordinaire ») : $p>0,05 ;[e]$ (locuteur « ordinaire ») vs. [e] (locuteur-modèle) : $p>0,05 ;[\varepsilon]$ (locuteur " ordinaire ») vs. [e] (locuteur-modèle) : $p>0,05$; autres différences de moyennes : $p<0,001$ ).

tagme (cf. supra) semble donc se confirmer ici. Quant aux facteurs pris en compte (sexe, âge, origine, niveau d'éducation), ils ne s’avèrent pas significatifs ( $p>0,05)$.

\section{Question 2 : prononciation idéale des présentateurs de journaux télévisés}

Les résultats touchant à la deuxième question, dans laquelle les stimuli sont contrairement à la première question - comparés directement, indiquent pour leur part deux tendances relativement claires : d'une part, la variante ouverte $[\varepsilon]$ est fortement privilégiée par rapport à la variante fermée [e]. D’autre part, les informateurs présentent une nette préférence pour cette variante $[\varepsilon]$ chez les locuteursmodèles. En effet, 67,71\% (65/96) des informateurs portent leur choix sur le $[\varepsilon]$ du locuteur-modèle, alors que seuls 19,79\% (19/96) d'entre eux préfèrent cette même variante chez le locuteur « ordinaire ». Le modèle de régression indique que cette différence est significative à $p<0,001$ (cf. figure 95). Précisons que cette différence entre locuteurs-modèles et locuteurs ordinaires n'a été établie qu'après coup dans le cadre de la présentation des résultats, les informateurs n'ayant fait qu'écouter les quatre stimuli et cliquer sur le stimulus qu'ils préfèrent, sans avoir reçu une quelconque information sur ces locuteurs.

Les cas du mot est dans le premier stimulus syntagmatique (le village de Beaulieu est en grand émoi) est très similaire, avec une variante ouverte $[\varepsilon]$ chez le locuteur-modèle $(80,21 \%, 77 / 96)$ privilégiée de manière hautement significative $(p<0,001)$. Relevons également que les informateurs portent plus souvent leur 
Selon vous, comment un présentateur de télévision parisien devrait-il prononcer le mot suivant ? « épais »

100
90
80
70
60
50
40
30
20
10
0
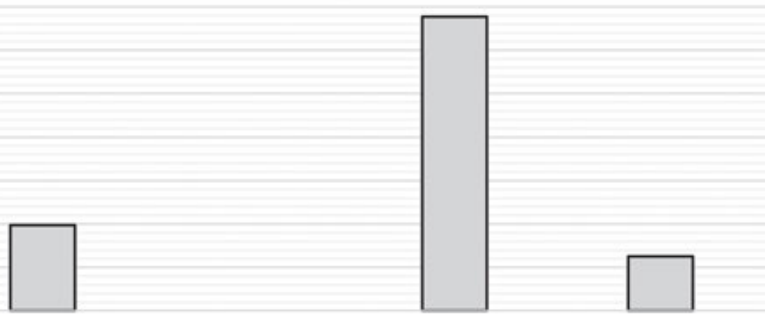

[e] (loc. 'ordinaire')

[ع] (loc. 'ordinaire')

[e] (loc.-modèle) [ $[\varepsilon]$ (loc.-modèle)

Sans réponse

Figure 95 : Voyelle privilégiée (en \%) dans le mot <épais> pour les présentations de journaux télévisés $(\mathrm{n}=96)$ - Mots isolés ( $p<0,001$, coef. $=4,03$, e.s. $=0,38, t=10,68)$.

choix sur la variante fermée [e] du locuteur-modèle $(14,58 \%, 14 / 96)$ que sur la variante ouverte $[\varepsilon]$ du locuteur « ordinaire » $(4,17 \%, 4 / 96)$ (cf. figure 96$)$.

Selon vous, comment un présentateur de télévision parisien devrait-il prononcer

le groupe de mots suivant ? «Le village de Beaulieu est en grand émoi »

$$
\begin{array}{r}
100 \\
90 \\
80 \\
70 \\
60 \\
50 \\
40 \\
30 \\
20 \\
10 \\
0
\end{array}
$$

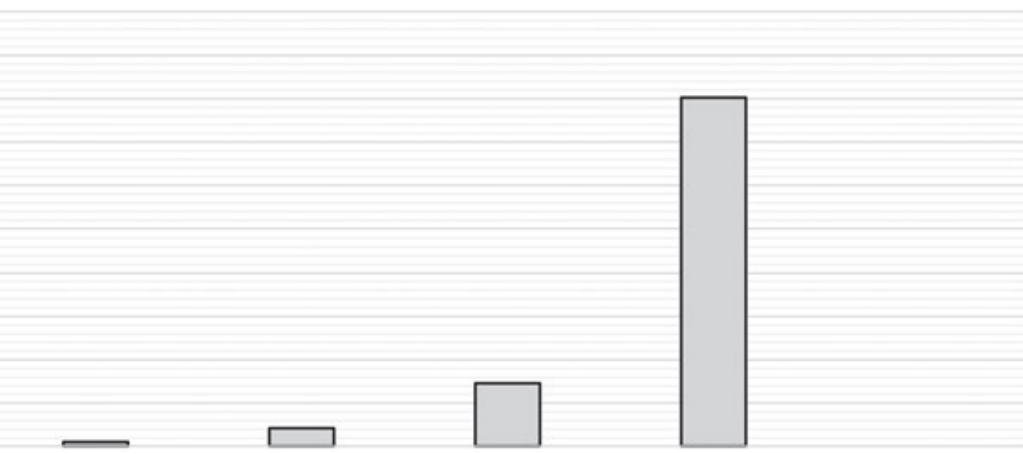

[e] (loc. 'ordinaire')

[ع] (loc. 'ordinaire') [e] (loc.-modèle) $[\varepsilon]$ (loc.-modèle) Sans réponse

Figure 96 : Voyelle privilégiée (en \%) dans le mot <est> pour les présentations de journaux télévisés $(\mathrm{n}=96)$ - Texte PFC $(p<0,001$, coef. $=3,07$, e.s. $=0,27, t=12,74)$.

Les observations sont très similaires pour les résultats obtenus par étaient dans le deuxième syntagme (jusqu'ici les seuls titres de gloire de Beaulieu étaient son 
vin blanc sec), avec une préférence hautement significative $(p<0,001)$ pour cette même variante $([\varepsilon]$ du locuteur-modèle : 54,17\%, 52/96) et, à nouveau, une préférence pour le $[\mathrm{e}] \mathrm{du}$ locuteur-modèle $(27,08 \%, 26 / 96)$ par rapport au $[\varepsilon] \mathrm{du}$ locuteur « ordinaire » $(7,29 \%, 7 / 96)$ (cf. figure 97$).$

Selon vous, comment un présentateur de télévision parisien devrait-il prononcer le groupe de mots suivant? « Jusqu'ici les seuls titres de gloire de Beaulieu étaient son vin blanc $\mathrm{sec}$ »

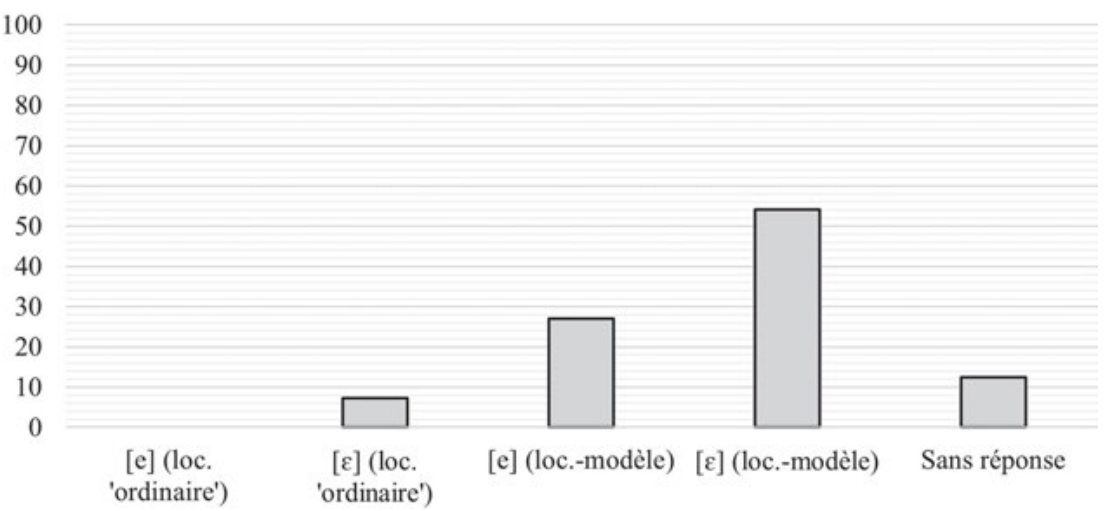

Figure 97 : Voyelle privilégiée (en \%) dans le mot <étaient> pour les présentations de journaux télévisés $(\mathrm{n}=96)$ - Texte PFC ( $p<0,001$, coef. $=3,37$, e.s. $=0,39, t=8,69)$.

Ainsi, les deux stimuli syntagmatiques ne font que confirmer les principales tendances générales observées plus haut, à savoir la préférence des informateurs pour la variante ouverte $[\varepsilon]$ (dans est et étaient) et leur préférence pour les stimuli de locuteurs-modèles. Notons que dans cette question en particulier, les représentations des informateurs pourraient avoir influencé les évaluations perceptives (cf. 3.1) : il est en effet possible que les auditeurs non experts n'aient pas évalué les traits segmentaux entendus mais bien la production globale. Ce faisant, il est envisageable qu'ils aient simplement reconnu chez les locuteurs-modèles, d'une part, une compétence en lecture correspondant à leurs attentes envers la lecture à voix haute d'un professionnel de la parole et, d'autre part, des traits intonatifs correspondant à leurs attentes, les syntagmes étant assez longs pour permettre une différenciation intonative entre présentateurs et locuteurs non experts.

\section{Question 3 : prononciation à enseigner dans les cours de français langue étrangère (FLE)}

Les résultats touchant à dernière question (FLE) ne se différencient pas notablement de ceux concernant les présentateurs de télévision (cf. supra) : dans le cas 
du mot isolé épais, c'est à nouveau la variante ouverte $[\varepsilon]$ du locuteur-modèle qui est privilégiée par 72,29\% (70/96) des informateurs, ce taux étant significativement plus élevé ( $p<0,001)$ que celui de toutes les autres variantes (cf. figure 98).

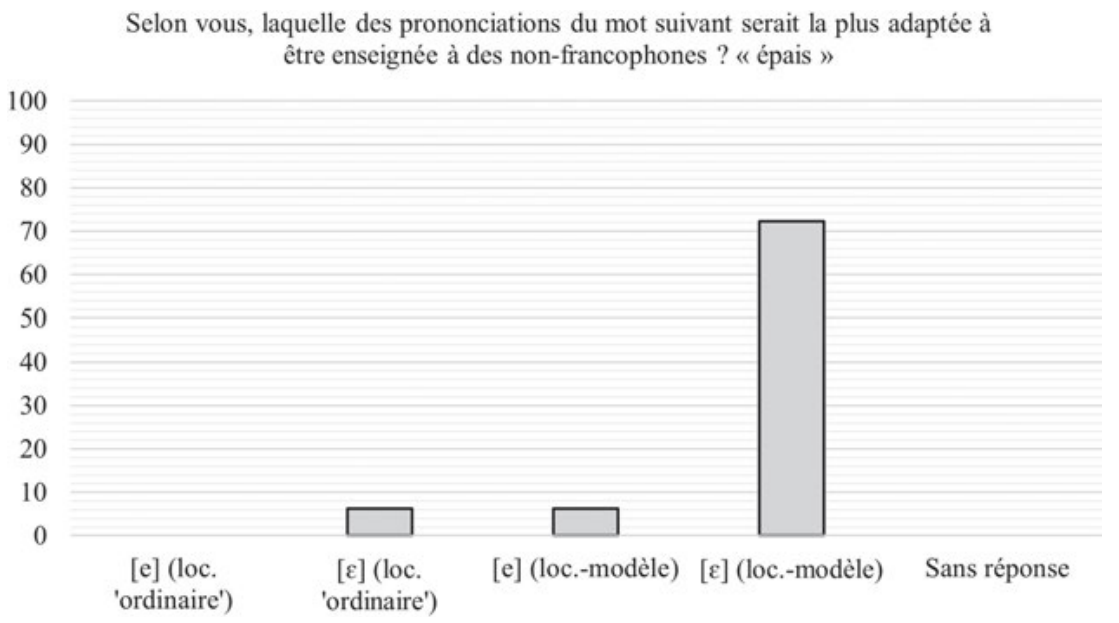

Figure 98 : Voyelle privilégiée (en \%) dans le mot <épais> pour les cours de français langue étrangère (FLE) $(\mathrm{n}=96)$ - Mots isolés ( $p<0,001$, coef. $=2,61$, e.s. $=0,60, t=4,33)$.

Il en va de même pour le syntagme dans lequel les variantes de prononciation du mot est sont testées (le village de Beaulieu est en grand émoi), la variante [ع] du locuteur-modèle étant préférée dans 82,29\% (79/96) des cas, taux significativement plus élevé $(p<0,001)$ que celui de la seule autre variante choisie par les informateurs, celle contenant un [e] fermé chez le locuteur-modèle (17,71\%, 17/96) (cf. figure 99).

La tendance globale par rapport au deuxième syntagme (jusqu'ici les seuls titres de gloire de Beaulieu étaient son vin blanc sec), à l'aide duquel les variantes de étaient devaient être testées, est très similaire : la variante ouverte [ $\varepsilon]$ est préférée par une majorité significative $(p<0,001)$ de 56,25\% (54/96) des informateurs. Cependant, contrairement au cas de la forme est, ce n'est pas le [e] fermé du locuteur-modèle $(17,71 \%, 17 / 96)$ qui arrive en seconde position, mais la variante ouverte $[\varepsilon]$ du locuteur « ordinaire » $(26,04 \%, 25 / 96)$ (cf. figure 100).

Cette dernière observation pourrait indiquer que la variante fermée [e] est perceptivement moins tolérée dans la terminaison de étaient que dans la forme est. Cette observation va, d'une part, à l'encontre des résultats portant sur les productions des locuteurs-modèles, qui n'affichent aucune différence significative de comportement du $/ \varepsilon /$ dans étaient et est (cf. 5.1.1.2) et, d'autre part, dans 
Selon vous, laquelle des prononciations du groupe de mots suivant serait la plus adaptée à être enseignée à des non-francophones ?

« Le village de Beaulieu est en grand émoi »

100

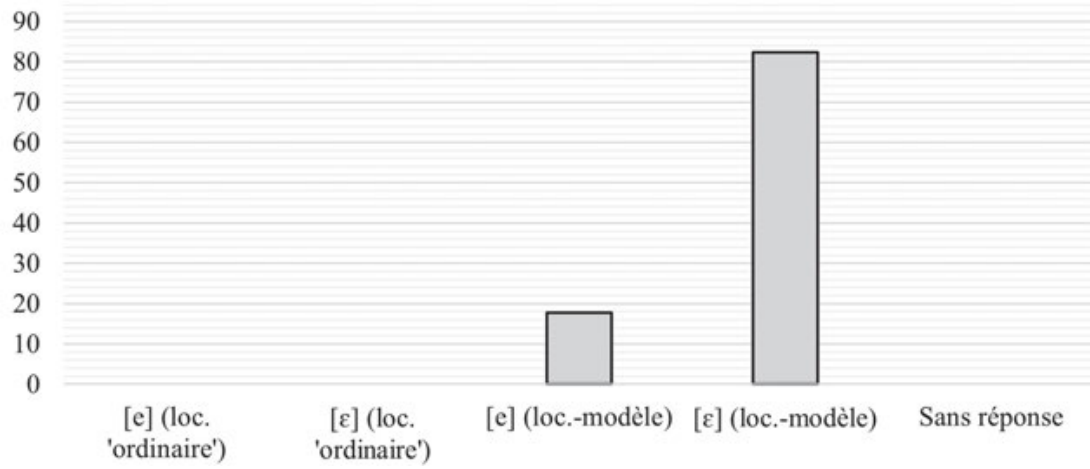

Figure 99 : Voyelle privilégiée (en \%) dans le mot <est> pour les cours de français langue étrangère (FLE) $(\mathrm{n}=96)$ - Texte PFC ( $p<0,001$, coef. $=1,83$, e.s. $=0,14, t=13,31)$.

Selon vous, laquelle des prononciations du groupe de mots suivant serait la plus adaptée à être enseignée à des non-francophones ? « Jusqu'ici les seuls titres de gloire de Beaulieu étaient son vin blanc sec »

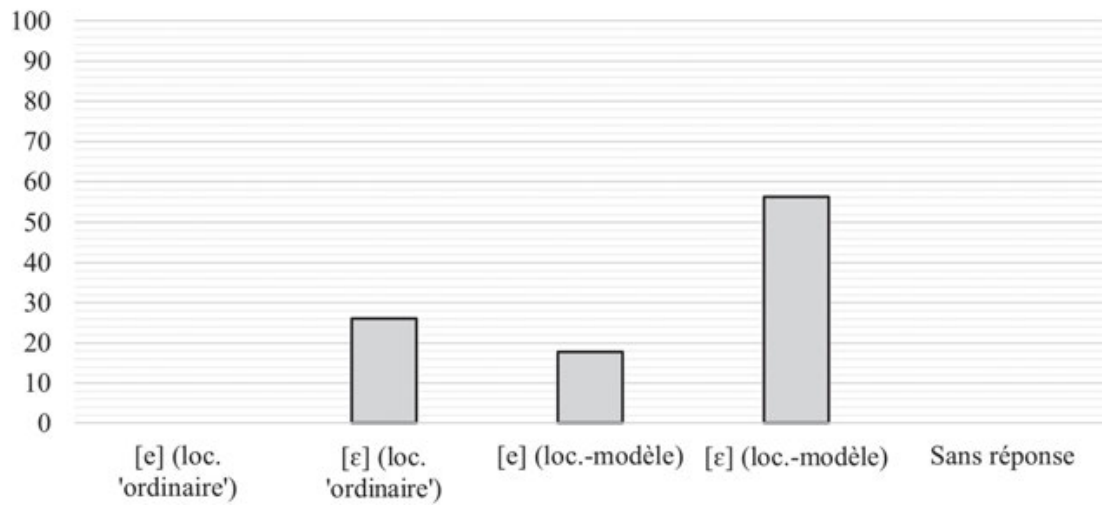

Figure 100 : Voyelle privilégiée (en \%) dans le mot <étaient> pour les cours de français langue étrangère (FLE) $(\mathrm{n}=96)$ - Texte PFC $(p<0,001$, coef. $=1,67$, e.s. $=0,38, t=4,39)$.

la direction des résultats de Lyche/Østby (2009, 212), Hansen/Juillard (2011, 315) et Hansen (2017, 128-129), qui ont montré que la paire minimale été étai(en)t tend à se maintenir, alors que le est tend à la fermeture en [e]. Deux explications de cette différence peuvent être évoquées : tout d'abord, il pourrait s'agir d'une influence de la graphie, les locuteurs ayant davantage tendance à lier la terminai- 
son -aient à un [ع] ouvert (cf. Lyche/Østby 2009, 212). Par ailleurs, cela pourrait aussi résulter de la plus haute fréquence d'apparition de la forme est, qui la rendrait plus sensible au changement. Il ne s'agit cependant ici que d'hypothèses se basant sur un très petit nombre d'occurrences, qu'il faudrait donc vérifier dans de futures études.

En conclusion, on peut tirer le bilan suivant des résultats concernant l'opposition $/ \mathrm{e} / \mathrm{:} / \varepsilon / \mathrm{:}$ premièrement, la préférence pour la variante ouverte $[\varepsilon]$ est

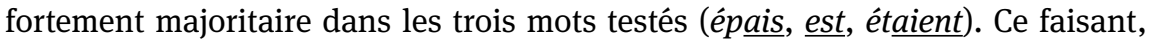
le [e] fermé semble cependant légèrement mieux toléré dans la forme fréquente est. Les résultats de est et étaient vont ainsi à l'encontre des résultats de l'analyse des productions des locuteurs-modèles (cf. 5.1.1.2). On pourrait supposer que les productions se trouvent à un stade plus avancé que les perceptions dans le changement menant à la fermeture du $/ \varepsilon /$ en syllabe finale ouverte. Cependant, il est plus vraisemblable qu'il s'agisse à nouveau d'une différence liée non pas aux perceptions des informateurs, mais à leurs représentations (cf. 3.1). C'est du moins ce que suggèrent les différences trouvées dans la première question entre les résultats touchant aux mots isolés (= contexte dans lequel le trait à évaluer est très explicite et fait par conséquent davantage appel à des représentations) et aux syntagmes (= contexte plus implicite). Il est par ailleurs vraisemblable que les informateurs aient reconnu - pour ce qui est non seulement de la compétence en lecture à haute voix, mais aussi des traits intonatifs - une production globale qu'ils associent à l'usage de présentateurs, et qu'ils l'aient choisi pour cette raison (et ce, pas seulement dans la deuxième question, qui porte explicitement sur les présentateurs). Ceci correspondrait également à une manière d'évaluer faisant appel à des représentations et non à des perceptions. Deuxièmement, les résultats montrent également une forte préférence globale pour les variantes de locuteurs-modèles dans l'optique d'une norme de prononciation. Finalement, la saillance de cette opposition - certes globalement très faible - semble être, à nouveau, légèrement plus haute dans le groupe des informateurs plus diplômés (c'est-à-dire ayant le baccalauréat).

\subsubsection{Opposition /ø/:/œ/}

Le troisième trait soumis aux évaluations perceptives est l'opposition /ø/ : /œ/. Rappelons, d'une part, qu'en français parisien, on observe une tendance croissante de cette opposition à respecter la loi de position, avec un [ø] systématique en syllabe finale ouverte et un [œ] plus courant en syllabe finale fermée (cf. Hansen 2017, 129). D’autre part, les analyses des productions de locuteurs-modèles parisiens ont montré que ces locuteurs continuent à maintenir majoritairement cette 
opposition dans la paire minimale jeune jeûne, même si une tendance considérable à sa neutralisation en faveur d'un unique /œ/ dans cette paire minimale a également pu être révélée (cf. 5.1.1.3). Ces derniers résultats seront vérifiés sur la base des données de perception présentées ci-dessous, ces évaluations perceptives concernant les réalisations fermées et ouvertes du mot jeûne, mot déjà utilisé dans les analyses des productions.

\section{Question 1 : évaluation (sans contexte explicite)}

Les résultats de la première question montrent tout d'abord, par rapport au mot isolé jeûne, que la variante de référence est à nouveau fortement privilégiée par les informateurs : la variante fermée [ø] obtient en effet aussi bien dans le cas du locuteur « ordinaire » (88,44\%, écart-type : 19,46\%) que dans le cas du locuteur-modèle $(97,28 \%$, écart-type : $3,24 \%)$ un score significativement plus élevé $(p<0,001)$ que la variante ouverte [œ] (locuteur « ordinaire » :52,34\%, écarttype : $21,05 \%$; locuteur-modèle : $64,39 \%$, écart-type : 10,01\%). Par ailleurs, on observe également une préférence pour les stimuli des locuteurs-modèles, les différences de scores entre les quatre variantes étant toutes hautement significatives, à $p<0,001$ (cf. figure 101).

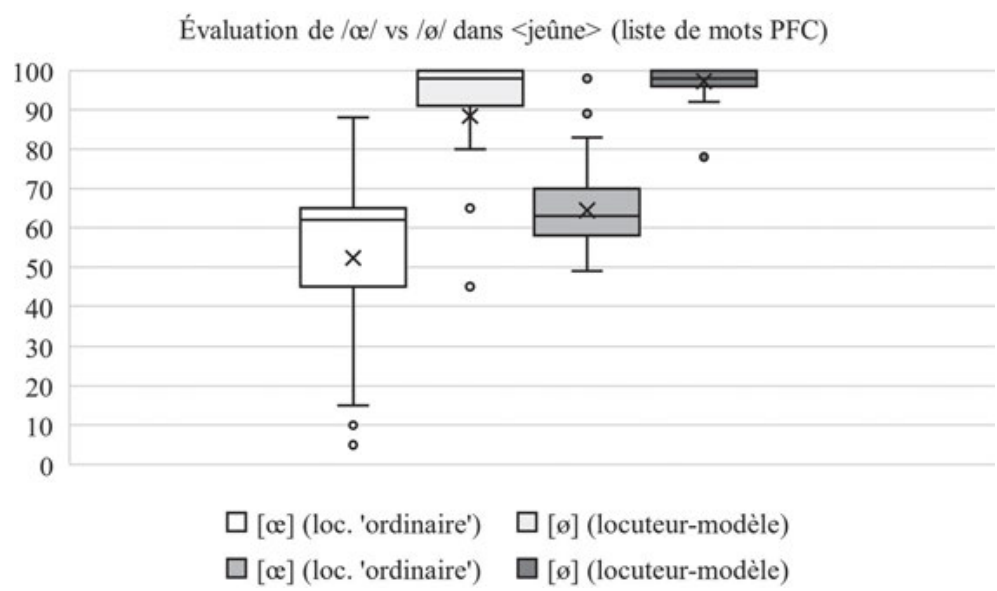

Figure 101 : Évaluation perceptive (en \%) des voyelles [œ] et [ø] par les informateurs parisiens $(\mathrm{n}=96)$ dans le mot isolé <jeûne> (ANOVA : toutes les différences à $p<0,001)$.

L'analyse de variance indique par ailleurs que le facteur du niveau d'éducation a ici aussi un effet significatif sur les résultats des deux variantes des locuteurs-modèles ([œ] locuteur-modèle : $F=23,73, p<0,001$; [ø] locuteur-modèle : $F=4,30, p<0,05$ ). 
Cet effet est similaire à celui qui a été mis en évidence pour les oppositions /a/ : $/ a /$ et $/ e /: / \varepsilon /:$ les informateurs ayant un niveau d'éducation plus élevé (baccalauréat) ont en effet tendance à évaluer de manière nettement plus positive la variante (de référence) fermée [ø] et légèrement plus négative la variante ouverte [œ] que les informateurs dont le niveau d'éducation est moindre (sans baccalauréat).

Les résultats obtenus par le même mot, mais dans un syntagme (Quelques fanatiques auraient même entamé un jeûne prolongé dans l'église de Saint Martinville) sont très similaires, bien qu'ils présentent globalement une plus grande dispersion, observable sur la base de plus grands écart-types : les deux variantes avec [ø] fermé (locuteur « ordinaire » : 87,70\%, écart-type : 11,67\% ; locuteurmodèle : 92,90\%, écart-type : 8,28\%) atteignent en effet des scores significativement plus élevés $(p<0,001)$ que les deux variantes avec [œ] ouvert (locuteur « ordinaire » : 25,90\%, écart-type : 21,21\% ; locuteur-modèle : 34,02\%, écart-type : 19,96\%) (cf. figure 102).

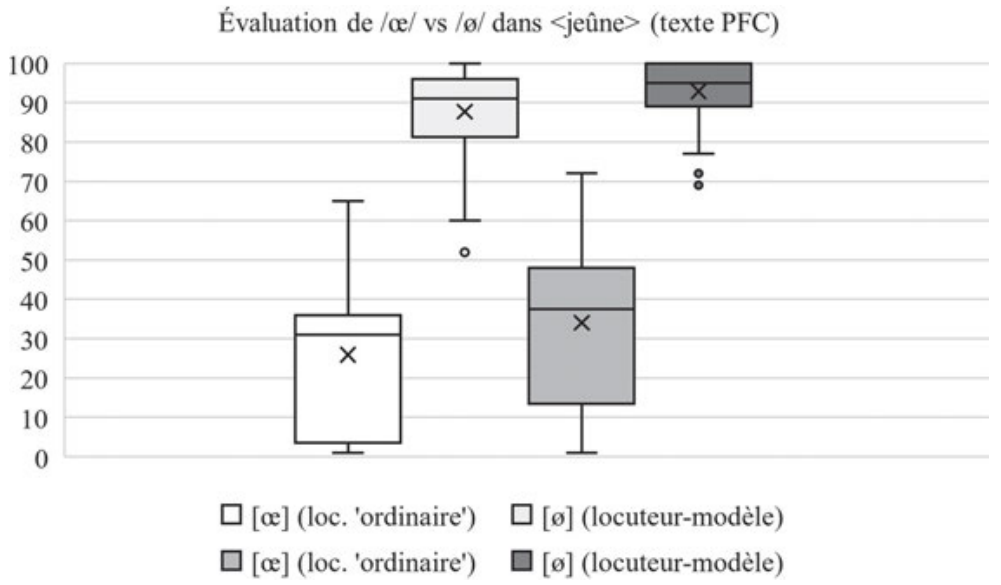

Figure 102 : Évaluation perceptive (en \%) des voyelles [œ] et [ø] par les informateurs parisiens $(n=96)$ dans le mot <jeûne> tiré du texte PFC (ANOVA : $[\varepsilon]$ (locuteur « ordinaire ») vs. $[\varepsilon]$ : $p<0,01$; autres différences de moyennes : $p<0,001)$.

Dans le cas présent également, on relève un effet du niveau d'éducation pour trois des quatre variantes ([œ] locuteur « ordinaire » : $F=4,18, p<0,05 ;[\varnothing]$ locuteur « ordinaire » : $F=14,87, p<0,001$; [ø] locuteur-modèle : $F=27,12, p<0,001)$. Cet effet indique à nouveau que les informateurs plus diplômés (baccalauréat) évaluent plus positivement les variantes fermées [ø] et plus négativement les variantes ouvertes [œ] que les informateurs ayant un niveau d'éducation moins élevé (sans baccalauréat). 
Ainsi, outre une forte tendance à préférer le $[\varnothing]$ dans le contexte du mot jeûne, les résultats de cette première question suggèrent donc également une plus grande tolérance des informateurs peu diplômés (sans baccalauréat) pour la variante ouverte [œ] dans ce contexte.

\section{Question 2 : prononciation idéale des présentateurs de journaux télévisés}

Les résultats globaux de la deuxième question s'avèrent encore plus univoques : la variante avec [ø] fermé est unanimement privilégiée par les informateurs dans l'optique de la présentation de journaux télévisés, 65,63\% (63/96) d'entre eux choisissant cette variante chez le locuteur-modèle et 34,38\% (33/96) chez le locuteur « ordinaire ». Le modèle de régression montre, entre autres, que cette différence est significative à $p<0,001$ (cf. figure 103).

Selon vous, comment un présentateur de télévision parisien devrait-il prononcer le mot suivant ? « jeûne »

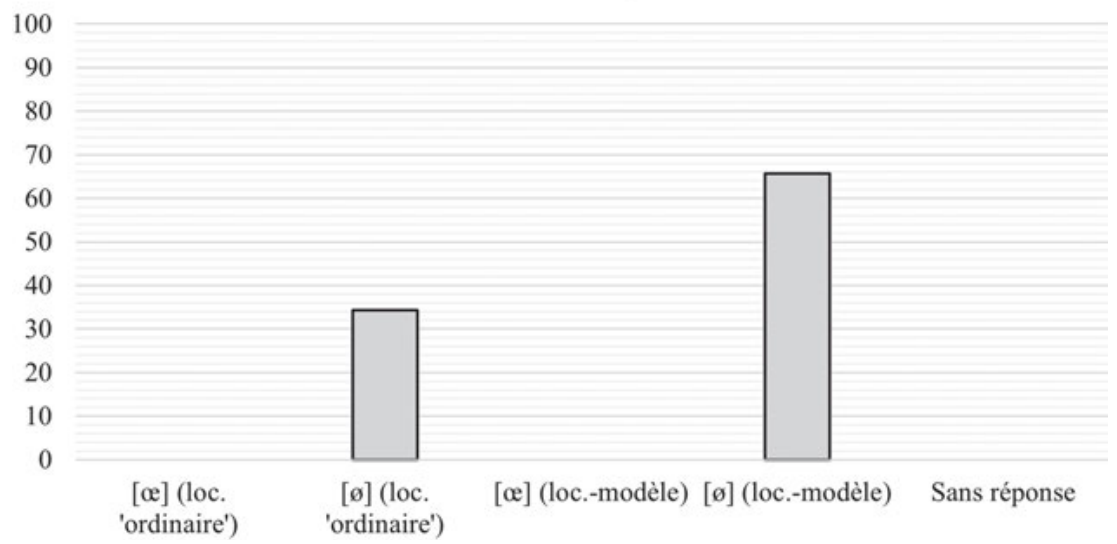

Figure 103 : Voyelle privilégiée (en \%) dans le mot <jeûne> pour les présentations de journaux télévisés $(\mathrm{n}=96)$ - Mots isolés ( $p<0,001$, coef. $=3,85$, e.s. $=0,36, t=10,81)$.

Ainsi, les résultats montrent la préférence unanime des informateurs pour la variante [ø] fermée, et de manière générale, pour la prononciation des locuteurs-modèles, comme c'était déjà le cas des deux oppositions précédentes $(/ \mathrm{a} /$ : /a/ : cf. 6.1.1 ; /e/ : $/ \varepsilon /:$ cf. 6.1.2).

Le résultat obtenu par le stimulus syntagmatique est très similaire, avec une très large préférence pour la variante avec [ø] fermé du locuteur-modèle $(83,33 \%$, 80/96), ce taux étant significativement plus élevé que tous les autres $(p<0,001)$. Cependant, contrairement au cas du mot isolé jeûne, ce n'est pas cette même 
variante fermée chez le locuteur « ordinaire » qui arrive en deuxième position, mais la variante opposée du locuteur-modèle, celle avec [œ] ouvert $(16,67 \%$, 16/96) (cf. figure 104).

Selon vous, comment un présentateur de télévision parisien devrait-il prononcer le mot suivant ? «Quelques fanatiques auraient même entamé un jeûne prolongé dans l'église de Saint Martinville »

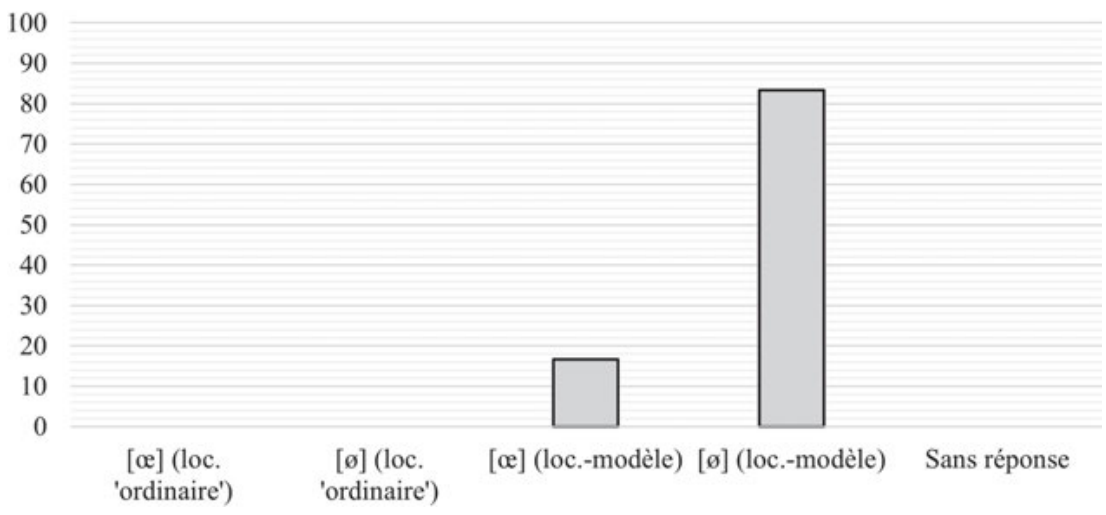

Figure 104 : Voyelle privilégiée (en \%) dans le mot <jeûne> pour les présentations de journaux télévisés $(n=96)$ - Texte PFC $(p<0,001$, coef. $=4,09$, e.s. $=0,15, t=26,40)$.

Comment expliquer cette légère différence entre les variantes arrivant en deuxième position pour le mot isolé et pour le syntagme ? Comme déjà observé dans le cas des oppositions /a/ : /a/ et /e/ : / $/$ /, dans le cadre d'un stimulus plus long, l'opposition à tester semble s'estomper ou, à tout le moins, jouer un rôle moins important que la perception globale du stimulus. L'opposition /ø/ : /œ/ ne paraît donc pas particulièrement saillante dans la perception des informateurs. Et, d'autre part, dans un tel cas, c'est apparemment le type de locuteur qui prime dans les perceptions, les variantes du locuteur-modèle - quelles qu'elles soient étant privilégiées.

Relevons par ailleurs, ici aussi, un très léger effet significatif du niveau d'éducation sur les résultats : les informateurs ayant un niveau plus faible (c'est-à-dire sans baccalauréat) ont en effet à nouveau tendance à choisir un peu plus souvent le stimulus présentant la variante ouverte [œ] $(25,00 \%, 12 / 48)$ que les informateurs titulaires du baccalauréat $(8,33 \%, 4 / 48)$. L'effet n'est cependant que très marginal, étant donné qu'il concerne un très faible nombre d'occurrences et que les informateurs sans baccalauréat, eux aussi, privilégient malgré tout largement le stimulus du locuteur-modèle $(75,00 \%, 36 / 48)$. La plus grande tolérance des 
informateurs au niveau d'éducation moins élevé pour la neutralisation de l'opposition jeune jeûne semble donc se confirmer ici.

\section{Question 3 : prononciation à enseigner dans les cours de français langue étrangère (FLE)}

Finalement, la dernière question, qui touchait à la prononciation-cible pour les cours de FLE indique des résultats peu différents de la deuxième question : dans le cas du mot isolé jeûne, la variante avec [ø] fermé du locuteur-modèle est préférée par une grande majorité, hautement significative ( $p<0,001)$, de 62,50\% (60/96) des informateurs et est suivie par ce même [ø] mais chez le locuteur « ordinaire » $(27,08 \%, 26 / 96)$ (cf. figure 105).

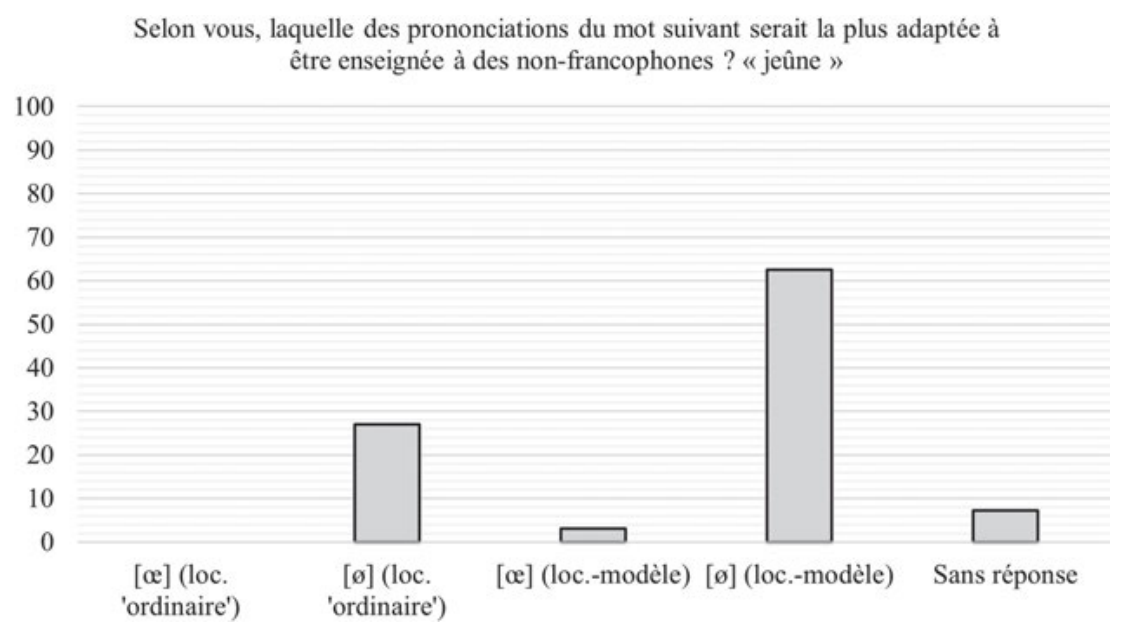

Figure 105 : Voyelle privilégiée (en \%) dans le mot <jeûne> pour les cours de français langue étrangère (FLE) $(n=96)$ - Mots isolés ( $p<0,001$, coef. $=4,11$, e.s. $=0,60, t=6,88)$.

Le résultat obtenu dans le cas de la deuxième question relative au mot isolé se confirme donc ici : la variante avec [ø] fermé du locuteur-modèle étant préférée dans un contexte de comparaison directe et d'isolation du trait dans le mot jeûne, la préférence des informateurs semble être au maintien de l'opposition /ø/ : /œ/.

La même question posée sur la base du stimulus syntagmatique engendre des résultats certes globalement similaires - avec une préférence significativement majoritaire pour le /ø/ fermé du locuteur-modèle $(87,50 \%, 84 / 96)$-, mais, ici aussi, différents quant au stimulus figurant en seconde position : dans ce cas-ci, il s’agit en effet du [œ] ouvert de ce même locuteur-modèle (12,50\%, 12/96) et non du [ø] fermé du locuteur « ordinaire » (cf. figure 106). 
Selon vous, laquelle des deux prononciations du groupe de mots suivant serait la plus adaptée à être enseignée à des non-francophones ?

«Quelques fanatiques auraient même entamé un jeûne prolongé dans l'église de Saint Martinville »

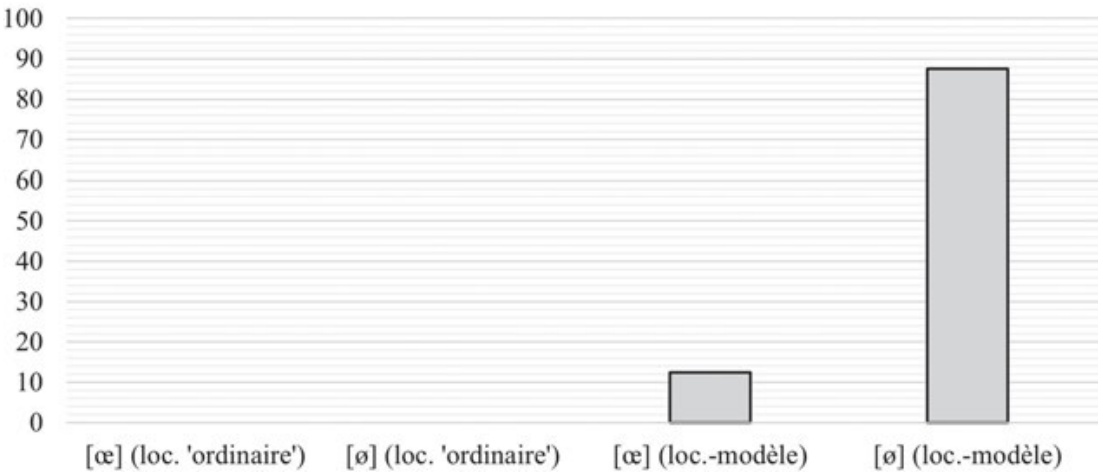

Figure 106 : Voyelle privilégiée (en \%) dans le mot <jeûne> pour les cours de français langue étrangère (FLE) $(\mathrm{n}=96)$ - Texte PFC $(p<0,001$, coef. $=0,95$, e.s. $=0,09, t=11,13)$.

Ainsi, en plus du principal résultat qui, dans l'optique de la présente étude, montre que la variante avec [ø] fermé du locuteur-modèle est largement préférée, ces données suggèrent également que le degré de saillance de ce trait est relativement faible, les perceptions semblant, comme dans le cas de la deuxième question, se focaliser davantage sur le type de locuteur, le locuteur-modèle étant préféré au locuteur " ordinaire ». Relevons par ailleurs un léger effet significatif du niveau d'éducation des informateurs ( $p<0,05$, coef. $=0,08$, e.s. $=0,04, t=2,13$ ), ceux qui n'ont pas le baccalauréat ayant à nouveau tendance à choisir légèrement plus souvent la variante ouverte [œ] du locuteur-modèle $(18,75 \%, 9 / 48)$ que les informateurs titulaires du baccalauréat $(6,25 \%, 3 / 48)$. Mais ici aussi, l'effet reste très marginal, étant donné que ces locuteurs non diplômés préfèrent également largement plus souvent la variante fermée [ø] de ce même locuteur-modèle (81,25\%, 39/48).

En conclusion, trois principales observations peuvent être faites par rapport à ces résultats : premièrement - et il s'agit du résultat essentiel dans l'optique de la présente étude -, les informateurs ont fortement tendance à privilégier la variante avec [ø] fermé dans le mot jeûne, ce qui plaiderait en faveur du maintien de l'opposition dans les perceptions des informateurs, et confirme les résultats des productions des locuteurs-modèles (cf. 5.1.1.3).

Mentionnons cependant que le problème de la différenciation entre perceptions et représentations (cf. 3.1) se pose également ici : il est en effet possible que les résultats touchant aux mots isolés relèvent plus de représentations que de perceptions, étant donné que le trait devant être évalué y est très explicite, alors qu'il est plus implicite dans les syntagmes. En raison de ce problème méthodologique, 
de futures études devront donc montrer dans des contextes linguistiques plus implicites et naturels dans quelle mesure ces résultats peuvent être confirmés.

Deuxièmement, les résultats des questions 2 et 3 relatives au stimulus syntagmatique présentent deux points intéressants : tout d'abord, l'opposition ne semble pas particulièrement saillante dans les perceptions des informateurs, puisque dans un contexte où elle n'est pas isolée, elle tend à ne pas être perçue par les informateurs. Dans un tel cas, comme déjà observé dans le cas de l'opposition /e/ : / $\varepsilon$ / (cf. 6.1.2), il semble en effet que ce soit la perception globale de la lecture du stimulus qui prenne le dessus dans les perceptions. Et ce faisant, les stimuli des locuteurs-modèles, peut-être identifiés comme des présentateurs, sont systématiquement mieux évalués que les stimuli des locuteurs parisiens « ordinaires ».

Troisièmement, il existe également un effet relativement régulier du niveau d'éducation sur les résultats : l'utilisation de la variante ouverte [œ] semble en effet légèrement mieux acceptée par les informateurs les moins diplômés. Ainsi, au niveau des perceptions, la neutralisation de l'opposition dans la paire minimale jeune jeûne en faveur du [œ] ouvert semble être légèrement plus avancée dans ce groupe sociodémographique, même s'il faut également remarquer que dans ces perceptions, les indices d'une telle neutralisation restent très faibles.

\subsubsection{Opposition / $\tilde{e} /: / \tilde{\varepsilon} /$}

Le quatrième et dernier trait soumis aux évaluations perceptives touche à l'opposition des voyelles nasales /õ̃/ : / $\tilde{\varepsilon} /$. Rappelons que l'analyse des productions a montré que l'opposition peut être considérée comme largement neutralisée en

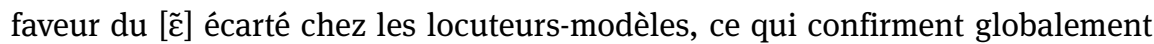
les résultats de Hansen (2001 ; 2012 ; 2017), Pustka (2008) et Lyche/Østby (2009). Les résultats ont par ailleurs montré la plus forte résistance de l'adjectif brun au processus de neutralisation. Ces résultats seront confrontés ci-dessous aux données de perception.

\section{Question 1 : évaluation (sans contexte explicite)}

Les résultats de la question d'évaluation sur une échelle (0\%-100\%) montrent que les variantes arrondies [õ]] sont globalement préférées aux variantes écartées

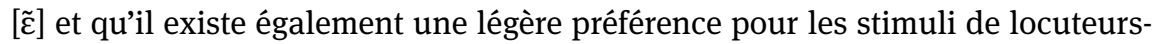
modèles. En effet, la variante avec [œẽ] arrondi du locuteur « ordinaire » obtient un score de 76,44\% (écart-type : 16,04\%), contre un score de 59,99\% (écart-type :

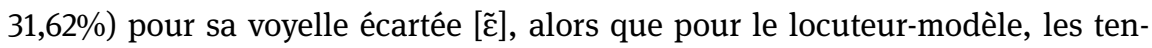


dances sont similaires, mais avec des scores légèrement plus élevés ([øõ] : 83,56\%, écart-type : 16,70\%; [ $\tilde{\varepsilon}]$ : 65,94\%, écart-type : 25,62\%). L'analyse de variance (ANOVA) indique, pour sa part, que seule la différence entre les évaluations du

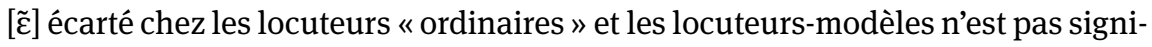
ficative, toutes les autres l'étant à $p<0,001$ ou $p<0,01$ (cf. figure 107).

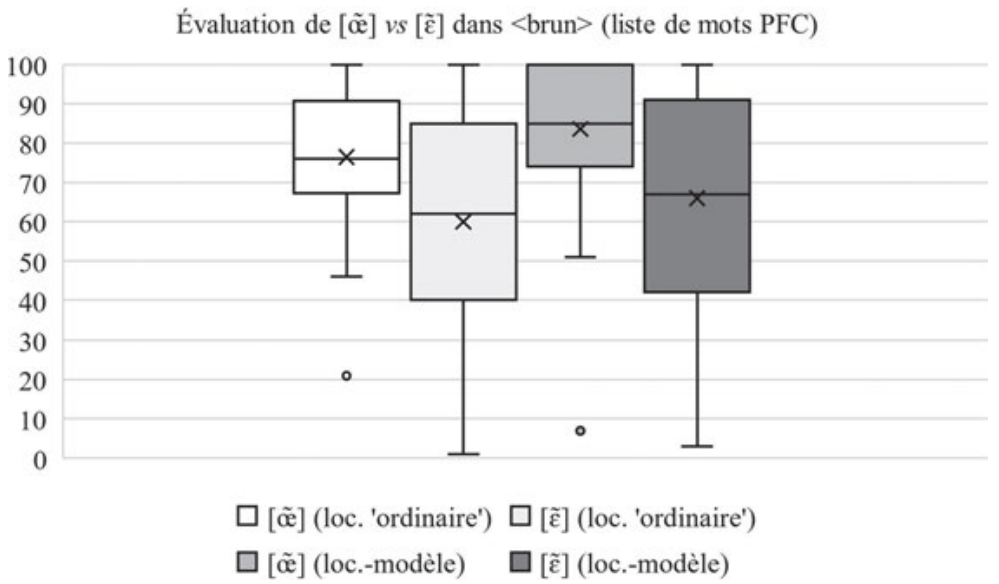

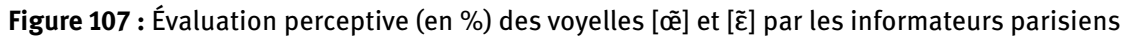

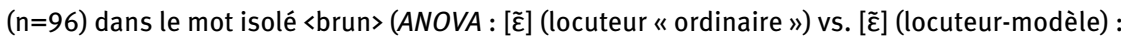
$p>0,05$; [ø̃] (locuteur « ordinaire ») vs. [0̃] (locuteur-modèle) : $p<0,01$; autres différences de moyennes : $p<0,001)$.

Notons par ailleurs que le modèle révèle à nouveau un effet - à différents degrés de signification - du niveau d'éducation sur les résultats des trois premières variantes ([ø̃̃] locuteur « ordinaire »: $F=11,83, p<0,001 ;[\tilde{\varepsilon}]$ locuteur « ordinaire » : $F=7,34, p<0,01$; [œе̃] locuteur-modèle : $F=6,79, p<0,05)$. Variantes de référence, les variantes arrondies [õ] sont en effet légèrement mieux évaluées par les informateurs détenant le baccalauréat que par les informateurs sans bacca-

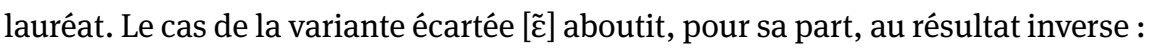
elle est en effet légèrement moins bien évaluée par les titulaires du baccalauréat. L'utilisation de la variante écartée $[\tilde{\varepsilon}]$ pour la graphie $<$ un $>$ semble donc mieux tolérée dans les perceptions des informateurs moins diplômés.

Posée par rapport au déterminant un dans le syntagme d'un autre côté, cette même question entraîne des réponses fortement ambiguës : les évaluations des quatre variantes s'avèrent en effet très similaires ([õ̃] locuteur

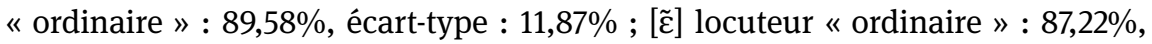
écart-type : 16,24\% ; [œẽ] locuteur-modèle : 86,72\%, écart-type : 11,09\% ; [ [ ] 
locuteur-modèle : $86,96 \%$, écart-type : $11,58 \%$ ) et l'analyse de variance ne permet de déceler aucune différence significative entre elles ( $p>0,05)$ (cf. figure 108).

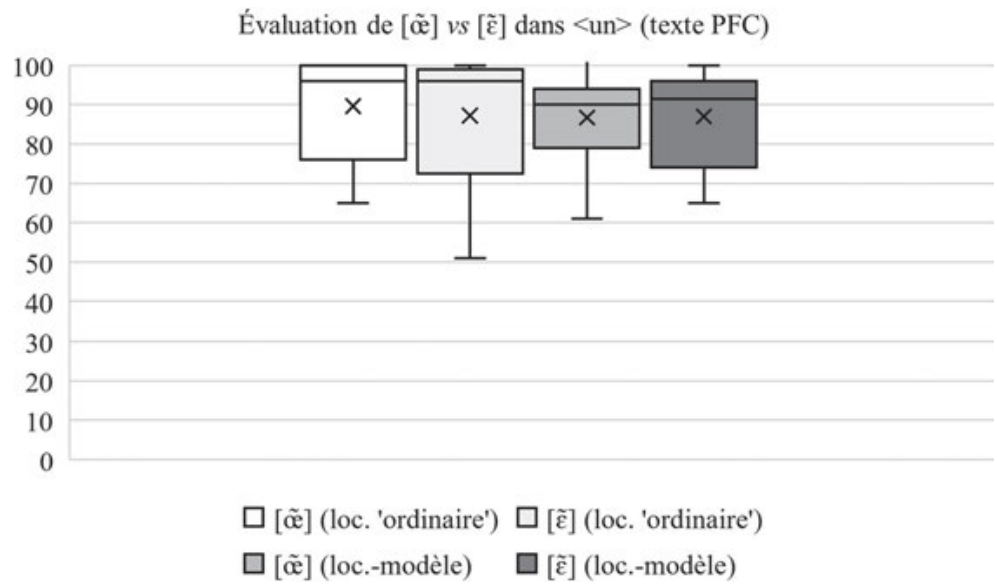

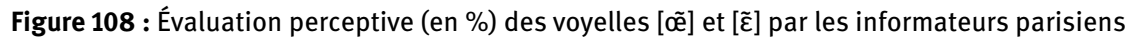
$(n=96)$ dans le mot <un> tiré du texte PFC (ANOVA : différences entre les différentes moyennes non significatives : $p>0,05$ ).

Ainsi, alors que dans le contexte du mot isolé brun, la tendance semble être de privilégier la variante arrondie [œẽ], la différence s'estompe perceptivement dans un contexte linguistique utilisant un stimulus syntagmatique d'une certaine longueur et/ou le déterminant un. Deux facteurs semblent jouer un rôle ici : il est en effet probable que le degré de saillance de l'opposition soit relativement faible dans les perceptions des Parisiens, ce qui la rendrait difficilement perceptible dans un contexte linguistique plus long, comme dans le cas présent. De plus, il existe sans doute également un effet lexical, l'analyse des productions ayant déjà montré auparavant que brun pourrait présenter une plus grande résistance que le déterminant un au processus de neutralisation de l'opposition aboutissant à

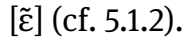

\section{Question 2 : prononciation idéale des présentateurs de journaux télévisés}

Les résultats de la question sur la prononciation-cible pour les présentateurs de journaux télévisés confirment largement les deux tendances observées dans le cadre de la première question : d'une part, les informateurs préfèrent globalement la voyelle arrondie [œ̃̃], d'autre part, ils la préfèrent dans l'usage du locuteurmodèle ([œẽ] locuteur-modèle : 69,97\%, 67/96; [œẽ] locuteur « ordinaire » : 14,58\%, 14/96), cette différence étant hautement significative ( $p<0,001)$ (cf. figure 109). 
Selon vous, comment un présentateur de télévision parisien devrait-il prononcer le mot suivant? « brun »

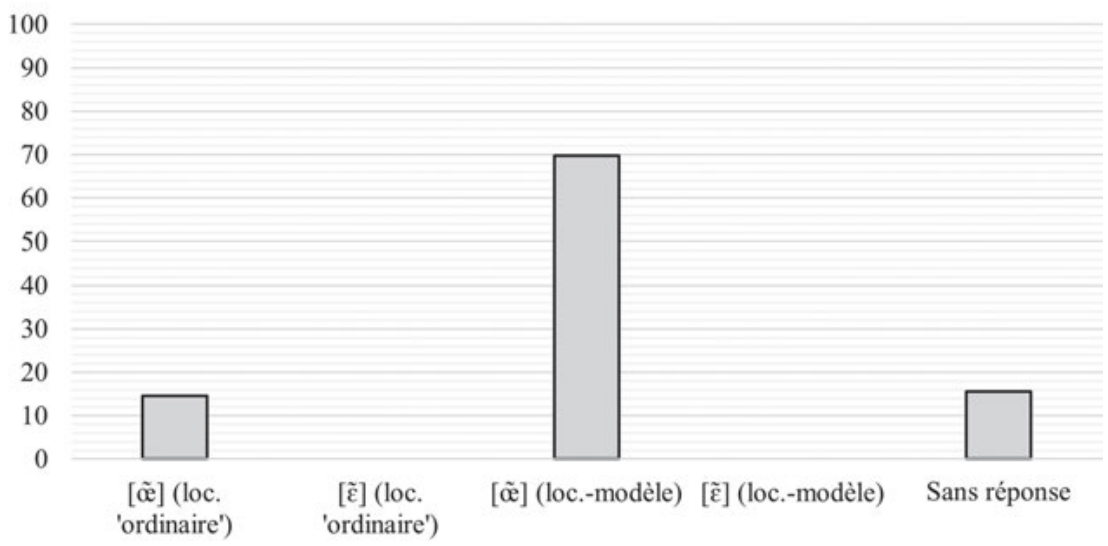

Figure 109 : Voyelle privilégiée (en \%) dans le mot <brun> pour les présentations de journaux télévisés $(\mathrm{n}=96)$ - Mots isolés $(p<0,001$, coef. $=3,86$, e.s. $=0,30, t=12,95)$.

En ce qui concerne le syntagme d'un autre côté, les résultats sont quelque peu différents. Même si une certaine préférence pour les variantes contenant un [õ̃] arrondi peut être observée ([õ̃] locuteur-modèle : 42,71\%, 41/96 ; [õ̃] locuteur « ordinaire » :29,17\%, 28/96), l'ambiguïté dans le choix de la variante est largement plus grande que dans le cas du mot isolé brun, les deux variantes contenant un [च̃] écarté étant également privilégiées par un certain pourcentage d'informateurs

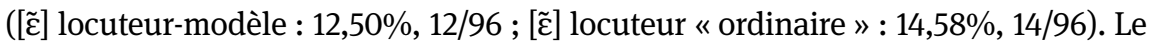
modèle de régression montre d'ailleurs que les différences entre les quatre variantes s'avèrent, certes, significatives, mais à un niveau plus faible $(p<0,05)$ (cf. figure 110).

Ainsi, bien que la préférence d'ensemble pour de la variante arrondie [õ̃], particulièrement chez les locuteurs-modèles, se vérifie, les présents résultats tendent également à confirmer les deux hypothèses formulées plus haut (cf. supra) : d'une part, le degré de saillance de l'opposition /õ̃/: / $/ \tilde{\varepsilon} /$ pourrait s'avérer relativement faible. D'autre part, il est probable que l'effet lexical joue un rôle dans les évaluations, le [õ̃] résistant davantage au processus de neutralisation dans l'adjectif brun que dans le déterminant un, très fréquent.

\section{Question 3 : prononciation à enseigner dans les cours de français langue étrangère (FLE)}

Les résultats obtenus dans la troisième question, qui touche à la prononciationcible pour le FLE, ne se différencient pas notablement de ceux de la question sur la prononciation idéale de locuteurs-modèles : dans le cas du mot isolé brun tout 
Selon vous, comment un présentateur de télévision parisien devrait-il prononcer le groupe de mots suivant? «D'un autre côté »

$$
\begin{array}{r}
100 \\
90 \\
80 \\
70 \\
60 \\
50 \\
40 \\
30 \\
20 \\
10
\end{array}
$$$$
0
$$

[थ̃] (loc. 'ordinaire')

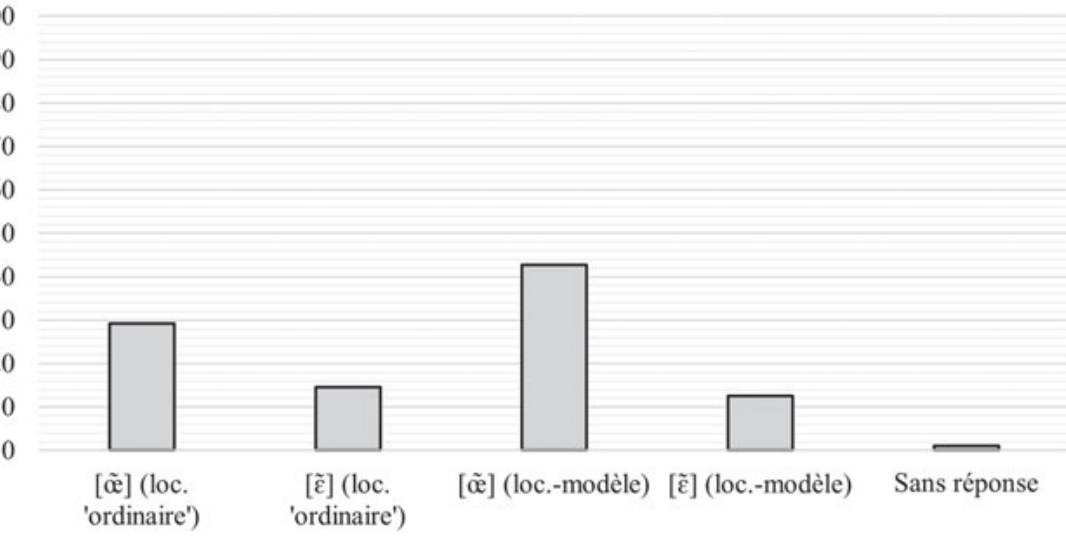

Figure 110 : Voyelle privilégiée (en \%) dans le mot <un> pour les présentations de journaux télévisés $(\mathrm{n}=96)$ - Texte PFC $(p<0,05$, coef. $=0,92$, e.s. $=0,38, t=2,43)$.

d'abord, la tendance est de préférer largement le [õ] arrondi chez le locuteurmodèle $(63,54 \%, 61 / 96)$, dont le taux est significativement plus élevé que celui de toutes autres variantes, et ce, à $p<0,001$ (cf. figure 111).

Selon vous, laquelle des deux prononciations du mot suivant serait la plus adaptée à être enseignée à des non-francophones ? «brun »

$$
\begin{array}{r}
100 \\
90 \\
80 \\
70 \\
60 \\
50 \\
40 \\
30 \\
20 \\
10 \\
0
\end{array}
$$

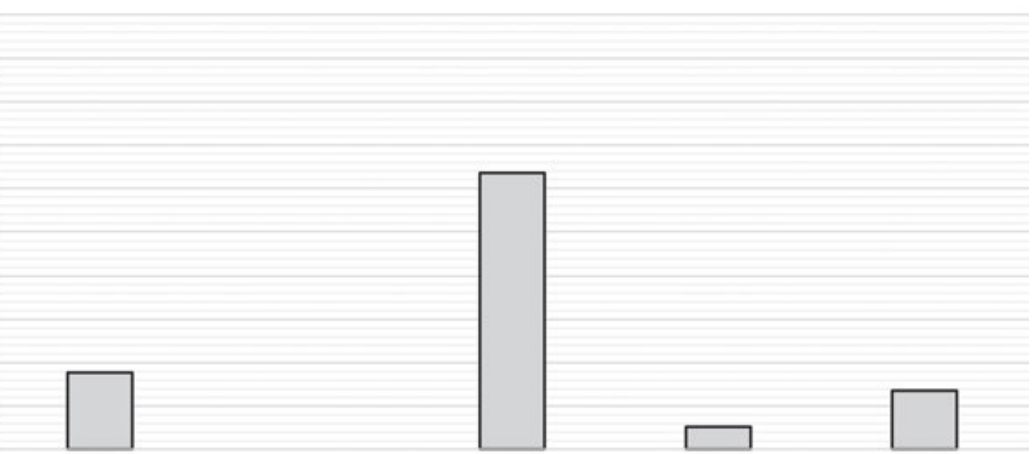

[थ̃] (loc. 'ordinaire')

[ह̃] (loc. 'ordinaire')

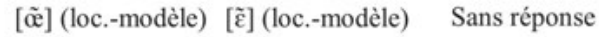

Figure 111 : Voyelle privilégiée (en \%) dans le mot <brun> pour les cours de français langue étrangère (FLE) $(n=96)$ - Mots isolés ( $p<0,001$, coef. $=3,45$, e.s. $=0,66, t=5,22)$. 
Tout comme dans le cas de la deuxième question, les évaluations du syntagme d'un autre côté montrent, au contraire, une ambiguïté considérable. La variante arrondie [œẽ] du locuteur-modèle est, certes, légèrement préférée par les informateurs $(36,46 \%, 35 / 96)$, mais son taux ne se différencie pas significativement de celui des autres variantes selon le modèle de régression $(p>0,05)$. Relevons également qu'au contraire du cas du mot isolé brun, le stimulus arrivant en deuxième position selon le taux de réponses n'est pas celui du locuteur « ordinaire » présentant la variante arrondie [œẽ], mais celui du locuteur-modèle avec la

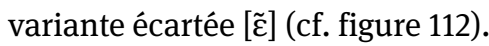

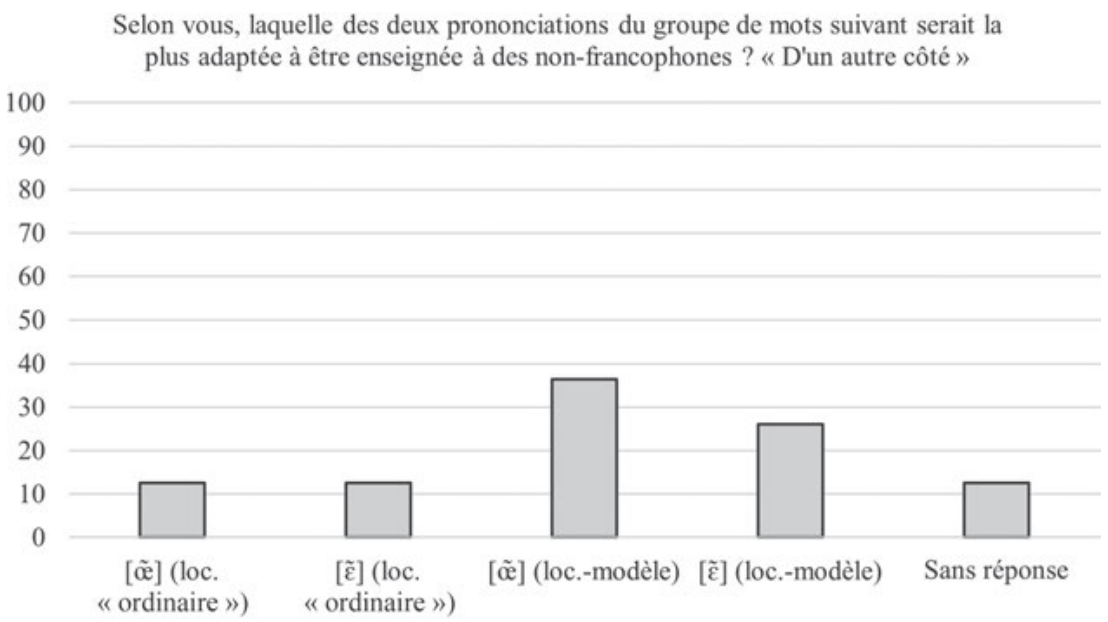

Figure 112 : Voyelle privilégiée (en \%) dans le mot <un> pour les cours de français langue étrangère (FLE) $(\mathrm{n}=96)$ - Texte PFC ( $p>0,05$, coef. $=0,73$, e.s. $=0,37, t=1,99)$.

Dans le cas du syntagme soumis aux évaluations, il existe apparemment, ici aussi, une tendance des informateurs à préférer, d'une part, le [õ̃] arrondi dans le

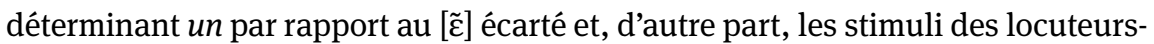
modèles par rapport à ceux des locuteurs « ordinaires ». Mais du fait de la grande ambiguïté de ces réponses, le faible degré de saillance de l'opposition dans les perceptions des informateurs semble également se confirmer.

En conclusion, les résultats touchant à l'opposition /œẽ/ : / $\tilde{\varepsilon} /$ ont permis de révéler trois éléments fondamentaux dans l'optique de la présente étude : tout d'abord, contrairement à ce que montrait l'analyse des productions (cf. 5.1.2), le [õ̃] arrondi est majoritairement privilégié dans les perceptions des informateurs parisiens. Pourtant, les différences de résultats entre le mot isolé brun et le déterminant un, testé dans le cadre du syntagme d'un autre côté, suggèrent 
également deux autres observations : d'une part, il semblerait que les informateurs attendent plus le [œẽ] arrondi dans l'adjectif brun que dans le déterminant un, ce qui confirmerait perceptivement les résultats des analyses de production indiquant une plus grande résistance de l'adjectif brun au processus de neutralisation. D’autre part, il est probable que la saillance de l'opposition soit très faible dans la perception des informateurs, de sorte qu'elle est peu ou pas perçue dans des stimuli plus longs.

Enfin, mentionnons également qu'étant donnée la différence entre les résultats du mot isolé brun et du syntagme d'un autre côté, il est à nouveau vraisemblable que les réponses touchant au mot isolé soient davantage le fruit de représentations que de perceptions (cf. 3.1). De futures études devront donc, ici aussi, montrer dans quelle mesure ces résultats peuvent être confirmés lorsque des stimuli plus implicites et naturels sont utilisés.

\subsubsection{Discussion}

Dans ce premier sous-chapitre, quatre traits ont été soumis aux évaluations perceptives de 96 informateurs parisiens sélectionnés selon un échantillonnage par quotas sur la base de quatre critères sociodémographiques (origine, sexe, âge et niveau d'éducation des informateurs) (cf. 3.3.2). Il s'agit de quatre traits déjà analysés dans les productions de locuteurs-modèles parisiens au chapitre précédent (cf. 5.1). Le tableau 74 présente un aperçu des principaux résultats des tests de perception et les compare aux résultats de ces analyses de production, afin de permettre l'estimation la plus fiable possible du statut de ces traits dans la « norme de prononciation parisienne».

Notons que l'aperçu proposé dans le tableau 74 révèle tout d'abord de grandes différences entre les résultats des évaluations perceptives et ceux des analyses de production. En effet, alors que les productions indiquaient pour deux des quatre oppositions une neutralisation majoritaire (cf. tableau 74 : traits 1, 4) et pour les deux autres, à tout le moins, une tendance à la neutralisation (cf. tableau 74 : traits 2, 3), les perceptions montrent que les informateurs continuent à privilégier majoritairement le maintien de ces quatre oppositions. Une explication régulièrement mentionnée dans le chapitre pourrait apporter certains éléments de réponse par rapport à cet écart : les différences frappantes observées pour les quatre traits entre les résultats des mots isolés et ceux des syntagmes suggèrent que les réponses touchant aux mots isolés pourraient être davantage le fruit de représentations que de perceptions (cf. 3.1 pour la différence entre ces deux concepts). La présentation sous forme de paires de mots rend en effet l'opposition si manifeste qu'il est probable que les auditeurs aient plutôt fait appel à leur 
Tableau 74 : Comparaison des résultats touchant aux productions et perceptions pour les quatre traits étudiés dans le cas de Paris.

\begin{tabular}{|c|c|c|c|c|}
\hline $\begin{array}{l}\text { Traits de prononciation } \\
\text { parisiens }\end{array}$ & Contextes testés & Productions & Perceptions & Commentaires \\
\hline 1. Opposition /a/:/a/ & pâte: : [pat] [pat] & $\mid a /$ & $|a|:|a|$ & \multirow{4}{*}{$\begin{array}{l}\text { Perception : } \\
\text { faible degré } \\
\text { de saillance } \\
\text { des quatre } \\
\text { oppositions }\end{array}$} \\
\hline 2. Opposition /ع/:/e/ & 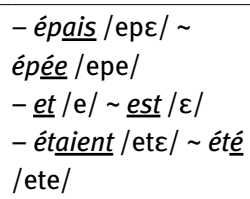 & $\begin{array}{l}-/ \varepsilon /: / \mathrm{e} / \\
-/ \mathrm{e} / \\
-/ \mathrm{e} /\end{array}$ & $\begin{array}{l}-/ \varepsilon /: / \mathrm{e} / \\
-/ \varepsilon /: / \mathrm{e} / \\
\text { (tendance }: / \mathrm{e} / \text { ) } \\
-/ \varepsilon /: / \mathrm{e} /\end{array}$ & \\
\hline 3. Opposition /œ/: /ø/ & jeûne : [3œn] [3øn] & $\begin{array}{l}\text { /œ/:/ø/ } \\
\text { (tendance : } \\
/ œ / \text { ) }\end{array}$ & $/ œ /: / \varnothing /$ & \\
\hline 4. Opposition / $\tilde{e} /: / \tilde{\varepsilon} /$ & 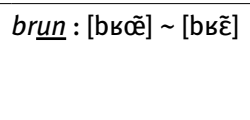 & $\mid \tilde{\varepsilon} /$ & $\begin{array}{l}\text { / œ̃ } /: / \tilde{\varepsilon} / \\
\text { (tendance }: / \tilde{\varepsilon} / \\
\text { dans } \text { un) }\end{array}$ & \\
\hline
\end{tabular}

connaissance explicite de ces oppositions dans leurs représentations qu'à leurs perceptions réelles des stimuli. Ceci est d'autant plus vraisemblable que le type de tâche - les stimuli entendus devaient être évalués par rapport au mot apparaissant à l'écran sous sa forme orthographique (cf. 3.5.2) - pourrait avoir favorisé la référence aux représentations, de nature plus explicite que les perceptions.

Parallèlement à cette observation, mentionnons par ailleurs que la longueur des stimuli syntagmatiques semble avoir perturbé les informateurs dans leurs évaluations, en ce sens que le contexte linguistique (plus ou moins) long a sans doute régulièrement empêché la bonne perception des traits à évaluer. Par conséquent, ces mêmes stimuli syntagmatiques semblent donc également avoir mis en lumière le degré de saillance relativement faible de ces quatre oppositions dans les perceptions des informateurs parisiens.

Il est ainsi difficile de donner des conclusions tranchées par rapport à ces quatre traits, mais une réduction schématique de la complexité des résultats montre tout de même certaines tendances. Premièrement, le cas de l'opposition /a/ : /a/ présente une forte ambiguïté, l'opposition n'étant pas maintenue dans les productions, tout en étant privilégiée dans les perceptions/représentations Pour ce trait, une conclusion claire est donc malaisée. Deuxièmement, le cas de l’opposition $/ \varepsilon /: / e /$ est très ambigu, mais dépend de la paire minimale prise en compte : dans la paire de lexèmes peu fréquents épais /epe/ épée /epe/, l’opposition semble être maintenue dans la norme de prononciation. Dans la paire été /ete/ étaient /ete/, les productions et perceptions engendrent des résultats 
contraires, rendant les résultats difficiles à interpréter. Dans la paire de lexèmes très fréquents et /e/ est / $\varepsilon /$, malgré des résultats pas totalement convergents entre productions et perceptions, la tendance est relativement clairement à la neutralisation de l'opposition en faveur du /e/ fermé. Troisièmement, l'opposition /ø/: /œ/ est maintenue, à tout le moins, dans la paire minimale jeune [3œn] jeûne [3øn], bien qu'un début de neutralisation en faveur du [œ] ouvert soit observable dans les productions. Finalement, l'opposition /œẽ/ : / $\tilde{\varepsilon} /$ présente une forte ambiguïté étant donnés les résultats contradictoires des productions et des perceptions/représentations. Cependant, comme la tendance est à la prononciation $/ \tilde{\varepsilon} / \mathrm{du}<\mathrm{un}>$ graphique dans le déterminant très fréquent $u n$, y compris dans les perceptions/représentations, l'opposition connaît manifestement une neutralisation croissante.

Soulignons par ailleurs que le niveau d'éducation des informateurs s'est avéré être la seule variable sociodémographique à présenter de manière régulière des effets significatifs sur les résultats. En effet, dans le cas des quatre oppositions, les informateurs ayant un niveau d'éducation plus élevé ont présenté une tendance, plus ou moins prononcée selon la question, en faveur de la variante de référence ([a] dans pâate, cf. 6.1.1 ; [ع] dans épais, est, étaient, $\mathrm{cf}$. 6.1.2 ; [ø] dans jêune, cf. 6.1.3 ; [ø̃̃] dans brun, cf. 6.1.4). Ils semblent par ailleurs plus enclins à percevoir l'opposition dans des contextes linguistiques plus longs (contextes syntagmatiques). Les quatre oppositions paraissent ainsi légèrement plus saillantes et plus souvent maintenues chez les informateurs plus diplômés, alors que ceux dont le niveau d'éducation est moins élevé ont davantage tendance à ne pas percevoir le trait dans ces contextes plus longs et/ou à tolérer les variantes traditionnellement exclues de la norme de référence (c'est-à-dire

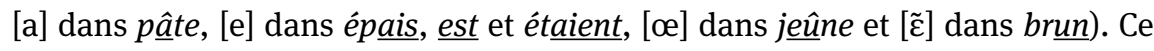
résultat pourrait s'expliquer par deux facteurs : il se peut que les informateurs ayant un niveau d'éducation supérieur connaissent mieux les représentations de la norme dans leur communauté linguistique, et il est également possible qu'ils soient linguistiquement plus conservateurs que ceux dont le niveau d'éducation est moins élevé.

En définitive, malgré ces quelques différences par rapport à la norme de référence, il est observable que la « norme parisienne » testée ici et la traditionnelle norme de prononciation de référence restent - du moins pour ce qui est de ces quatre traits - très proches et difficilement dissociables l'une de l'autre. Ainsi, alors qu'il existe bien, à tout le moins, trois accents micro-diatopiques (et sociaux) parisiens dans les auto-représentations des Parisiens (accents « du 16 ${ }^{\text {ème }}$ », «banlieusard » ou « faubourien »; cf. 4.4.1.2), il est difficile de suggérer l'existence d'une « norme de prononciation parisienne » parallèlement à la norme de prononciation « de référence » dans ces mêmes auto-représentations. Cette obser- 
vation différencie donc considérablement le cas de Paris de celui du Québec (cf. 6.3.9) et, dans une moindre mesure, de celui de la Suisse romande (cf. 6.2.6).

Mentionnons finalement que plusieurs réserves auront pu être faites par rapport à la méthodologie du test de perception. Étant donné que ces réserves sont également pertinentes pour les tests de perception effectués en Suisse romande et au Québec, elles ont été rassemblées dans un sous-chapitre prévu à cet effet (cf. 6.4).

\subsection{Suisse romande}

Les tests de perception effectués en Suisse romande portent sur les cinq traits vocaliques analysés dans le chapitre sur la production (cf. 5.2), tirés des études de Métral (1977), Andreassen/Lyche (2009), Andreassen/Maître/Racine (2010), Pooley (2012) et Racine (2016) : l’opposition /a/ : /a:/ en syllabe finale fermée (cf. 6.2.1), l'opposition /e/ : /e:/ en syllabe finale fermée (cf. 6.2.2), l'opposition /e/ : $/ \varepsilon /$ en syllabe finale ouverte permettant de distinguer certains temps et modes (cf. 6.2.3), la préférence de la diérèse à la synérèse (cf. 6.2.4) et l'opposition /œẽ/ : / $\tilde{\varepsilon} /$ (cf. 6.2.5).

\subsubsection{Opposition /a/ : /a: en syllabe finale fermée}

Le premier trait évalué perceptivement concerne l'opposition de durée et de timbre /a/ : /a:/ en syllabe finale fermée. Dans l'analyse des productions, cette opposition a été mise en évidence chez une majorité des présentateurs dans la paire minimale patte p pâte, même si la tendance actuelle dans ces mêmes productions est à la neutralisation de l'opposition de timbre (/a/ : /a:/), voire de durée (/a/ antérieur bref unique) (cf. 5.2.1.1).

\section{Question 1 : évaluation (sans contexte explicite)}

Les résultats touchant à l'évaluation perceptive effectuée sur la base du mot pâte et d'une échelle (0\%-100\%) sans mention explicite d'un contexte montrent tout d'abord une large préférence pour la variante postérieure et allongée [a:] (évaluation moyenne : 86,25\%, écart-type : 22,58\%) par rapport au [a] antérieur et bref (évaluation moyenne : 41,02\%, écart-type : 27,81\%). L’analyse de variance indique en effet une différence hautement significative à $p<0,001$ entre les évaluations des deux voyelles (cf. figure 113). 


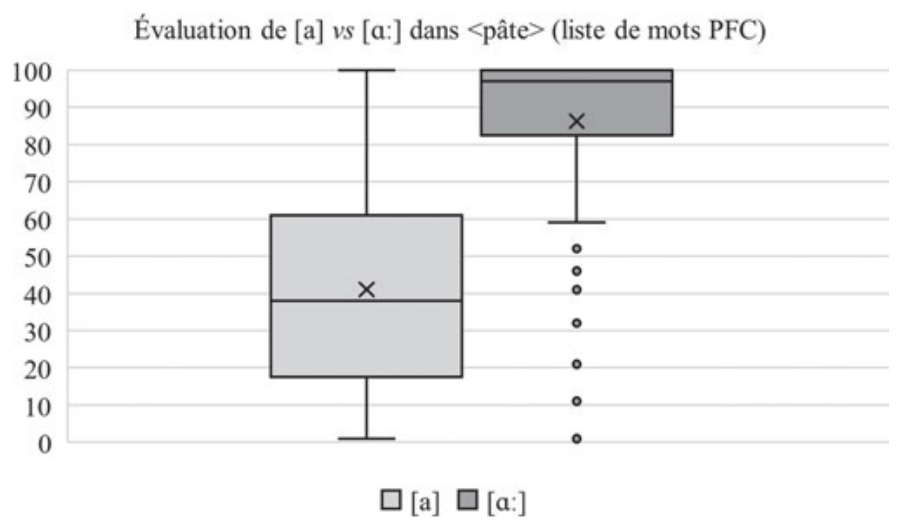

Figure 113 : Évaluation perceptive (en \%) des voyelles [a] et [a:] par les informateurs suisses romands $(\mathrm{n}=96)$ dans le mot isolé <pâte> (ANOVA : $p<0,001)$.

En ce qui concerne l'influence des variables sociodémographiques, l'analyse de variance ne révèle par ailleurs qu'un seul effet significatif parmi les quatre facteurs pris en compte dans le modèle (sexe, âge, origine, niveau d'éducation). En effet, elle indique une légère influence positive de l'âge sur les évaluations du [a:] $(F=3,56, p<0,05)$ : plus les informateurs sont âgés, plus ils auront tendance à évaluer positivement ce [a:]. Ce résultat va donc dans le sens des observations faites dans l'analyse des productions : le [a:] semble être plus souvent produit et mieux évalué par les Suisses romands d'un âge plus avancé.

Quant à l'évaluation des deux variantes de ce même mot pâte dans le syntagme et plus récemment son usine de pâtes italiennes, elle montre des résultats très différents : les deux variantes sont en effet évaluées de manière très similaire, avec un score de 71,92\% (écart-type : 23,69\%) pour le [a], contre un score de 73,06\% (écart-type : $25,56 \%)$ pour le [a:], la différence n’étant pas significative $(p>0,05)$ (cf. figure 114).

Ces résultats étant très similaires, il n'est pas étonnant qu'aucun des facteurs de l'analyse de variance ne présente d'effet significatif $(p>0,05)$. La différence entre les résultats respectifs du mot pâte, isolé ou dans le contexte du syntagme et plus récemment son usine de pâtes italiennes, est probablement due à la longueur considérable du syntagme testé et au fait que, contrairement aux questions 2 et 3, l'évaluation de chaque variante a été effectuée dans une question distincte, c'est-à-dire sans comparaison directe des deux variantes : les informateurs ont donc pu avoir des difficultés à reconnaître le trait à évaluer. Ainsi, si l'interprétation globale des résultats de cette question va bien en direction d'une préférence pour la variante postérieure et allongée [a: dans le mot pâte, le syntagme semble indiquer un degré de saillance relativement faible de l'opposition, ce qui la rend moins facilement perceptible dans des contextes linguistiques plus longs. 
Évaluation de [a] $v s[\mathrm{a}:]$ dans <pâte $>$ (texte PFC)

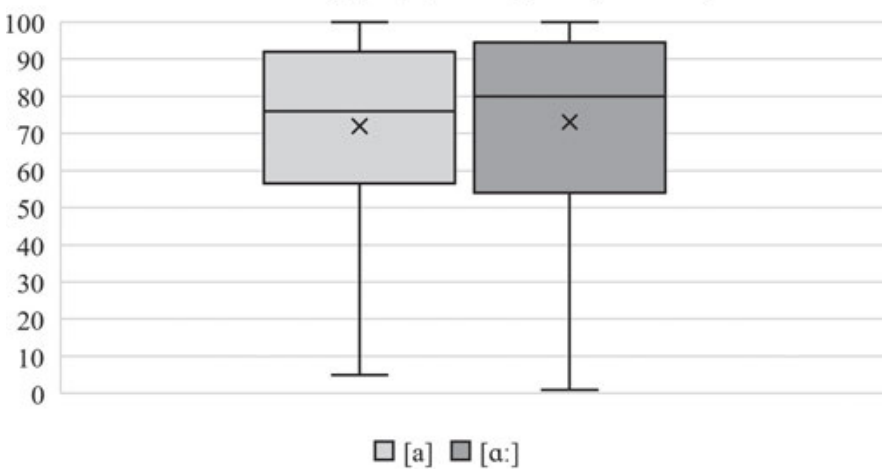

Figure 114 : Évaluation perceptive (en \%) des voyelles [a] et [a:] par les informateurs suisses romands $(\mathrm{n}=96)$ dans le mot <pâte $>$ tiré du texte PFC $($ ANOVA : $p>0,05)$.

\section{Question 2 : prononciation idéale des présentateurs de journaux télévisés}

En ce qui concerne l'évaluation perceptive de ces mêmes variantes dans le contexte de la prononciation perçue comme idéale pour un présentateur de télévision, les résultats montrent pour le mot isolé <pâte> la même tendance à privilégier le [a:] postérieur et allongé : 90,63\% (87/96) des informateurs choisissent la variante [a:], contre seulement $8,33 \%$ (8/96) pour la variante [a], la différence étant hautement significative à $p<0,001$ (cf. figure 115).

Selon vous, comment un présentateur de télévision suisse devrait-il prononcer le mot suivant ? «pâte »

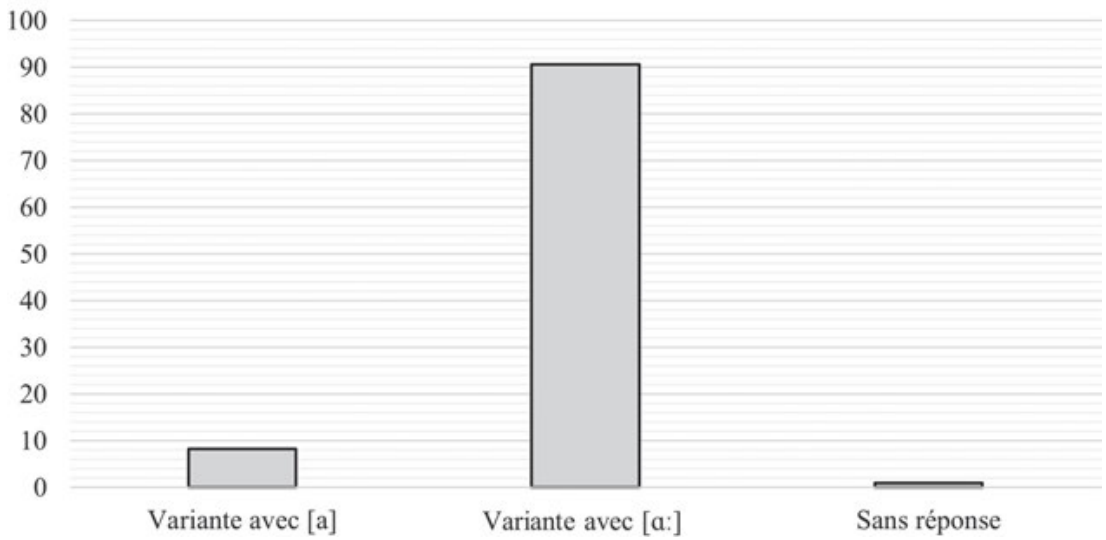

Figure 115 : Voyelle privilégiée (en \%) dans le mot <pâte> pour les présentations de journaux télévisés $(\mathrm{n}=96)$ - Mots isolés $(p<0,001$, coef. $=2,11$, e.s. $=0,14, t=15,20)$. 
Cette même question, posée sur la base du syntagme et plus récemment son usine de pâtes italiennes, obtient des résultats similaires, bien que moins univoques : la variante [a:] a été choisie par 52,08\% (50/96) des informateurs, contre $36,46 \%$ (35/96) des informateurs privilégiant la variante [a], cette différence étant également significative à $p<0,001$ (cf. figure 116).

Selon vous, comment un présentateur de télévision suisse devrait-il prononcer le groupe de mots suivant ?

« Et plus récemment son usine de pâtes italiennes »

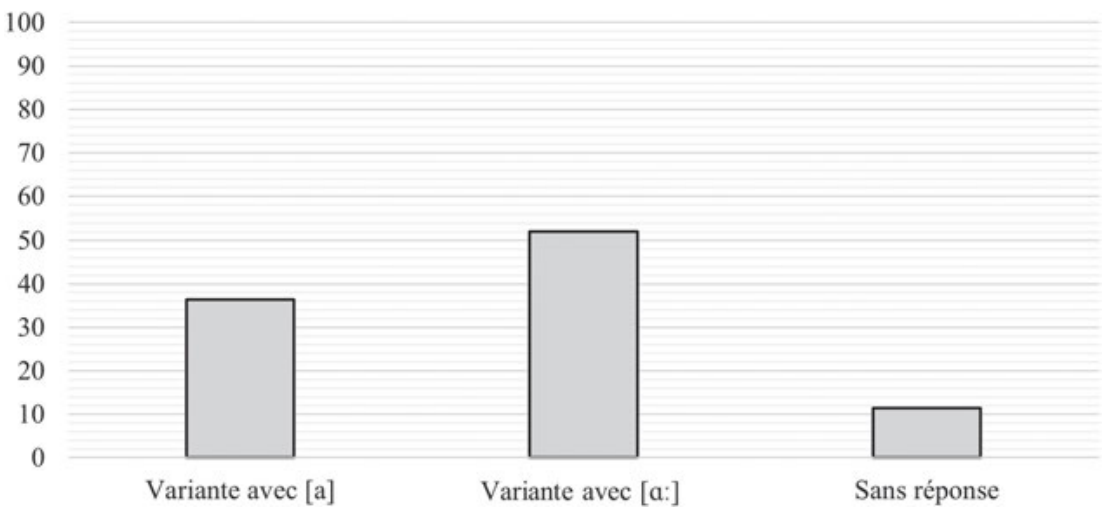

Figure 116 : Voyelle privilégiée (en \%) dans le mot <pâte> pour les présentations de journaux télévisés $(\mathrm{n}=96)$ - Texte PFC $(p<0,001$, coef. $=1,68$, e.s. $=0,31, t=5,43)$.

Ainsi, malgré des tendances moins claires dans le cas du syntagme tiré du texte PFC - la longueur du syntagme ayant probablement gêné les informateurs dans leur évaluation ici aussi -, ces résultats confirment globalement ceux de la première question en ce sens qu'ils indiquent une très large préférence pour le [a:] allongé et postérieur dans le mot pâte.

\section{Question 3 : prononciation à enseigner dans les cours de français langue étrangère (FLE)}

La dernière question par rapport à ces deux variantes ([a] [a: ]) dans le mot pâte, qui porte sur la prononciation que les informateurs estiment la plus apte à être enseignée dans les cours de FLE donnés en Suisse romande, montre des résultats très similaires à la question 2 (cf. supra).

Premièrement, dans un contexte isolé, l'évaluation du mot pâte aboutit à une large préférence pour la variante [a:], choisie par 93,75\% (90/96) des informateurs, 4,17\% (4/96) seulement préférant la variante [a]. Le modèle de régression indique que cette différence est, ici aussi, hautement significative à $p<0,001$ (cf. figure 117). 
Selon vous, laquelle des deux prononciations du mot suivant serait la plus adaptée à être enseignée à des non-francophones en Suisse ? " pâte "

100
90
80
70
60
50
40
30
20
10
0

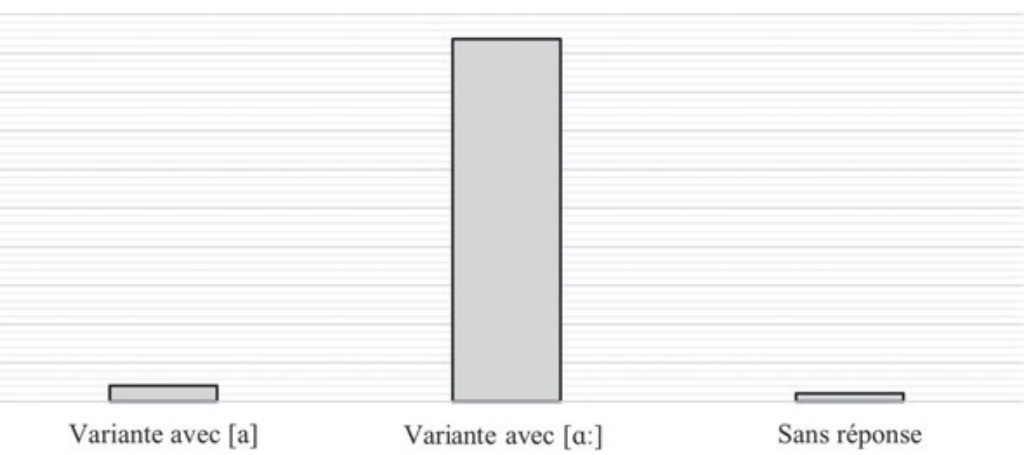

Figure 117 : Voyelle privilégiée (en \%) dans le mot <pâte> pour les cours de français langue étrangère (FLE) $(\mathrm{n}=96)$ - Mots isolés ( $p<0,001$, coef. $=0,63$, e.s. $=0,17, t=3,78)$.

Deuxièmement, dans le contexte du syntagme et plus récemment son usine de pâtes italiennes, les résultats montrent à nouveau des tendances similaires mais quelque peu plus faibles : les informateurs préfèrent le [a:] dans 65,63\% (63/96) et le [a] dans 27,08\% (26/96) des cas, cette différence étant significative à $p<0,001$ (cf. figure 118).

Selon vous, laquelle des deux prononciations du groupe de mots suivant serait la plus adaptée à être enseignée à des non-francophones en Suisse ?

«Et plus récemment son usine de pâtes italiennes »

100
90
80
70
60
50
40
30
20
10
0

$$
90
$$

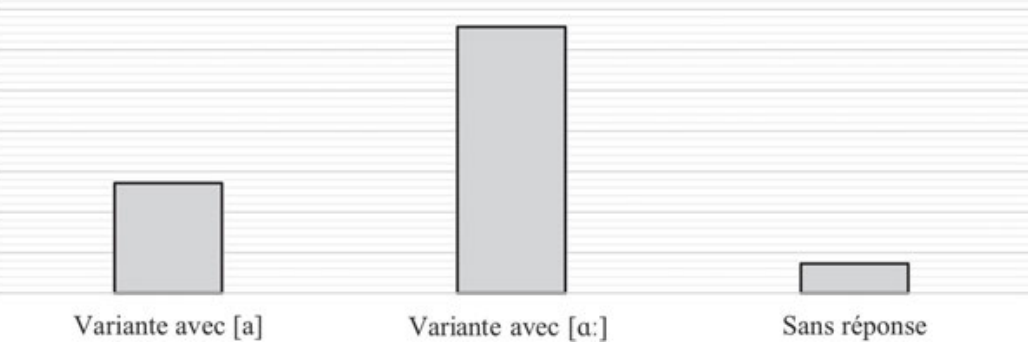

Figure 118 : Voyelle privilégiée (en \%) dans le mot <pâte> pour les cours de français langue étrangère (FLE) $(\mathrm{n}=96)$ - Texte PFC ( $p<0,001$, coef. $=2,07$, e.s. $=0,31, t=6,77)$. 
Le [a:] allongé et postérieur se trouvant en syllabe finale fermée du mot pâte est donc également préféré par une grande majorité des informateurs dans le contexte du texte PFC.

Finalement, les évaluations perceptives effectuées dans le cadre de ces trois questions confirment largement le principal résultat obtenu pour ce trait lors de l'analyse des productions (cf. 5.2.1.1) : le [a:] allongé et postérieur n'est pas seulement utilisé par une majorité des locuteurs-modèles, mais également largement préféré dans les perceptions des Suisses romands. La tendance actuelle à la neutralisation du trait observée dans les productions des présentateurs n'a d'équivalent en perception que dans le cadre de la première question, dans laquelle la tendance plus forte des informateurs âgés à évaluer positivement le [a:] a été mise en évidence. En conclusion, l'opposition /a/ : / $a$ :/ semble donc être bel et bien maintenue dans la norme de prononciation suisse romande en syllabe finale fermée, et ce, indépendamment du type de question posée.

\subsubsection{Opposition /e/: /e:/ en syllabe finale ouverte}

Le deuxième trait soumis aux tests de perception est l'opposition de durée /e/ : /e:/ en syllabe finale ouverte. Rappelons que l'analyse des productions, effectuée à l'exemple des mots pensée (liste de mots PFC) et année (texte PFC), a révélé que l'opposition est encore bien majoritaire parmi les locuteurs-modèles, mais qu'elle se trouve également dans une phase de neutralisation en faveur d'un /e/ bref (cf. 5.2.1.2). Notons par ailleurs que même si dans certains cantons de Suisse romande, l'opposition de durée peut être complétée d'une diphtongaison en /e:j/ (cf. 2.3.3), cette diphtongaison n'a pas été relevée dans l'usage des locuteurs-modèles, raison pour laquelle elle n’a pas été prise en compte dans les présents tests de perception.

\section{Question 1 : évaluation (sans contexte explicite)}

Tout d'abord, les tests effectués sur la base de l'échelle d'évaluation (0\%-100\%) et sans contexte explicite indiquent qu'ici aussi, les informateurs suisses romands préfèrent largement la variante endogène allongée [e:] : les évaluations moyennes affichent en effet un score de 70,23\% (écart-type : 28,46\%) pour la variante [e] et de $92,25 \%$ (écart-type : 13,57\%) pour la variante [e:], la différence entre ces deux moyennes étant hautement significative à $p<0,001$ selon l'analyse de variance (cf. figure 119).

Les résultats liés aux variantes [e] et [e:] du mot année dans le syntagme en fin d'année tiré du texte PFC sont très similaires, avec une préférence pour la variante 
Évaluation de [e] vs [e:] dans <pensée> (liste de mots PFC)

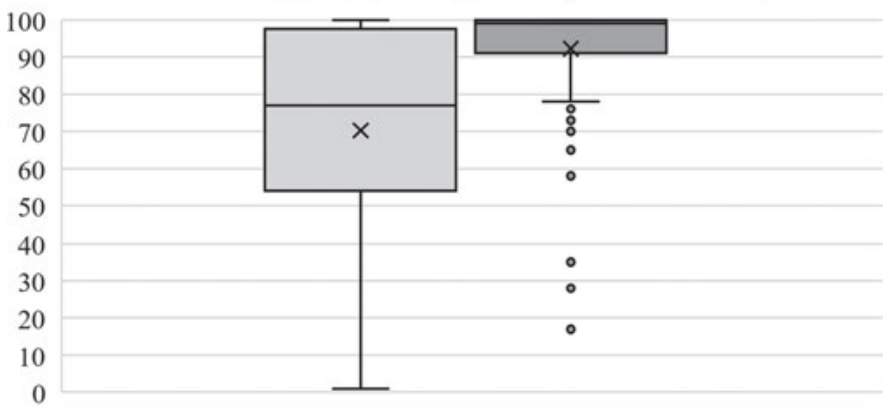

$\square[\mathrm{e}] \square[\mathrm{e}:]$

Figure 119 : Évaluation perceptive (en \%) des voyelles [e] et [e:] par les informateurs suisses romands $(\mathrm{n}=96)$ dans le mot isolé $<$ pensée $>$ (ANOVA : $p<0,001)$.

allongée [e:], qui obtient un score moyen de 87,05\% (écart-type : 17,66\%), contre 75,40\% (écart-type : 25,32\%) pour la variante [e]. Ici aussi, l'analyse de variance montre une différence significative à $p<0,001$ entre ces deux scores moyens (cf. figure 120).
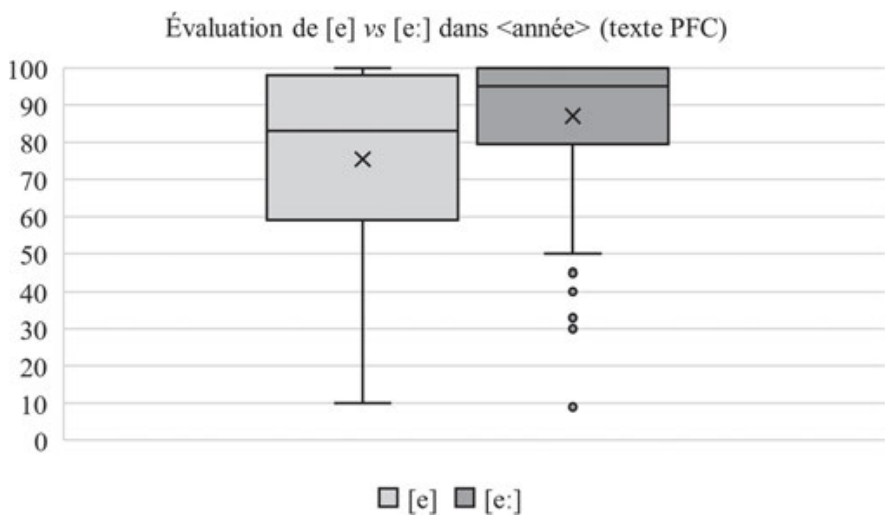

Figure 120 : Évaluation perceptive (en \%) des voyelles [e] et [e:] par les informateurs suisses romands $(\mathrm{n}=96)$ dans le mot $<$ pensée> tiré du texte PFC $($ ANOVA $: p<0,001)$.

Notons qu'aucun effet significatif des facteurs pris en compte (sexe, âge, origine, niveau d'éducation) n’a pu être relevé, ni dans le cas de pensée ni celui d'année $(p>0,05)$. Globalement, les résultats touchant à cette première question, destinée à tester une norme stable et indépendante de la situation, montrent donc une préférence très claire des informateurs suisses romands pour la variante allongée [e:]. 


\section{Question 2 : prononciation idéale des présentateurs de journaux télévisés}

En ce qui concerne la question portant sur la prononciation perçue comme idéale pour un présentateur de télévision, les résultats montrent tout d'abord également une large préférence des informateurs pour la variante allongée [e:] dans le mot isolé pensée : une grande majorité de 79,17\% (76/96) des informateurs choisit en effet cette variante allongée, contre $16,67 \%$ (16/96) pour la variante brève [e], la différence étant significative à $p<0,001$ (cf. figure 121).

Selon vous, comment un présentateur de télévision suisse devrait-il prononcer le mot suivant? «pensée »

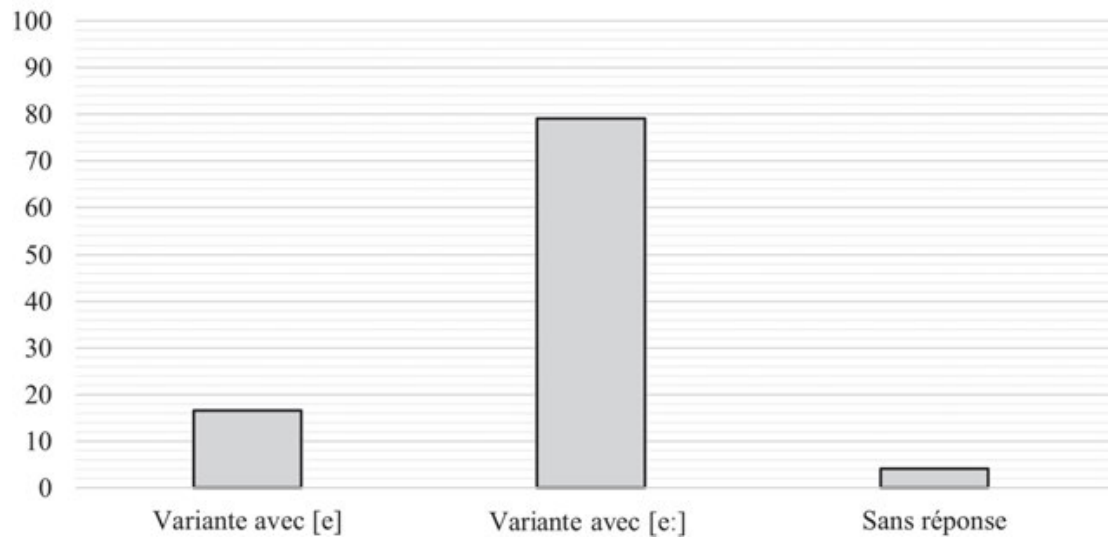

Figure 121 : Voyelle privilégiée (en \%) dans le mot <pensée> pour les présentations de journaux télévisés $(n=96)$ - Mots isolés ( $p<0,001$, coef. $=1,78$, e.s. $=0,21, t=8,68)$.

Cette même question, mais portant cette fois-ci sur le syntagme en fin d'année, montre une tendance à nouveau similaire à privilégier la variante [e:], cette tendance étant cependant moins forte que dans le cas de pensée : en effet, 50,00\% (48/96) des informateurs préfèrent la variante allongée [e:], contre 40,63\% (39/96) pour le [e] bref. Le modèle de régression indique tout de même un niveau de signification de cette différence relativement élevé ( $p<0,01)$ (cf. figure 122).

Notons que, de manière similaire à ce qui a pu être observé dans le cas de l'opposition /a/ : /a:/ (cf. 6.2.1), la légère différence observée entre les évaluations touchant au mot isolé pensée et au syntagme en fin d'année est probablement attribuable à la différence de longueur des deux stimuli soumis aux tests, la longueur du syntagme ayant pu entraîner chez les informateurs certaines difficultés à reconnaître le trait à évaluer. Il est donc vraisemblable que la différence puisse être principalement expliquée par la méthode employée. 
Selon vous, comment un présentateur de télévision suisse devrait-il prononcer le groupe de mots suivant? «En fin d'année »

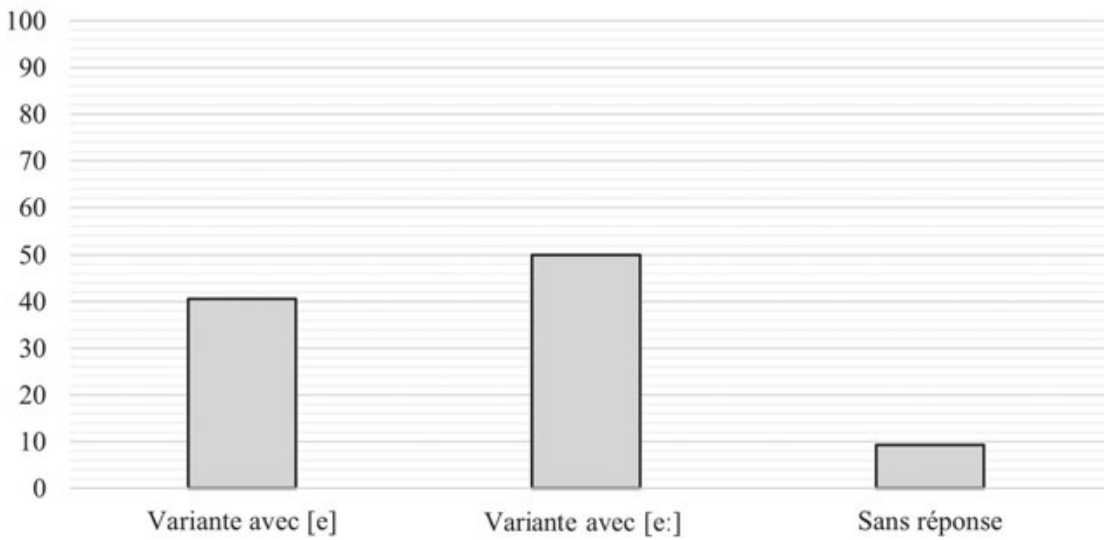

Figure 122 : Voyelle privilégiée (en \%) dans le mot <année> pour les présentations de journaux télévisés $(\mathrm{n}=96)$ - Texte PFC ( $p<0,01$, coef. $=1,04$, e.s. $=0,28, t=3,69)$.

Relevons également que les modèles de régression logistique binomiale appliqués au mot isolé pensée (cf. figure 121) et au syntagme en fin d'année (cf. figure 122) n'indiquent aucun effet significatif des variables sociodémographiques (sexe, âge, origine, niveau d'éducation). Cette deuxième question montre donc globalement des résultats très similaires à la première, avec une forte préférence des informateurs pour la variante allongée [e:].

\section{Question 3 : prononciation à enseigner dans les cours de français langue étrangère (FLE)}

Finalement, la question portant sur la prononciation la plus apte à être enseignée dans les cours de FLE présente des distributions très similaires à celles de la question 2 (cf. supra). Dans le contexte du mot isolé pensée, tout d'abord, les informateurs choisissent à une large majorité $(82,29 \%, 79 / 96)$ la variante allongée [e:], contre seulement $11,46 \%$ (11/96) pour la brève [e]. Sans surprise étant donnée la clarté de ces résultats, la différence entre ces deux taux est hautement significative à $p<0,001$ et aucun effet des variables sociodémographiques n'est observable (cf. figure 123).

Les résultats obtenus par le syntagme en fin d'année indiquent, dans cette question également, une tendance similaire, mais moins forte à privilégier la variante allongée [e:] : 53,15\% (51/96) des informateurs choisissent en effet le [e:] tandis que 41,67\% (40/96) préfèrent le [e], la différence entre ces taux étant significative à $p>0,01$ (cf. figure 124). 
Selon vous, laquelle des deux prononciations du mot suivant serait la plus adaptée à être enseignée à des non-francophones en Suisse ? "pensée »

100

90

80

70

60

50

40

30

20

10

0

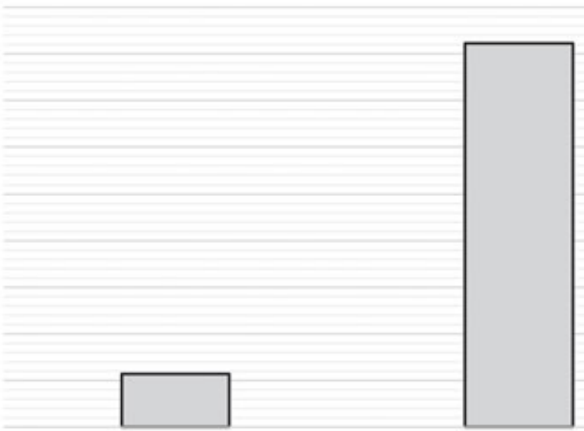

Variante avec $[\mathrm{e}]$

Variante avec [e:]

Sans réponse

Figure 123 : Voyelle privilégiée (en \%) dans le mot <pensée> pour les cours de français langue étrangère (FLE) $(n=96)$ - Mots isolés $(p<0,001$, coef. $=0,88$, e.s. $=0,25, t=3,58)$.

Selon vous, laquelle des deux prononciations du groupe de mots suivant serait la plus adaptée à être enseignée à des non-francophones en Suisse ? « En fin d'année »

100
90
80
70
60
50
40
30
20
10
0

0

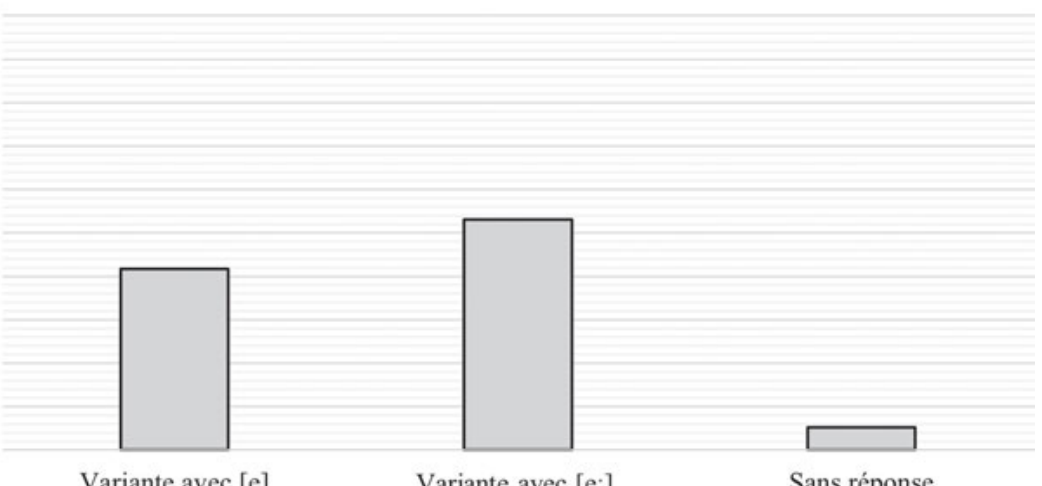

Variante avec [e]

Variante avec [e:]

Sans réponse

Figure 124 : Voyelle privilégiée (en \%) dans le mot <année> pour les cours de français langue étrangère (FLE) $(n=96)$ - Texte PFC ( $p<0,01$, coef. $=3,10$, e.s. $=1,00, t=3,09)$.

Mentionnons, ici aussi, que la tendance légèrement moins forte en faveur de la variante allongée [e:] est probablement due au problème méthodologique de la longueur du stimulus en fin d'année par rapport à celle du mot isolé pensée. La différence entre les résultats présentés dans les figures 123 et 124 semble donc relativement négligeable. 
En conclusion, les présents résultats ont clairement confirmé la préférence des Suisses romands pour la variante allongée [e:], déjà observée dans les productions d'une majorité des locuteurs-modèles (cf. 5.2.1.2). L'opposition de durée /e/ : /e:/ en syllabe finale ouverte semble donc faire partie de la norme de prononciation actuelle en Suisse romande et ce, à nouveau, indépendamment du type de question posée. Cependant, au contraire des résultats touchant aux productions, ces perceptions n'ont pas révélé d'indices d'un changement en cours en direction d'une neutralisation de l'opposition aboutissant à un [e] bref. Les perceptions observées ici ont donc tendance à renforcer l'état actuel de l'opposition et, au contraire des productions, elles ne suggèrent pas de neutralisation.

\subsubsection{Opposition /e/ : / $\varepsilon /$ en syllabe finale ouverte}

L'opposition /e/ : / $\varepsilon /$ en syllabe finale ouverte est le troisième trait soumis aux tests de perception. Les analyses de production effectuées à l'exemple du mot isolé (je) pourrai (cf. 5.2.1.3) ont montré que cette opposition continue à être réalisée par une majorité des locuteurs-modèles, mais qu'elle montre parallèlement une tendance à la neutralisation en faveur $d u[\varepsilon]$, en particulier parmi les locuteurs-modèles les plus jeunes. Il a été noté que les règles de décodage graphie-phonie pourraient expliquer en partie cette neutralisation, étant donné que le digramme <ai> correspond en général à un $[\varepsilon]$ en français de référence (cf. Lyche/Østby 2009, 212). Ces résultats seront soumis à l'épreuve d'évaluations perceptives, à nouveau à l'exemple du mot (je) pourrai. Rappelons, ce faisant, qu'étant donné qu'aucune paire minimale appropriée n’a pu être trouvée dans le texte PFC pour les analyses de production, les tests de perception ont également été restreints aux mots isolés.

\section{Question 1 : évaluation (sans contexte explicite)}

Les résultats de l'évaluation perceptive sans contexte explicite montrent, tout d'abord, une large préférence pour la variante endogène du [e] fermé dans la forme testée je pourrai : cette variante obtient en effet un score de 83,02\% (écarttype : 25,58\%), contre 44,85\% (écart-type : 40,24\%) pour la variante [c], la différence étant significative à $p<0,001$ selon l'analyse de variance. Soulignons ici l'écart-type considérable engendré par les évaluations de la variante $[\varepsilon]$, qui indique une forte insécurité chez les informateurs : dans leur majorité, ces derniers ne semblent manifestement pas associer cette variante à la terminaison $<$-ai $>$ du futur simple < pourrai $>$ (cf. figure 125). 
Évaluation de $/ \mathrm{e} / v s / \varepsilon /$ dans $<\mathrm{je}$ pourrai $>$ (liste de mots $\mathrm{PFC}$ )

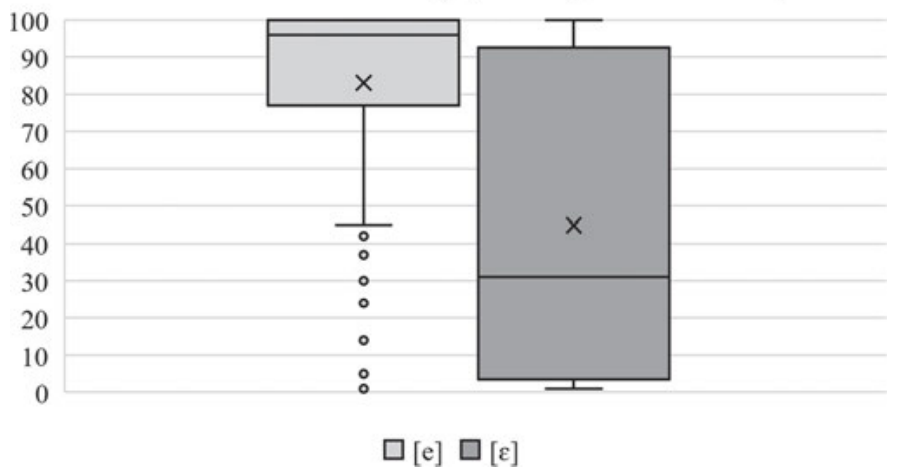

Figure 125 : Évaluation perceptive (en \%) des voyelles [e] et $[\varepsilon]$ par les informateurs suisses romands $(\mathrm{n}=96)$ dans le mot isolé <(je) pourrai $>$ (ANOVA : $p<0,001)$.

L'analyse de variance montre, par ailleurs, un effet positif des jeunes informateurs (facteur de l'âge) sur les évaluations de la variante ouverte $[\varepsilon](F=5,73$, $p<0,01)$, mais pas sur les évaluations de la variante fermée [e] $(F=2,37, p>0,05)$. Contrairement à la majorité des informateurs, les plus jeunes semblent ainsi présenter une tendance à mieux accepter la variante ouverte $[\varepsilon]$ pour exprimer le futur, ce qui correspond aux résultats obtenus dans les productions (cf. 5.2.1.3), qui ont montré que les jeunes ont plus souvent tendance à réaliser le <-ai> de $<$ pourrai $>$ comme un $[\varepsilon]$ ouvert.

\section{Question 2 : prononciation idéale de présentateurs de journaux télévisés}

Les résultats de la question portant sur la prononciation perçue comme idéale pour un présentateur de télévision sont très similaires, avec une large préférence chez 78,13\% (75/96) des informateurs pour la variante fermée [e] (78,13\%, 75/96), contre $21,88 \%$ (21/96) pour la variante ouverte [ $\varepsilon]$. La différence entre ces deux taux est significative à $p>0,001$. Notons par ailleurs que le modèle de régression montre que l'âge a, ici aussi, un effet significatif sur les résultats : si les informateurs de 20 à 39 ans ne présentent aucune différence notable par rapport à ceux âgés de 40 à 59 ans $(p>0,05)$, ils montrent une tendance significativement plus forte que les plus de 60 ans à choisir la variante ouverte $[\varepsilon](p<0,05)$. Ces résultats sont résumés dans la figure 126.

Ainsi, les résultats des évaluations de la première question se confirment également dans cette deuxième question, avec une large préférence des informateurs pour la variante fermée [e], accompagnée d'une tendance des plus jeunes à mieux accepter la réalisation ouverte $[\varepsilon]$. Ces résultats correspondent ainsi, à nouveau, aux deux mêmes tendances observées dans les productions (cf. 5.2.1.3). 
Selon vous, comment un présentateur de télévision suisse devrait-il prononcer le mot suivant ? « (je) pourrai »

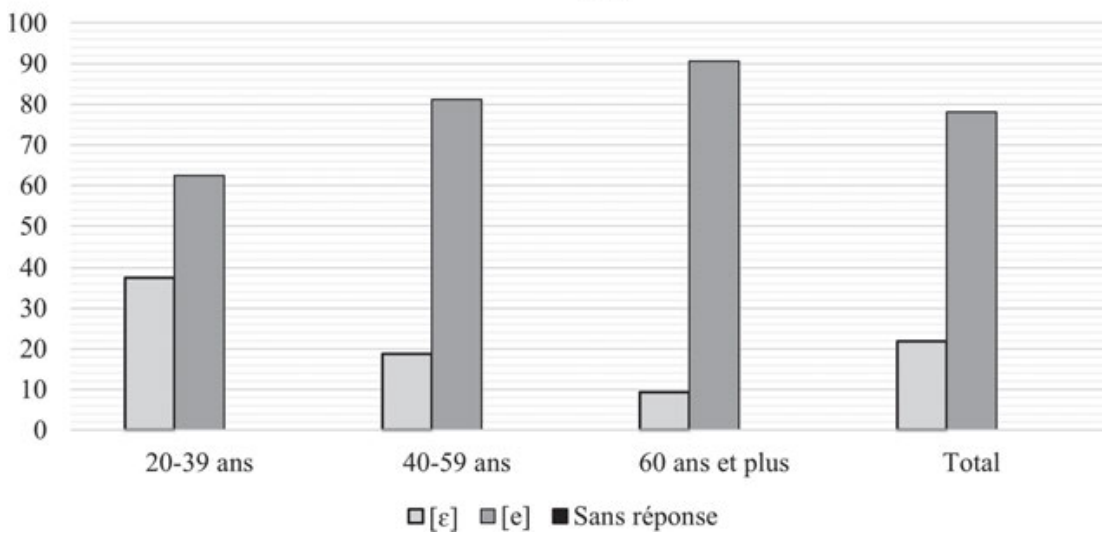

Figure 126 : Voyelle privilégiée (en \%) dans le mot <(je) pourrai> pour les présentations de journaux télévisés $(\mathrm{n}=96)$ - Mots isolés ; réponses présentées dans leur globalité $(p<0,001$, coef. $=1,98$, e.s. $=0,18, t=10,85$ ) ainsi que selon l'âge des informateurs (âge : $20-39$ ans vs. $40-59$ ans : $p>0,05$, coef. $=0,06$, e.s. $=0,12, t=0,48 ; 20-39$ ans vs. 60 ans et plus : $p<0,05$, coef. $=0,26$, e.s. $=0,12, t=2,14)$.

\section{Question 3 : prononciation à enseigner dans les cours de français langue étrangère (FLE)}

Finalement, la tendance à privilégier la variante [e] se confirme également dans la question sur la prononciation perçue par les informateurs comme étant la plus apte à être enseignée dans les cours de FLE : en effet, 79,17\% (76/96) d'entre eux préfèrent cette variante [e], tandis que $18,75 \%$ (18/96) choisissent la variante $[\varepsilon]$, cette différence de taux étant hautement significative à $p<0,001$ (cf. figure 127).

Dans le cas présent, les variables sociodémographiques prises en compte dans le modèle de régression ne montrent aucun effet significatif $(p>0,05)$. Relevons tout de même une légère tendance détectée par le modèle : le comportement de la catégorie d'âge des 20 à 39 ans diffère de celui des classes d'âge 40-59 ans ( $p<0,1$, coef. $=0,33$, e.s. $=0,17, t=1,95)$ et plus de 60 ans $(p<0,1$, coef. $=0,30$, e.s. $=0,16, t=1,83)$, cette tendance $(p<0,1)$ n'étant cependant pas significative selon le seuil de 0,05 .

Finalement, les résultats des tests de perception ont confirmé les deux tendances décelées dans les productions des locuteurs-modèles (cf. 5.2.1.3) : d'une part, la variante fermée [e] est largement préférée dans la terminaison du futur simple (pourrai), confirmant ainsi l'appartenance bien établie de l'opposition $/ \mathrm{e} /: / \varepsilon /$ (utilisée pour différencier certains temps et modes) à la norme de prononciation actuelle en Suisse romande. D'autre part, la tendance des jeunes locuteurs-modèles à l'utilisation accrue de la variante ouverte $[\varepsilon]$ pour exprimer 
Selon vous, laquelle des deux prononciations du mot suivant serait la plus adaptée à être enseignée à des non-francophones en Suisse ?

«(je) pourrai »

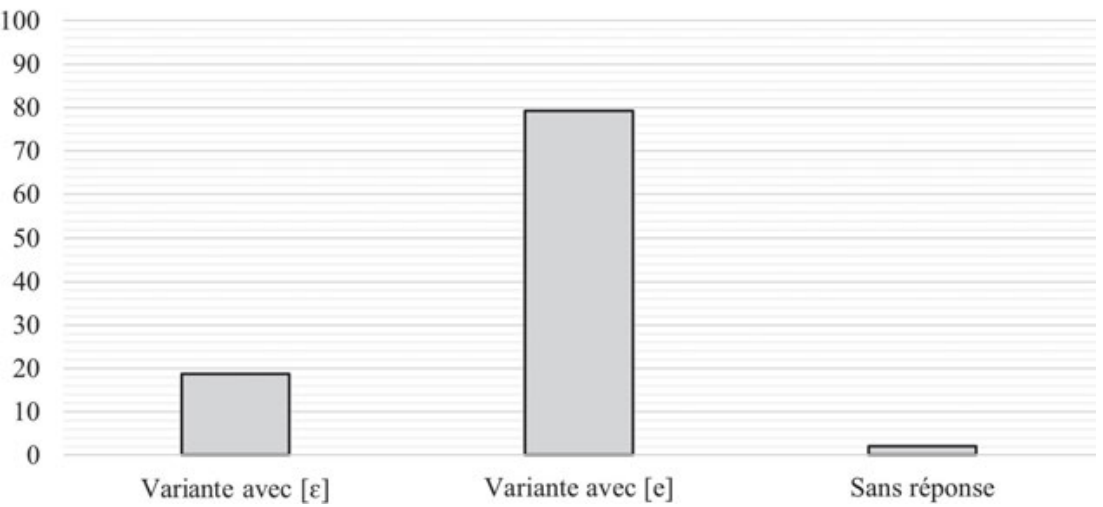

Figure 127 : Voyelle privilégiee (en \%) dans le mot <(je) pourrai> pour les cours de français langue étrangère (FLE) $(\mathrm{n}=96)$ - Mots isolés $(p<0,001$, coef. $=0,88$, e.s. $=0,25, t=3,58)$.

un futur simple se traduit par les taux supérieurs d'acceptation de cette même variante au sein de la catégorie des 20 à 39 ans dans les tests de perception.

\subsubsection{Préférence de la diérèse à la synérèse}

Le quatrième trait soumis aux tests de perception concerne la réalisation des hiatus sous forme de diérèse ou de synérèse. À ce propos, l'analyse des productions, effectuée à l'exemple des mots muette (liste de mots PFC) et habituels (texte PFC), a montré que les présentateurs suisses romands - contrairement aux locuteurs romands « ordinaires » (cf. Andreassen/Maître/Racine 2010, 224) - ne font pas un usage majoritaire de la diérèse (cf. 5.2.1.4). Ce résultat pourrait a priori inciter à ne pas prendre en compte la préférence de la diérèse dans la définition de la norme de prononciation suisse romande, mais il devra encore être mis à l'épreuve des données relatives aux perceptions.

\section{Question 1 : évaluation (sans contexte explicite)}

Les résultats de la première question (évaluation perceptive des stimuli sans mention explicite de contexte) présentent une tendance très claire en faveur de la diérèse, c'est-à-dire la variante considérée comme endogène (cf. Andreassen/ Maître/Racine 2010, 224) : dans le mot isolé muette, la réalisation du hiatus avec 
diérèse ([my.ct]) obtient en effet un score de 88,45\% (écart-type : 18,69\%), contre 45,77\% (écart-type : 31,56\%) pour la réalisation avec synérèse ([muદt]), la différence entre ces deux scores moyens étant significative à $p<0,001$ (cf. figure 128).

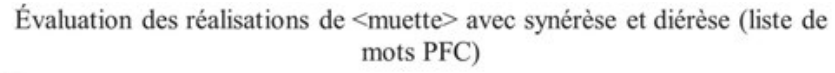
mots PFC)

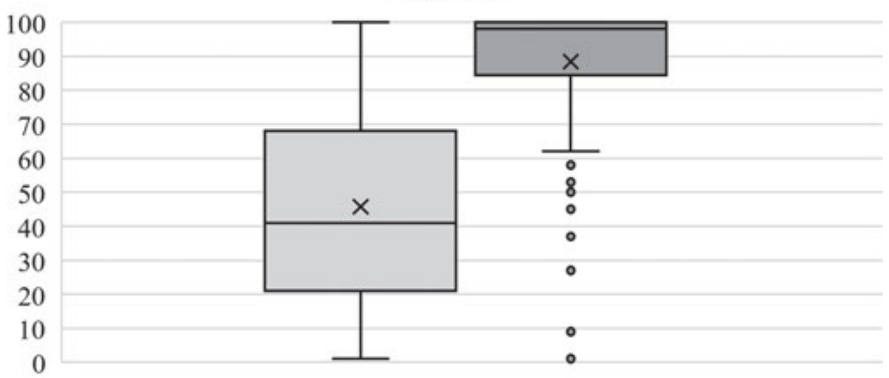

\section{Synérèse $\square$ Diérèse}

Figure 128 : Évaluation perceptive effectuée par les informateurs suisses romands (en \%) de la diérèse et de la synérèse dans le mot isolé <muette> $(\mathrm{n}=96)($ ANOVA $: p<0,001)$.

La tendance est similaire pour l'évaluation du hiatus dans le mot habituels, tiré du texte PFC et soumis à l'évaluation dans le cadre du syntagme car le Premier Ministre, lassé des circuits habituels, mais elle est moins évidente : la variante avec diérèse ([abity.cl]) obtient en effet un score de 83,35\% (écart-type : 19,90), contre 77,41\% (écart-type : $23,45 \%$ ) pour la variante avec synérèse ([abitucl]), cette différence de taux étant significative, mais à un niveau moins élevé ( $p<0,05)$ (cf. figure 129).

\section{Évaluation des réalisations de $<$ habituels $>$ avec synérèse et diérèse (texte}

PFC)

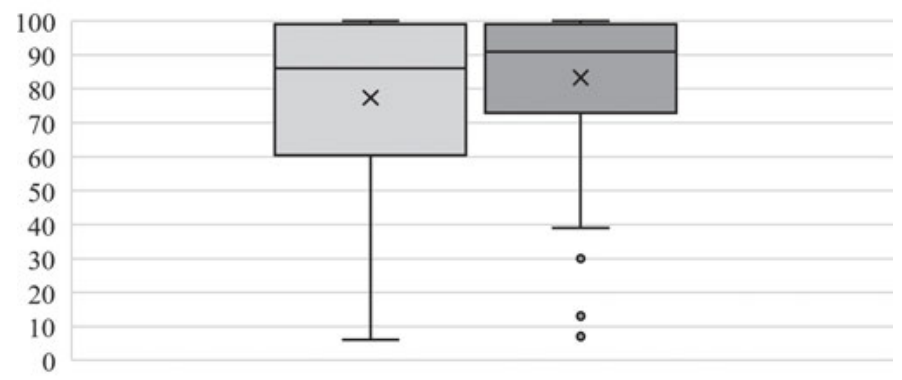

\section{Synérèse $\square$ Diérèse}

Figure 129 : Évaluation perceptive (en \%) effectuée par les informateurs suisses romands de la diérèse et de la synérèse dans le mot <habituels> tiré du texte $\operatorname{PFC~}(\mathrm{n}=96)($ ANOVA : $p<0,05)$. 
Notons que la différence entre les évaluations du mot isolé (muette) ou présenté dans un contexte syntagmatique (habituels) peut probablement être à nouveau attribuée à des causes méthodologiques, la longueur du syntagme ayant sans doute eu une influence chez les informateurs sur la reconnaissance du trait à évaluer. Ajoutons par ailleurs qu'aucun effet significatif des quatre facteurs sociodémographiques pris en compte (sexe, âge, origine, niveau d'éducation) n'a pu être relevé.

\section{Question 2 : prononciation idéale de présentateurs de journaux télévisés}

Les résultats de la deuxième question, qui porte sur la prononciation idéale des présentateurs de journaux télévisés, montrent des tendances similaires : dans le cas du mot isolé muette, 95,83\% (92/96) des informateurs préfèrent la variante avec diérèse, contre seulement 3,13\% (3/96) pour la synérèse. Sans surprise, cette différence de taux est hautement significative $(p<0,001)$, alors que le modèle de régression n'indique aucun effet significatif des données sociodémographiques (cf. figure 130).

Selon vous, comment un présentateur de télévision suisse devrait-il prononcer le mot suivant ? «muette »

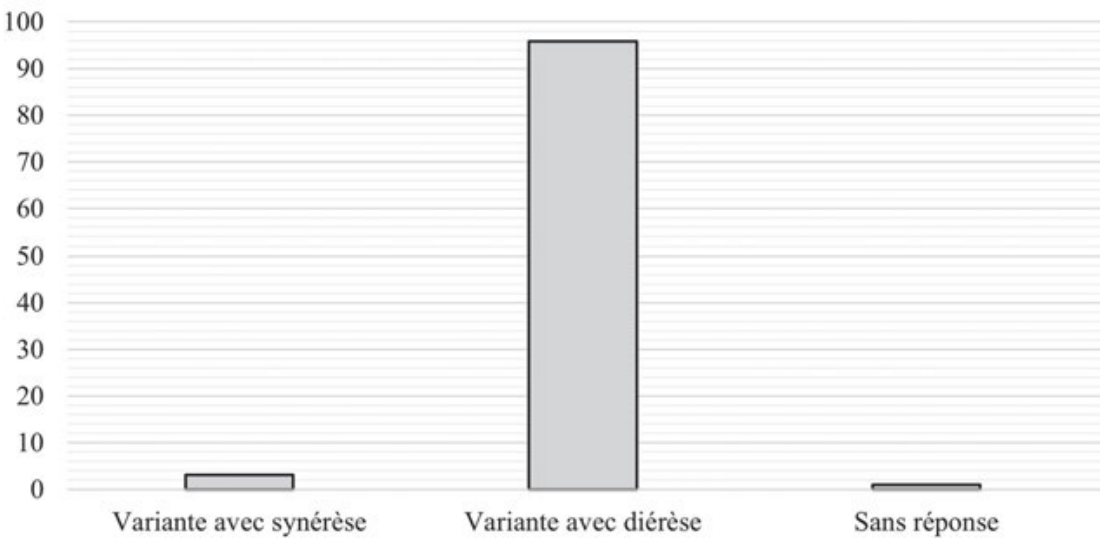

Figure 130 : Variante privilégiée (en \%) dans le mot <muette> pour les présentations de journaux télévisés $(\mathrm{n}=96)$ - Mots isolés ( $p<0,001$, coef. $=1,98$, e.s. $=0,10, t=20,42)$.

Le cas du mot habituels dans le contexte du syntagme car le Premier Ministre, lassé des circuits habituels ne confirme cependant pas cette tendance, les résultats ne montrant aucune véritable tendance : les variantes avec diérèse $(47,92 \%, 46 / 96)$ et synérèse $(45,83 \%, 44 / 96)$ sont choisies à des taux quasiment identiques, la différence minime n'étant, sans surprise, pas significative ( $p>0,05)$ (cf. figure 131). 
Selon vous, comment un présentateur de télévision suisse devrait-il prononcer le groupe de mots suivant?

«Car le Premier Ministre, lassé des circuits habituels »

100

90

80

70

60

50

40

30

20

10

0

Figure 131 : Variante privilégiée (en \%) dans le mot <habituels> pour les présentations de journaux télévisés $(\mathrm{n}=96)$ - Texte PFC $(p>0,05$, coef. $=0,69$, e.s. $=0,41, t=1,70)$.

Étant donné que les oppositions /a/ : /a:/ (cf. 5.2.1) et /e/ : /e:/ (cf. 5.2.2) ont montré une tendance similaire à entrainer des évaluations plus confuses pour les syntagmes, il semble à nouveau pertinent d'interpréter la différence entre les taux du mot isolé muette et du mot habituels dans le syntagme car le Premier Ministre, lassé des circuits habituels comme résultante du faible degré de saillance de ce trait : visiblement, la longueur du syntagme a, ici aussi, suscité chez les informateurs des problèmes pour distinguer le trait à évaluer. Les résultats relatifs aux syntagmes révèlent ainsi que la différence entre synérèse et diérèse s'estompe perceptivement dans des stimuli plus longs. En d'autres termes, il est possible que cette différence ne présente qu'un faible degré de saillance dans les perceptions des informateurs, de sorte que la présence de l'une ou de l'autre variante n'influence pas significativement les évaluations du stimulus. Cette observation secondaire devrait cependant encore être vérifiée dans de futures études similaires.

\section{Question 3 : prononciation à enseigner dans les cours de français langue étrangère (FLE)}

Pour conclure, les résultats de la troisième question, qui porte sur la prononciation à enseigner dans les cours de FLE, présentent des distributions très similaires à celles de la deuxième question, et ce, en ce qui concerne aussi bien le mot muette (liste de mots PFC) que le mot habituels (texte PFC). Dans le cas du mot isolé, tout d'abord, 94,79\% (91/96) des informateurs préfèrent la variante avec diérèse ([abity.cl]), contre seulement 4,17\% (4/96) pour celle avec synérèse 
([abitucl]). La différence entre ces deux taux s'avère hautement significative à $p<0,001$ (cf. figure 132).

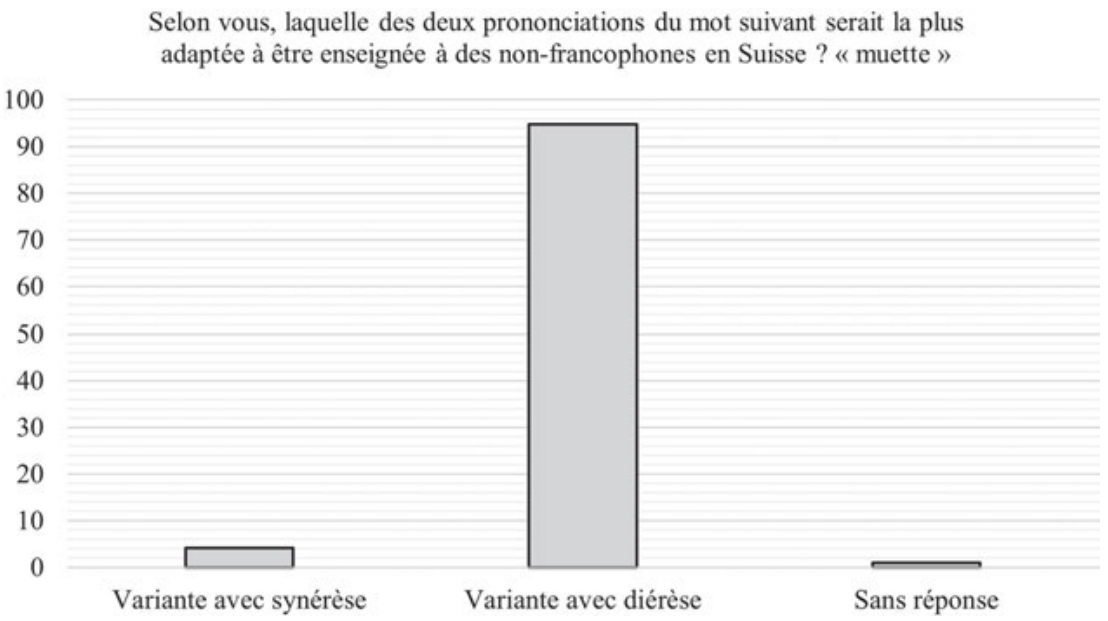

Figure 132 : Variante privilégiée (en \%) dans le mot <muette> pour les cours de français langue étrangère (FLE) $(\mathrm{n}=96)$ - Mots isolés ( $p<0,001$, coef. $=1,08$, e.s. $=0,14, t=7,63)$.

Les résultats concernant le syntagme car le Premier Ministre, lassé des circuits habituels présentent, pour leur part, la même hésitation que pour la question 2. En effet, seuls 44,79\% (43/96) des informateurs choisissent la variante avec diérèse, contre un taux très similaire de 40,63\% (39/96) préfèrant la variante avec synérèse. Sans surprise, la différence entre ces taux n'est pas significative $(p>0,05)$ (cf. figure 133).

Étant donnée la différence observable ici aussi entre les résultats touchant au mot isolé muette et au syntagme car le Premier Ministre, lassé des circuits habituels, l'interprétation de la longueur de ce dernier comme élément perturbateur posant des problèmes aux informateurs dans leurs évaluations semble se confirmer, d'autant plus que dans le cas présent, les informateurs ayant sauté la question sont particulièrement nombreux $(14,58 \%, 14 / 96)$. De même que pour la question 2 , ces résultats paraissent par ailleurs confirmer le manque de saillance de la différence entre diérèse et synérèse, de sorte que même dans une comparaison directe de stimuli, la différence passe apparemment inaperçue pour les informateurs.

En conclusion, au vu des résultats du mot isolé muette, dans un contexte d'opposition directe entre synérèse et diérèse, la seconde semble largement préférée à la synérèse. Ce résultat va à l'encontre des résultats sur les productions, qui ont montré que la majorité des présentateurs ne recourt pas à la diérèse. Deux 
Selon vous, laquelle des deux prononciations du groupe de mots suivant serait la plus adaptée à être enseignée à des non-francophones en Suisse ? "Car le Premier Ministre, lassé des circuits habituels"

100

90

80

70

60

50

40

30

20

10

0

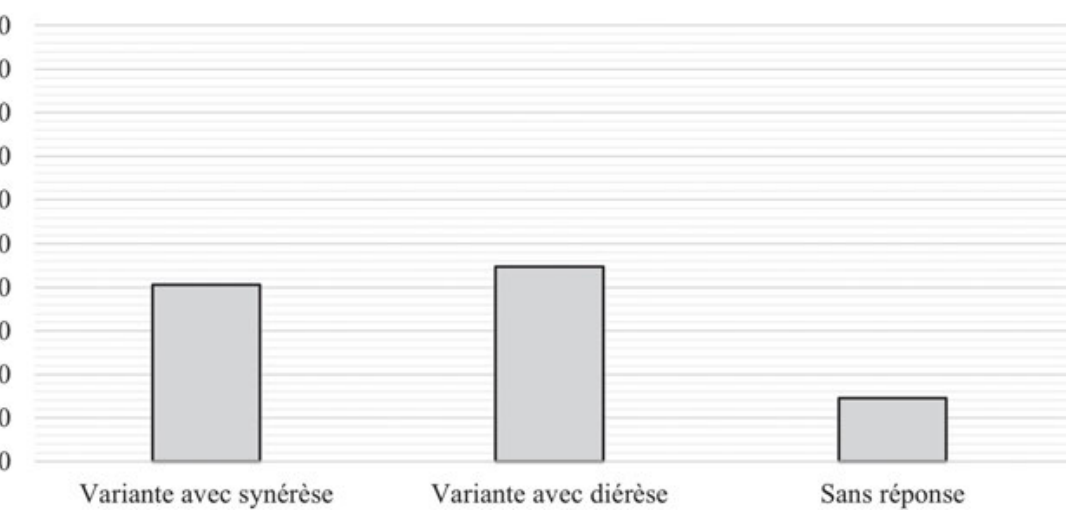

Figure 133 : Variante privilégiée (en \%) dans le mot <habituels> pour les cours de français langue étrangère $(\mathrm{FLE})(\mathrm{n}=96)$ - Texte $\mathrm{PFC}(p>0,05$, coef. $=1,16$, e.s. $=0,87, t=1,34)$.

réserves doivent cependant être émises par rapport à ce résultat : premièrement, selon l'interprétation des résultats du syntagme dans les questions 2 et 3, la différence entre synérèse et diérèse ne semble pas particulièrement saillante, étant donné qu'elle n'est pas perçue par les informateurs lorsque les stimuli testés sont plus longs. Cette observation pourrait remettre en cause la pertinence de ce trait dans une définition de la norme de prononciation suisse romande. Deuxièmement, l'on pourrait également se demander dans quelle mesure la différence d'évaluation entre diérèse et synérèse pourrait être le fruit non de perceptions, mais plutôt de représentations (cf. 3.1), les informateurs étant éventuellement tentés de séparer les syllabes dans un hiatus visible au niveau graphique (p. ex.

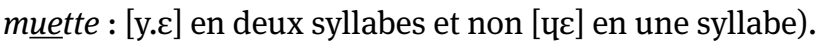

Finalement, du fait des résultats contradictoires des productions et des perceptions, ainsi que des réserves énoncées par rapport à la saillance de ce trait et à la nature (perceptive ou représentative) des évaluations, ce trait ne peut - à ce jour être considéré comme faisant partie de la norme de prononciation suisse romande. Des études ultérieures prenant en compte ces divers problèmes (principalement méthodologiques) sont nécessaires pour apporter davantage de clarté sur ce point.

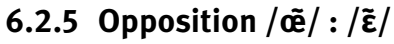

Le dernier trait soumis aux tests de perception effectués en Suisse romande concerne l'opposition /õe/ : / $\tilde{\varepsilon} /$, testée à l'exemple des mots brun et un. Rappelons 
que l'analyse des productions a montré deux résultats parallèles (cf. 5.2.2) : d'une part, l'opposition semble bien se maintenir dans la paire minimale brin $\sim$ brun. D’autre part, dans le mot un, contexte de potentielle apparition le plus fréquent

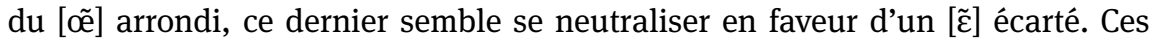
deux résultats seront vérifiés ci-dessous sur la base de perceptions des informateurs suisses romands.

\section{Question 1 : évaluation (sans contexte explicite)}

Dans le cadre de la première question, les informateurs étaient invités à évaluer à nouveau sans mention d'un contexte d'évaluation - les prononciations de brun (mot isolé) et un (dans le syntagme d'un autre côté) avec [õ̃] arrondi et [c̃] écarté. Dans les deux cas, les évaluations des deux variantes ne présentent aucune différence significative selon l'analyse de variance $(p>0,05)$ : dans le cas du mot isolé

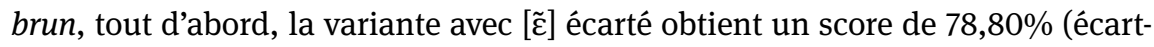
type : $24,46 \%$ ), alors que la variante avec [œẽ] arrondi totalise un score de $83,08 \%$ (écart-type : 22,25\%) (cf. figure 134).

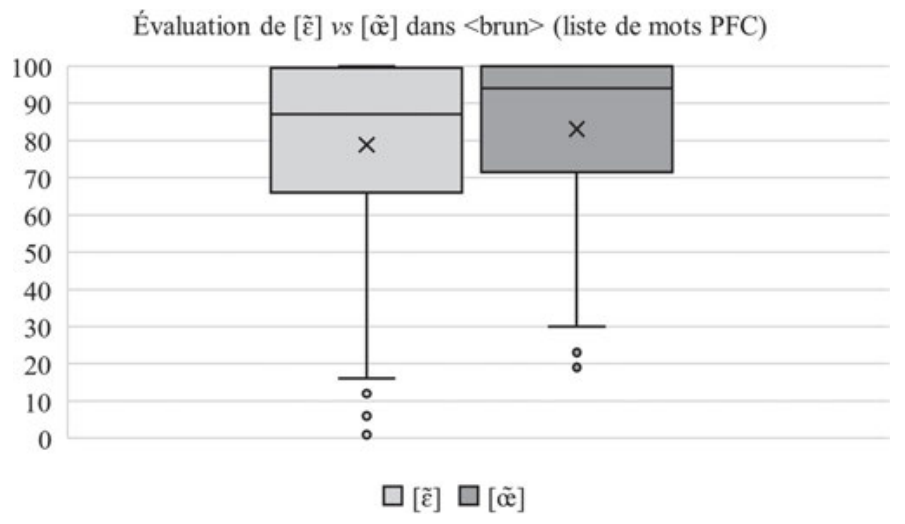

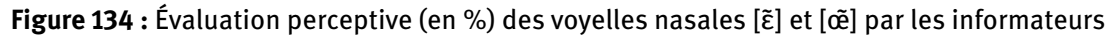
suisses romands $(n=96)$ dans le mot isolé <brun> (ANOVA : $p>0,05)$.

Dans le cas du déterminant un dans le syntagme d'un autre côté, la variante avec

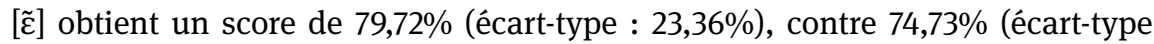
25,43\%) pour celle avec [ø̃] arrondi (cf. figure 135).

Rappelons ici que les évaluations effectuées dans le cadre de cette première question ont été faites séparément pour chacune des variantes, au contraire des questions 2 et 3, dans lesquelles il s'agissait de choisir la variante préférée parmi deux possibilités de réponse, c'est-à-dire dans une comparaison directe des deux 


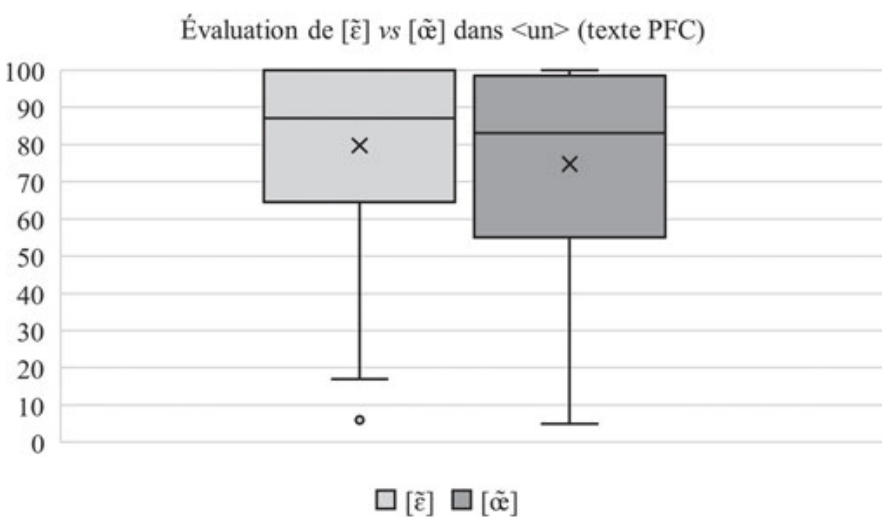

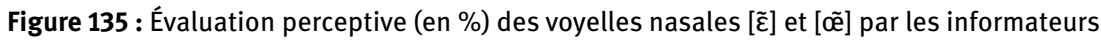
suisses romands $(\mathrm{n}=96)$ dans le mot $<$ un> tiré du texte PFC (ANOVA $: p>0,05)$.

stimuli. Cette particularité méthodologique conjuguée à la non-signification statistique des résultats pourrait indiquer, de manière parallèle à ce qui a pu être observé dans le cas de la différence entre diérèse et synérèse (cf. 6.2.4), que les informateurs ne perçoivent pas de différence entre les variantes lorsque les stimuli ne sont pas comparés directement. En d'autres termes, il est probable que dans les perceptions des informateurs, l'opposition soit, comme dans les productions, en voie de neutralisation et perde simultanément en saillance.

\section{Question 2 : prononciation idéale des présentateurs de journaux télévisés}

La question touchant à la prononciation perçue comme idéale pour un présentateur de télévision obtient des résultats plus complexes. Tout d'abord, contrairement à ce qui est apparu dans les productions des locuteurs-modèles (cf. 5.2.2), les résultats montrent d'une manière générale que la variante écartée [च̃] est également privilégiée dans le mot brun : une majorité de 53,13\% (51/96) des informateurs l'ont en effet choisie, tandis que 35,42\% (34/96) d'entre eux préféraient la variante arrondie [õ̃], la différence entre ces deux taux étant significative à $p<0,01$ (cf. figure 136).

Le modèle de régression montre cependant que ce résultat global doit être nuancé en fonction de l'âge : il indique une différence significative entre la catégorie des informateurs les plus âgés (60 ans et plus) et les plus jeunes (20-39 ans) $(p<0,05)$. Les plus de 60 ans préfèrent en effet en majorité le [õ̃] arrondi $(56,25 \%$, 18/32), alors que les 20 à 39 ans et les 40 à 59 ans privilégient majoritairement le

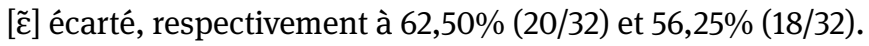

Ainsi, la combinaison des résultats globaux et de cet effet de l'âge montre que l'opposition est engagée dans un processus de changement - un résultat 
Selon vous, comment un présentateur de télévision suisse devrait-il prononcer le mot suivant? «brun »

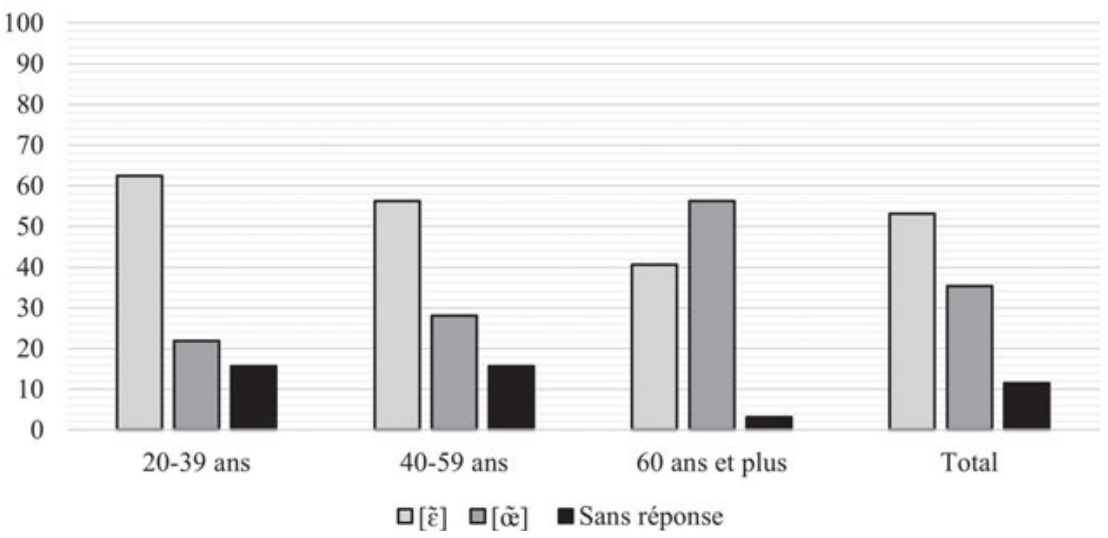

Figure 136 : Voyelle privilégiée (en \%) dans le mot <brun> pour les présentations de journaux télévisés $(\mathrm{n}=96)$ - Mots isolés ; réponses présentées dans leur globalité $(p<0,01$, coef. $=0,94$, e.s. $=0,29, t=3,31$ ) ainsi que selon l'âge des informateurs (âge : $20-39$ ans vs. $40-59$ ans : $p>0,05$, coef. $=0,03$, e.s. $=0,19, t=0,14 ; 20-39$ ans vs. 60 ans et plus : $p<0,05$, coef. $=0,37$, e.s. $=0,18, t=1,98$ ).

confirmant ceux des productions - et que chez les locuteurs, majoritaires en

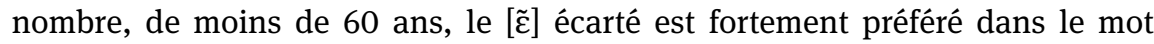
brun - un résultat allant à l'encontre des productions, qui ont montré la résistance de brun au processus de neutralisation. Ce résultat peut être interprété de deux façons : il se pourrait qu'au cours du processus de changement, les perceptions soient " en avance » sur les productions, l'opposition existant encore dans les productions de la paire minimale brun $\sim$ brin alors que dans les perceptions, elle est neutralisée également dans cette paire. Notons ici que pour l'opposition $/ \mathrm{e} /: / \varepsilon /$ à Paris, on observe un changement en sens inverse (les productions y sont à un stade plus avancé que les perceptions dans le processus aboutissant à /e/). Cette différence peut s'expliquer par le fait qu'il s'agit vraisemblablement de deux types de changement différents : alors que dans le cas de /e/ : / $\varepsilon /$ à Paris, le changement vers un /e/ unique semble être interne (les productions devançant les perceptions), dans le cas de /õ̃/: / $\tilde{\varepsilon} /$ en Suisse romande, l'évolution vers un $/ \tilde{\varepsilon} /$ unique parait être due au contact avec le français parisien (les perceptions devançant les productions). D'autre part, il pourrait également s'agir d'un certain « conservatisme » linguistique des locuteurs-modèles, qui maintiennent l'opposition alors que la majorité des locuteurs ordinaires ne la perçoivent plus,

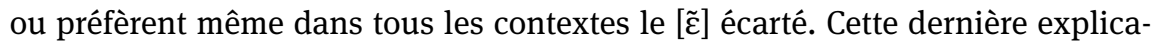
tion semble plus probable. 
Les résultats du déterminant un dans le syntagme d'un autre côté, pour leur part, montrent tout d'abord que les évaluations globales des deux variantes ne se différencient pas significativement l'une de l'autre. En effet, alors que 41,67\%

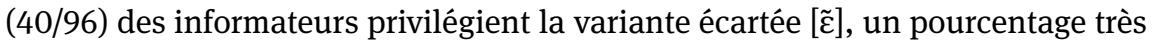
similaire de 40,63\% (39/96) d'entre eux préfère la variante arrondie [õ]], cette différence n'étant pas significative $(p>0,05)$. Les résultats montrent pourtant ici aussi une différence significative, entre la catégorie des informateurs les plus âgés (60 ans et plus) et celle des plus jeunes (20-39 ans) $(p<0,05)$ : les plus âgés

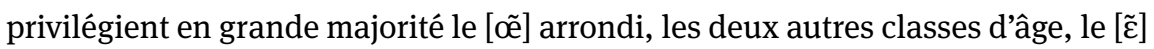
écarté (cf. figure 137).

Selon vous, comment un présentateur de télévision suisse devrait-il prononcer le groupe de mots suivant ? « D'un autre côté »

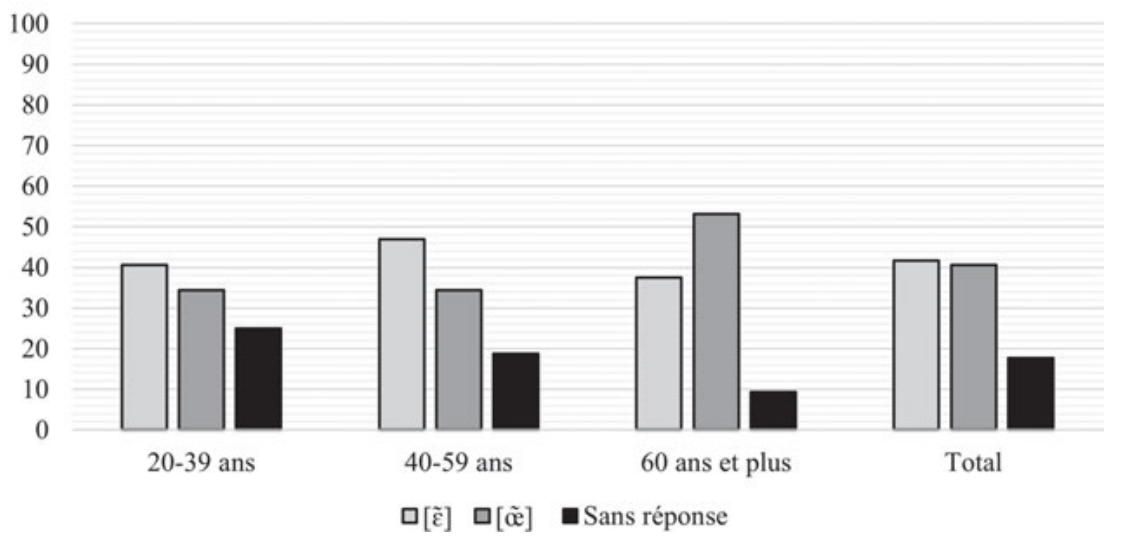

Figure 137 : Voyelle privilégiée (en \%) dans le mot <un> pour les présentations de journaux télévisés $(n=96)$ - Texte PFC ; réponses présentées dans leur globalité $(p>0,05$, coef. $=0,09$, e.s. $=0,14, t=0,65$ ) ainsi que selon l'âge des informateurs (âge : 20-39 ans vs. 40-59 ans : $p>0,05$, coef. $=0,25$, e.s. $=0,19, t=1,34 ; 20-39$ ans vs. 60 ans et plus : $p<0,05$, coef. $=0,43$, e.s. $=0,19, t=2,19)$.

Même si ce résultat global n'est pas significatif, les tendances pour chacune des tranches d'âge sont donc similaires à celles observées dans le cas du mot isolé brun : la préférence pour les réalisations arrondies [õ] est plutôt attribuable aux informateurs les plus âgés, alors que les informateurs de moins de 60 ans préfèrent en moyenne la variante écartée [ $\tilde{\varepsilon}]$. De même que pour les oppositions /a/ : /a:/ (cf. 6.2.1) et /e/ : /e:/ (cf. 6.2.2) ou les différences entre diérèse et synérèse (cf. 6.2.3), cette tendance moins marquée dans l'évaluation des deux variantes dans le syntagme (d'un autre côté) semble à nouveau résulter de la longueur des stimuli, qui perturbe la perception du trait à évaluer. 


\section{Question 3 : prononciation à enseigner dans les cours de français langue étrangère (FLE)}

Quant à la question sur la prononciation-cible en FLE, elle confirme dans le cas

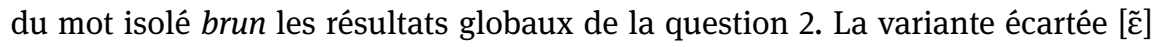
est en effet majoritairement préférée par les informateurs : 53,13\% (51/96) d'entre eux la choisissent, contre 30,21\% (29/96) pour la variante arrondie [õ]. Cette différence est hautement significative à $p<0,001$ (cf. figure 138).

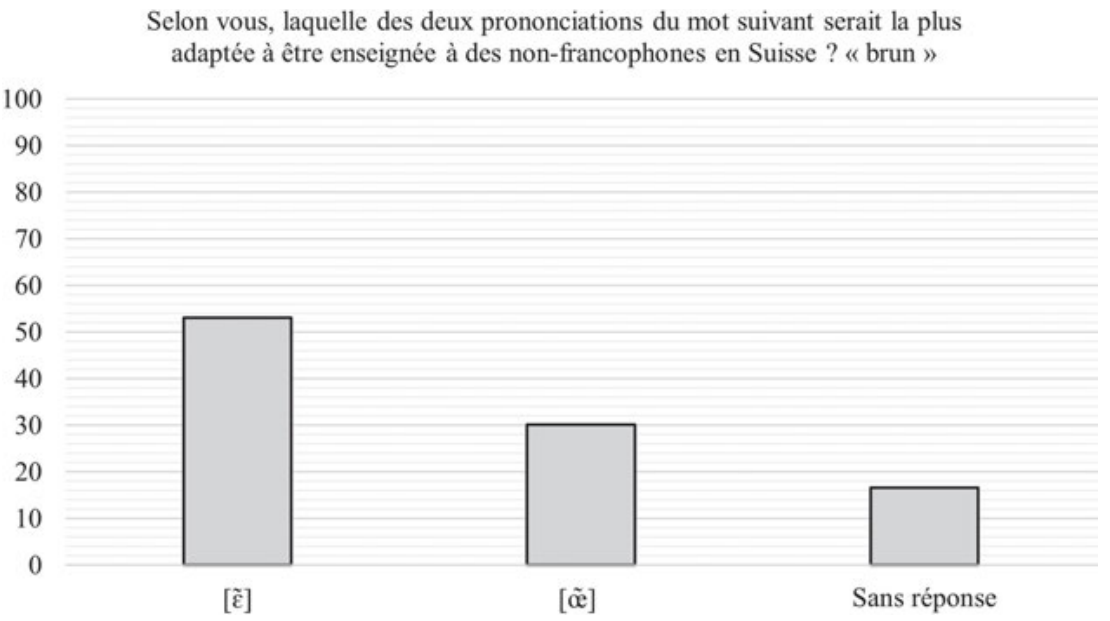

Figure 138 : Voyelle privilégiée (en \%) dans le mot <brun> pour les cours de français langue étrangère (FLE) $(\mathrm{n}=96)$ - Mots isolés ( $p<0,001$, coef. $=1,78$, e.s. $=0,34, t=5,23)$.

Contrairement aux résultats obtenus pour la question 2, le résultat du déterminant un dans le syntagme d'un autre côté est ici similaire à celui du mot isolé brun.

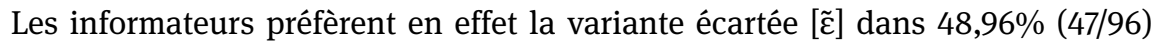
des cas, contre 38,54\% (37/96) pour la variante arrondie [õ]], la différence étant significative à $p<0,05$ (cf. figure 139).

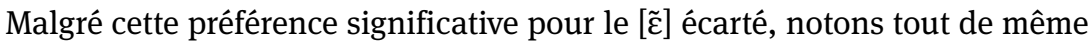
que le niveau $(p<0,05)$ est ici considérablement plus faible que dans le cas du mot isolé brun. Il semble donc à nouveau que l'effet de longueur du syntagme ait quelque peu perturbé les informateurs dans leur perception du trait à évaluer, ce qui plaiderait à nouveau pour un faible degré de saillance de l'opposition. Les résultats touchant au syntagme d'un autre côté doivent donc, ici comme dans les deux autres questions, être interprétés avec précaution.

En conclusion, dans les perceptions des informateurs suisses romands, contrairement à ce qui était suggéré par l'analyse des productions (cf. 5.2.2), la 
Selon vous, laquelle des deux prononciations du groupe de mots suivant serait la plus adaptée à être enseignée à des non-francophones en Suisse ? « D'un autre côté ”

100
90
80
70
60
50
40
30
20
10
0

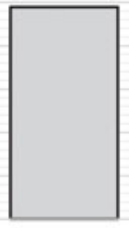

$[\tilde{\varepsilon}]$

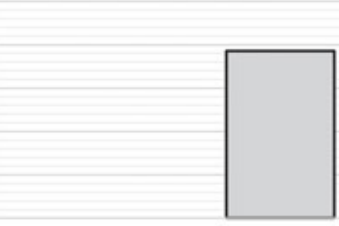

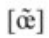

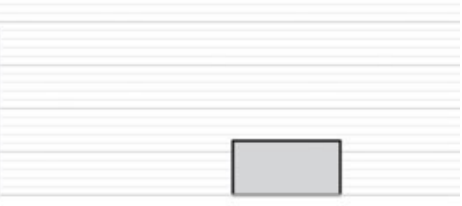

Sans réponse

Figure 139 : Voyelle privilégiée (en \%) dans le mot <un> pour les cours de français langue étrangère (FLE) $(\mathrm{n}=96)$ - Texte $\operatorname{PFC}(p<0,05$, coef. $=0,17$, e.s. $=0,8, t=2,40)$.

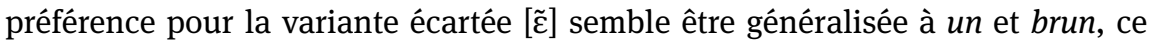
dernier ne montrant donc pas plus de résistance à la neutralisation que le mot un. Dès lors, si les productions suggéraient un maintien de l'opposition dans les productions de l'opposition brun brin chez les locuteurs-modèles, les résultats suggèrent ici plutôt une neutralisation généralisée, ce qui incite à ne pas l'inclure dans la définition de la norme de prononciation suisse romande. À ce jour, étant donnés ces résultats des productions et des perceptions, ce trait ne peut donc pas être pris en compte dans cette définition et devra faire l'objet d'études supplémentaires pour montrer dans quelle mesure la tendance est à la neutralisation du trait ou à son maintien.

Soulignons finalement un constat secondaire des analyses : les résultats montrent une différence générationnelle dans l'évaluation des deux variantes, puisqu'en moyenne, dans les deux contextes analysés, les informateurs de moins

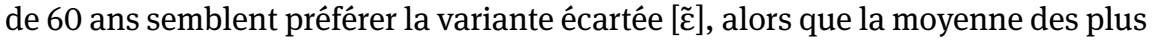
de 60 ans semble au contraire privilégier la variante arrondie [õ̃]. Ceci confirme (1) les résultats de Pooley $(2012,128)$, qui observait, entre autres, une tendance des présentateurs de télévision âgés à la conservation de l'opposition, et (2), en partie, les résultats d'Andreassen/Maître/Racine (2010, 223-224), qui montraient, pour des locuteurs non professionnels, une neutralisation de l'opposition chez les jeunes Genevois (neutralisation aboutie) et les jeunes Neuchâtelois (neutralisation en cours). Notons cependant que la dimension géographique mentionnée par les auteurs (Genevois vs. Neuchâtelois), bien que testée dans mon étude (cf. 3.3.2 : tableau 11, figure 5), n’a pas donné de résultats significatifs. 


\subsubsection{Discussion}

Dans le cadre des tests de perception présentés dans ce sous-chapitre, les hypothèses formulées quant à l'appartenance à la norme endogène suisse romande de cinq traits de prononciation analysés dans les productions de locuteurs-modèles de cette région (cf. 5.2.1) ont pu être testées sur la base d'évaluations perceptives de 96 informateurs suisses romands sélectionnés selon un échantillonnage par quotas. Les principaux résultats de ces tests de perception sont résumés dans le tableau 75, dans lequel ils sont également comparés aux résultats des productions. Précisons qu'étant donnée l'instabilité de la norme suisse romande et les changements en cours, les tendances actuelles ont également été prises en compte dans l'aperçu.

Tableau 75 : Comparaison des résultats touchant aux productions et perceptions pour les cinq traits étudiés dans le cas de la Suisse romande.

\begin{tabular}{|c|c|c|c|c|c|c|}
\hline \multirow{2}{*}{$\begin{array}{l}\text { Traits de } \\
\text { prononciation } \\
\text { suisses romands }\end{array}$} & \multirow{2}{*}{$\begin{array}{l}\text { Contextes } \\
\text { testés }\end{array}$} & \multicolumn{2}{|c|}{ Productions } & \multicolumn{2}{|c|}{ Perceptions } & \multirow[t]{2}{*}{ Commentaires } \\
\hline & & $\begin{array}{l}\text { Norme } \\
\text { actuelle }\end{array}$ & $\begin{array}{l}\text { Tendance } \\
\text { actuelle }\end{array}$ & $\begin{array}{l}\text { Norme } \\
\text { actuelle }\end{array}$ & $\begin{array}{l}\text { Tendance } \\
\text { actuelle }\end{array}$ & \\
\hline $\begin{array}{l}\text { 1. Opposition } \\
\text { /a/:/a:/ en } \\
\text { syllabe finale } \\
\text { fermée }\end{array}$ & $\begin{array}{l}\text { patte /pat/ } \\
\sim \text { pâte / } \\
\text { pa:t/ / } \\
\text { pa:t/ }\end{array}$ & /a/: /a:/ & $\begin{array}{l}-|a|:|a:| \\
-|a|\end{array}$ & /a/: /a:/ & $(/ a /)$ & $\begin{array}{l}\text { Perceptions : } \\
\text { tendance } \\
\text { au /a/ } \\
\text { uniquement } \\
\text { dans la } \\
\text { première } \\
\text { question } \\
\text { Perceptions : } \\
\text { trait peu } \\
\text { saillant }\end{array}$ \\
\hline $\begin{array}{ll}2 . & \text { Opposition } \\
\text { /e/ : /e:/ en } \\
\text { syllabe finale } \\
\text { ouverte (+ } \\
\text { diphtongaison) }\end{array}$ & $\begin{array}{l}\text { penser / } \\
\text { pãse/ } \\
\text { pensée / } \\
\text { pãse:/ ( / } \\
\text { pãse:'/) }\end{array}$ & /e/: /e:/ & /e/ & /e/: /e:/ & I & $\begin{array}{l}\text { Perceptions: } \\
\text { diphtongaison } \\
\text { /pãse:j/ non } \\
\text { testée car } \\
\text { produite par } \\
\text { aucun locuteur }\end{array}$ \\
\hline $\begin{array}{l}\text { 3. Opposition } \\
/ \mathrm{e} /: / \varepsilon / \\
\text { en syllabe } \\
\text { finale ouverte } \\
\text { (distinction de } \\
\text { certains temps } \\
\text { et modes) }\end{array}$ & $\begin{array}{l}\text { je pourrai } \\
\text { /e/ je } \\
\text { pourrais } \\
\mid \varepsilon /\end{array}$ & $\mid \mathrm{e} /: / \varepsilon /$ & $|\varepsilon|$ & $\mid \mathrm{e} /: / \varepsilon /$ & $\mid \varepsilon /$ & 1 \\
\hline
\end{tabular}


Tableau 75 (suite)

\begin{tabular}{|c|c|c|c|c|c|c|}
\hline \multirow{2}{*}{$\begin{array}{l}\text { Traits de } \\
\text { prononciation } \\
\text { suisses romands }\end{array}$} & \multirow{2}{*}{$\begin{array}{l}\text { Contextes } \\
\text { testés }\end{array}$} & \multicolumn{2}{|c|}{ Productions } & \multicolumn{2}{|c|}{ Perceptions } & \multirow[t]{2}{*}{ Commentaires } \\
\hline & & $\begin{array}{l}\text { Norme } \\
\text { actuelle }\end{array}$ & $\begin{array}{l}\text { Tendance } \\
\text { actuelle }\end{array}$ & $\begin{array}{l}\text { Norme } \\
\text { actuelle }\end{array}$ & $\begin{array}{l}\text { Tendance } \\
\text { actuelle }\end{array}$ & \\
\hline $\begin{array}{l}\text { 4. Opposition } \\
/ \tilde{\varepsilon} /: / \tilde{e} /\end{array}$ & 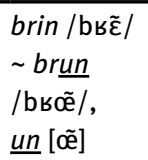 & $\begin{array}{l}\mid \tilde{\varepsilon} /: / \tilde{\Theta} / \\
\mid \tilde{\varepsilon} /\end{array}$ & $\mid \tilde{\varepsilon} /$ & $\mid \tilde{\varepsilon} /$ & I & $\begin{array}{l}\text { Perceptions: } \\
\text { trait peu } \\
\text { saillant }\end{array}$ \\
\hline $\begin{array}{l}\text { 5. Préférence de } \\
\text { la diérèse à la } \\
\text { synérèse }\end{array}$ & 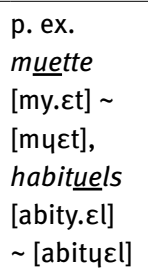 & Synérèse & & Diérèse & 1 & $\begin{array}{l}\text { Perceptions : } \\
\text { trait peu } \\
\text { saillant }\end{array}$ \\
\hline
\end{tabular}

Comme le montre le tableau 75, les résultats touchant à la norme de Suisse romande sont particulièrement complexes. Il semble en effet que cette norme se trouve à ce jour dans une phase de changement : plusieurs traits actuellement majoritaires présentent des tendances allant en direction du français de référence, et les productions et perceptions n'affichent pas toujours les mêmes tendances. Cependant, une réduction schématique de cette complexité aux résultats les plus essentiels montre que, parmi les traits étudiés, trois peuvent être considérés comme faisant partie de la norme de prononciation suisse romande, alors que deux n'en font $a$ priori pas partie, ces derniers devant encore faire l'objet d'analyses de production et de perception plus poussées. Ce résumé schématique est présenté dans le tableau 76, dans lequel (1) les traits sont classés selon leur appartenance (ou non) à la norme de prononciation suisse romande, (2) les contextes testés sont énumérés, (3) les tendances s'éloignant de la norme actuelle sont spécifiées et (4) la non-prise en compte des deux derniers traits est justifiée d'après les résultats obtenus (cf. tableau 76 ).

Étant donnée la complexité de ces résultats, quelques commentaires additionnels s'imposent. Tout d'abord, si les productions et perceptions montrent parallèlement qu'au moins trois traits saillants du français parlé en Suisse romande sont privilégiés dans des contextes liés à la norme, l'on remarquera également que cette norme tend dans de nombreux cas vers la norme du français de référence. Si norme endogène il y a, cette norme n'est donc pas stable et elle se rapproche fortement de la norme de référence, ce qui ne concorde pas avec la distance linguistique par rapport à la norme « du centre » que sous-entend une norme endogène. Les percep- 
Tableau 76 : Résumé des traits appartenant (ou non) à la norme de prononciation suisse romande.

\begin{tabular}{|c|c|c|}
\hline \multicolumn{3}{|c|}{ Traits faisant partie de la norme de prononciation suisse romande } \\
\hline Trait & Contextes testés & Tendance \\
\hline $\begin{array}{l}\text { L'opposition de timbre et de } \\
\text { durée /a/: /a: en syllabe } \\
\text { finale fermée }\end{array}$ & patte /pat/ pâte /pa:t/ & $\begin{array}{l}|\mathrm{a} /:| \mathrm{a}: />|\mathrm{a} /:| \mathrm{a}: / \\
>/ \mathrm{a} /\end{array}$ \\
\hline $\begin{array}{l}\text { Opposition de durée /e/: } \\
\text { /e:/ en syllabe finale ouverte }\end{array}$ & $\begin{array}{l}\text { penser /pãse/ pensée } \\
\text { /pãse:/ }\end{array}$ & $(/ \mathrm{e} /: / \mathrm{e} />/ \mathrm{e} /)$ \\
\hline $\begin{array}{l}\text { Opposition } / \mathrm{e} /: / \varepsilon / \text { en } \\
\text { syllabe finale ouverte }\end{array}$ & je pourrai /e/ je pourrais / $/ \varepsilon /$ & $(/ \mathrm{e} /: / \varepsilon />/ \varepsilon /)$ \\
\hline \multicolumn{3}{|c|}{ Traits ne faisant $a$ priori pas partie de la norme de prononciation suisse romande } \\
\hline Trait & Contextes testés & Justification \\
\hline Opposition / $\tilde{\varepsilon} /: / \tilde{e} /$ & 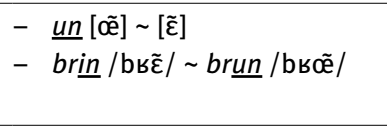 & $\begin{array}{ll}\text { - } & \text { un neutralisé en } / \tilde{\varepsilon} / \\
\text { - } & \text { brun : productions et } \\
& \text { perceptions contradictoires }\end{array}$ \\
\hline Préférence pour la diérèse & 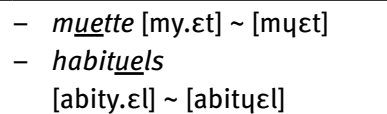 & $\begin{array}{l}\text { - Productions et perceptions } \\
\text { contradictoires }\end{array}$ \\
\hline
\end{tabular}

tions, en combinaison aux productions et représentations et aux attitudes, montrent ainsi à nouveau qu'il est difficile d'envisager l'existence d'une norme de prononciation romande stable présentant un prestige manifeste.

Notons finalement que le test de perception effectué pour la Suisse romande doit, lui aussi, être soumis à quelques réserves, similaires aux cas de Paris et du Québec et donc résumées dans le sous-chapitre 6.4 (cf. infra).

\subsection{Québec}

En ce qui concerne le Québec, les tests de perception touchent aux neuf traits vocaliques dont les réalisations ont été analysées dans l'usage des locuteursmodèles de Radio-Canada dans le chapitre sur les productions (cf. 5.3). Ces traits correspondent à ceux étudiés dans les principales études ayant abordé la norme de prononciation québécoise (cf. Cox 1998 ; Reinke 2004 ; 2005 ; Ostiguy/Tousignant 2008 ; Bigot/Papen 2013). Les six premiers traits concernent des voyelles orales : l'opposition de timbre et de durée /a/ : /a:/ (cf. 6.3.1), la réalisation du /a/ final dans <-oi> en tant que [wa] ou [wa] (cf. 6.3.2), l'opposition de durée $/ \varepsilon /$ : $/ \varepsilon: /$ et la diphtongaison $\mathrm{du} / \varepsilon: /$ en $\left[\mathrm{a}^{3}\right]$ réunies en une double paire de voyelles $/ \varepsilon /$ : $/ \varepsilon: / \sim / a^{3} /$ (cf. 6.3.3), l'ouverture et le relâchement des voyelles /i, y, u/ (cf. 6.3.4) 
ainsi que leur dévoisement ou effacement potentiel (cf. 6.3.5). Les trois derniers traits sont pour leur part relatifs à des voyelles nasales : l’opposition /õ̃/ : / $\tilde{\varepsilon} /$ (cf. 6.3.6) ainsi que les réalisation phonétiques de /ã/ (cf. 6.3.7) et de / $\tilde{\varepsilon} /$ (cf. 6.3.8).

\subsubsection{Opposition /a/ : / $a$ /}

Parallèlement à Paris et à la Suisse romande, le premier trait à avoir été testé dans le cas du Québec est l'opposition /a/ : /a:/. L'analyse des productions a en effet montré que les locuteurs-modèles ont tendance, comme en français québécois vernaculaire, à réaliser quasiment systématiquement non pas une simple opposition de timbre, mais également une opposition de durée (cf. 5.3.1.1).

\section{Question 1 : évaluation (sans contexte explicite)}

En ce qui concerne le mot isolé pâte, les résultats de la question qui visait à faire évaluer l'opposition sur la base d'une échelle (0\%-100\%) et sans mention explicite du contexte indiquent que la variante postérieure allongée [a:] est nettement mieux évaluée que la variante antérieure brève [a] : le [a:] obtient en effet un score moyen de 92,19\% (écart-type : 11,76\%), contre seulement 46,16\% (écart-type : 28,85\%) pour le [a], la différence entre les deux scores moyens étant hautement significative à $p<0,001$ (cf. figure 140).

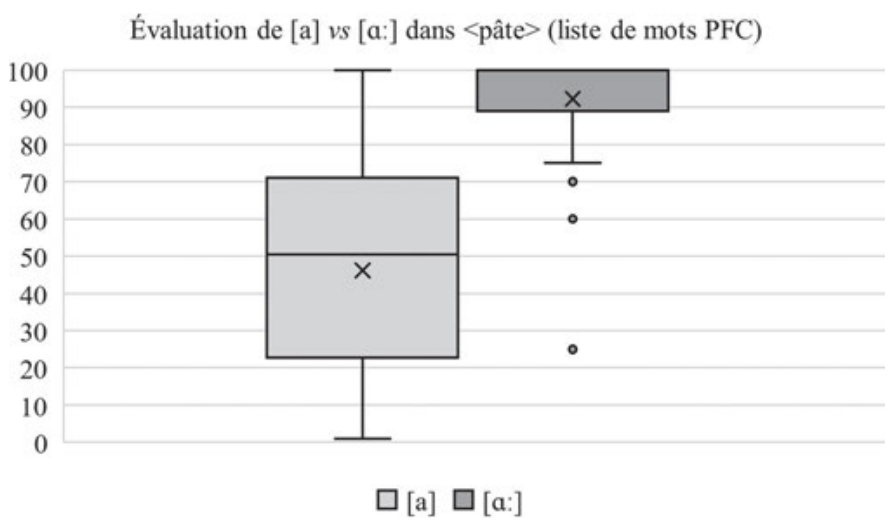

Figure 140 : Évaluation perceptive (en \%) des voyelles [a] et [a:] par les informateurs québécois $(\mathrm{n}=96)$ dans le mot isolé <pâte $>(A N O V A: p<0,001)$.

Appliquée dans un deuxième temps au syntagme et plus récemment, son usine de pâtes italiennes, cette même question révèle également une légère préférence 
pour le [a:] postérieur allongé, cette variante obtenant un score moyen de $91,30 \%$ (écart-type : 14,21\%), contre 87,89\% (écart-type : 16,31\%) pour la variante [a]. Selon l'analyse de variance, cette différence n'est cependant pas significative $(p>0,05)$ (cf. figure 141).

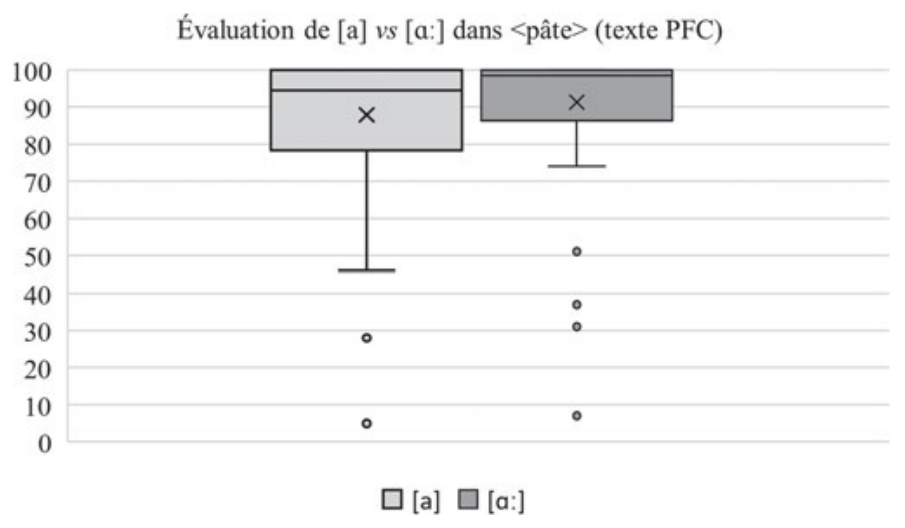

Figure 141 : Évaluation perceptive (en \%) des voyelles [a] et [a:] par les informateurs québécois $(n=96)$ dans le mot <pâte> tiré du texte PFC (ANOVA : $p>0,05)$.

Comme l'ont montré les résultats touchant à Paris et à la Suisse romande, il semble que l'explication de l'écart entre les résultats du mot isolé pâte et du syntagme et plus récemment, son usine de pâtes italiennes doive être cherchée dans la saillance de l'opposition et la différence qui en découle dans chaque cas dans le comportement évaluatif des informateurs, le mot isolé activant plutôt des représentations et le syntagme de véritables perceptions (cf. 3.1). Pour les informateurs, l'évaluation du syntagme est en effet manifestement plus difficile, probablement en raison de sa longueur et de la difficulté qui en résulte à percevoir le trait à évaluer. Cette interprétation est d'autant plus vraisemblable que, dans cette première question, au contraire des questions 2 et 3 , les stimuli ne sont pas comparés directement au sein d'une unique question, mais évalués séparément. C'est donc principalement dans l'opposition claire des mots isolés (qui fait appel à des représentations) que le trait est reconnu, un contexte plus large (qui fait plus appel à des perceptions) faisant disparaître l'opposition dans les perceptions. Dès lors, si la tendance interprétative globale est, comme dans le cas de la Suisse romande, de privilégier le [a:] postérieur allongé, il est important de souligner que le degré de saillance de l'opposition semble relativement faible dans les perceptions. 


\section{Question 2 : prononciation idéale des présentateurs de journaux télévisés}

En ce qui concerne la deuxième question, qui porte sur la prononciation perçue comme étant idéale pour les présentateurs québécois et dans laquelle les deux variantes sont comparées directement (tout comme dans la question 3), les résultats montrent par rapport au mot isolé pâte la même tendance à préférer le [a:] postérieur allongé, cette tendance étant même légèrement plus marquée : 96,88\% (93/96) des informateurs privilégient en effet cette variante, contre seulement 3,13\% (3/96) pour le [a] antérieur bref. Sans surprise, étant donnée la clarté des résultats, le modèle de régression logistique indique que cette différence de taux est hautement significative, à $p<0,001$, alors qu'aucune des variables sociodémographiques ne montre d'effet significatif sur ce résultat ( $p>0,05)$ (cf. figure 142).

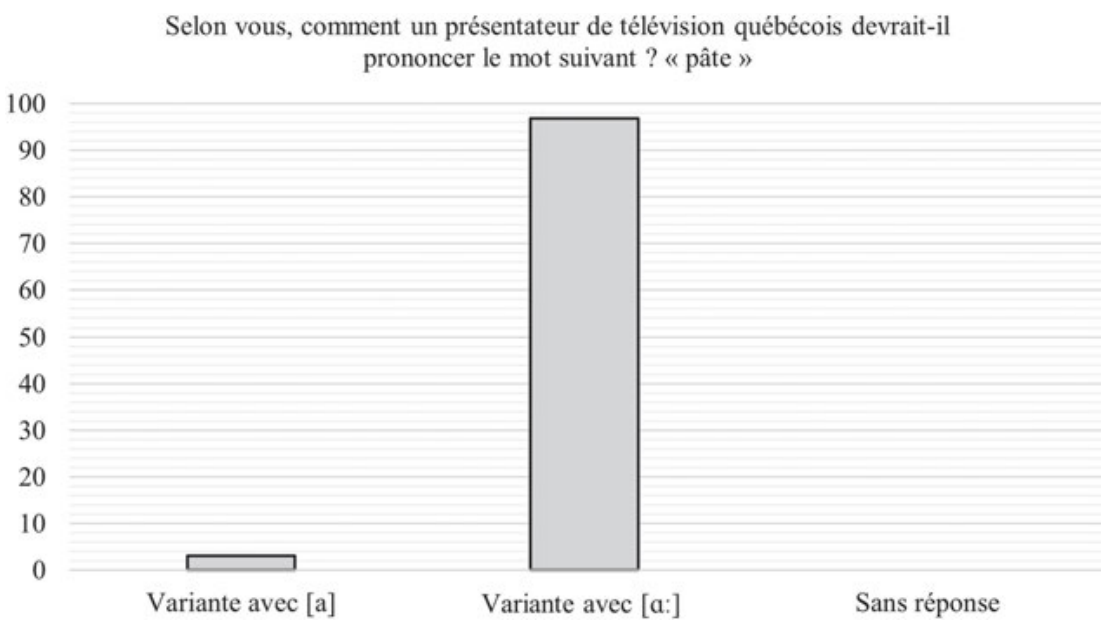

Figure 142 : Voyelle privilégiée (en \%) dans le mot <pâte> pour les présentations de journaux télévisés $(\mathrm{n}=96)$ - Mots isolés $(p<0,001$, coef. $=2,03$, e.s. $=0,07, t=27,85)$.

Les résultats obtenus par le syntagme montrent, pour leur part, une tendance similaire à privilégier le [a:] postérieur allongé, moins forte cependant que dans le cas du mot isolé : les informateurs préfèrent en effet dans 60,42\% (58/96) des cas le [a:], contre 36,46\% (35/96) pour le [a], la différence étant significative à $p<0,001$. Il est intéressant de remarquer que d'après le modèle de régression logistique, il existe parmi les variables sociodémographiques prises en compte un léger effet significatif de l'éducation sur les résultats $(p<0,05)$ : en moyenne, les informateurs ayant un niveau plus élevé, titulaires soit d'un diplôme universitaire, soit d'un diplôme d'études collégiales (DEC) des programmes d'études pré-universitaires, ont ainsi tendance à choisir légèrement plus souvent la variante antérieure brève 
[a] (43,75\%, 21/48) que les informateurs dont le niveau d'éducation moins élevé, titulaires d'un diplôme du primaire, du secondaire, d'une école de métiers ou d'un diplôme d'études collégiales (DEC) des programmes d'études techniques (29,17\%, 14/48) (cf. figure 143).

Selon vous, comment un présentateur de télévision québécois devrait-il prononcer le groupe de mots suivant ? « Et plus récemment son usine de pâtes italiennes »

\section{0 \\ 90 \\ 80 \\ 70 \\ 60 \\ 50 \\ 40 \\ 30 \\ 20 \\ 10 \\ 0}

Primaire, secondaire, école des Université, Cégep général métiers, Cégep professionnel

\section{口Variante avec [a]}

口Variante avec [a:]

- Sans réponse

Figure 143 : Voyelle privilégiée (en \%) dans le mot <pâte> pour les présentations de journaux télévisés $(\mathrm{n}=96)$ - Texte PFC; réponses présentées dans leur globalité $(p<0,001$, coef. $=1,44$, e.s. $=0,29, t=4,92)$ ainsi que selon le niveau d'éducation des informateurs $(p<0,05$, coef. $=$ 0,24 , e.s. $=0,12, t=2,09$ ).

Il est probable que ce [a] antérieur bref, préféré un peu plus souvent par les informateurs plus diplômés, soit attribué par ceux-ci à la variété dominante du « français parisien » (ou « français européen », selon certains informateurs; cf. 4.3.5).

Il existe donc ici une différence fondamentale par rapport aux observations faites dans le cas de Paris : en effet, alors qu'à Paris, les informateurs les plus éduqués préfèrent à plusieurs reprises la norme traditionnelle, tandis que les informateurs les moins diplômés sont plus ouverts au changement (cf. notamment 6.1.2), au Québec, les plus diplômés optent plus souvent pour la norme parisienne que les informateurs moins éduqués, qui eux, privilégient très largement la norme locale. Cette différence s'explique à nouveau par la prise en considération de deux types de changement : alors que dans le cas de Paris, le changement $/ \mathrm{e} /: \mid \varepsilon />/ \mathrm{e} /$ est interne, au Québec, la tendance des locuteurs ayant un niveau d'éducation élevé à préférer plus souvent la généralisation d'une variante parisienne [a] dans l'opposition /a/ : /a:/ n'est pas le produit d'un changement 
interne, mais d'un contact avec la norme parisienne. Soulignons cependant que ces résultats doivent être interprétés avec précaution, étant donné que dans l'ensemble, les résultats des évaluations perceptives de syntagmes sont peu évidents.

\section{Question 3 : prononciation à enseigner dans les cours de français langue étrangère (FLE)}

Les résultats de la troisième question, qui porte sur la prononciation à privilégier dans les cours de FLE, montrent la même tendance que les deux autres questions en ce qui concerne le mot isolé pâte : 97,92\% (94/96) des informateurs préfèrent en effet la variante postérieure allongée [a:], contre seulement deux informateurs $(2,08 \%, 2 / 96)$ qui préfèrent la variante antérieure brève [a]. Sans surprise, étant donnée l'absence d'ambiguïté de ces résultats, la différence entre ces deux taux est hautement significative à $p<0,001$ (cf. figure 144).

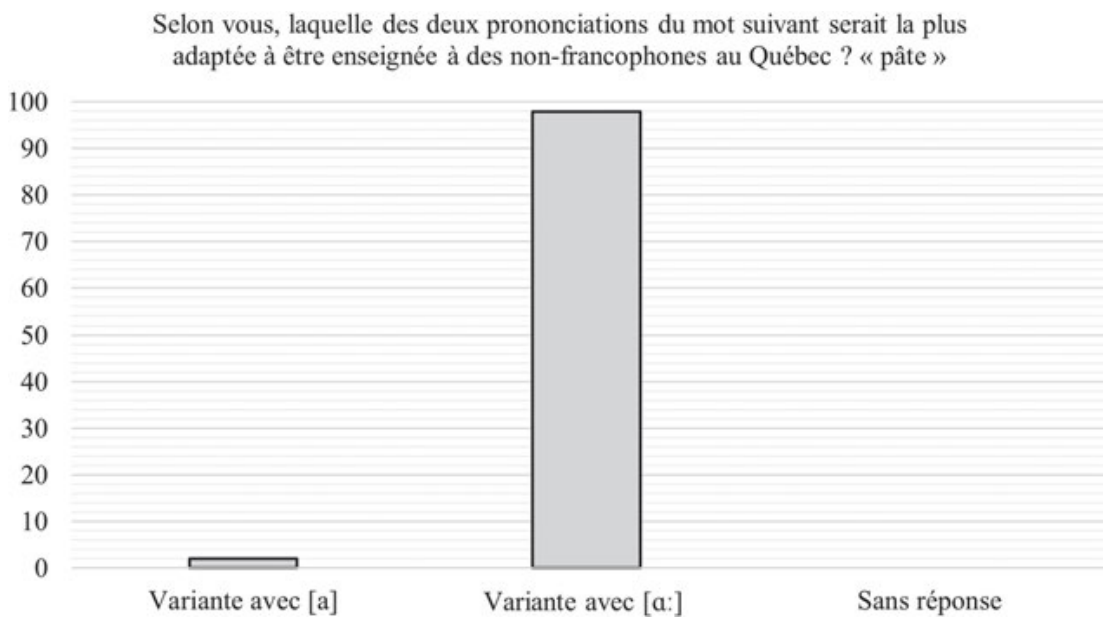

Figure 144 : Voyelle privilégiée (en \%) dans le mot <pâte> pour les cours de français langue étrangère (FLE) $(\mathrm{n}=96)$ - Mots isolés ( $p<0,001$, coef. $=2,07$, e.s. $=0,06, t=34,92)$.

Cette même question posée par rapport au syntagme et plus récemment, son usine de pâtes italiennes produit tout d'abord des résultats confirmant cette tendance : une majorité de $57,29 \%(54 / 96)$ des informateurs préfère la variante avec [a:] postérieur allongé, alors que 38,54\% (36/96) d'entre eux choisissent la variante avec [a] antérieur bref. Par ailleurs, le modèle de régression indique un effet significatif du sexe $(p<0,05)$. Les hommes ont en effet légèrement plus souvent tendance à choisir la variante [a:] postérieure et allongée que les femmes. Ces dernières optent, certes, également en majorité pour cette variante " québécoise », mais 
un pourcentage significativement plus élevé que chez les hommes se prononce en faveur de la variante antérieure brève [a] (femmes : 43,75\%, 21/48; hommes : $33,33 \%, 16 / 48$ ) (cf. figure 145).

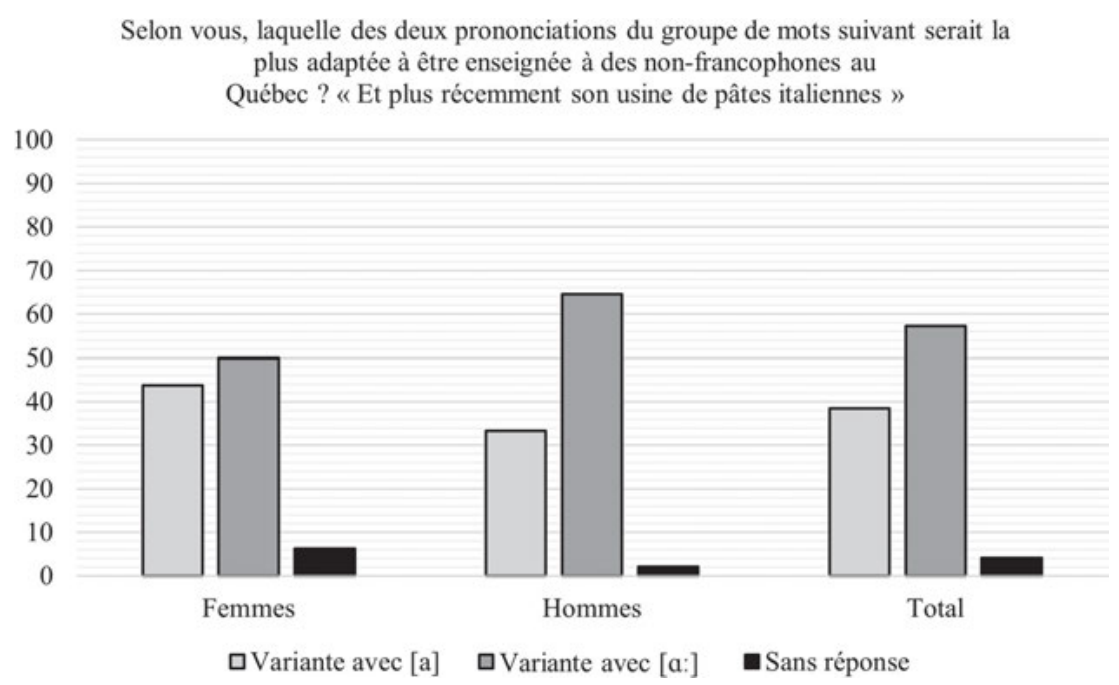

Figure 145 : Voyelle privilégiée (en \%) dans le mot <pâte> pour les cours de français langue étrangère (FLE) $(\mathrm{n}=96)$ - Texte PFC; réponses présentées dans leur globalité $(p<0,001$, coef. $=1,69$, e.s. $=0,27, t=6,23)$ ainsi que selon le sexe des informateurs $(p<0,05$, coef. $=0,25$, e.s. $=0,11, t=2,35$ ).

En supposant que la variante [a] soit globalement associée à la variété dominante $\mathrm{du}$ « français parisien » (ou « français européen », cf. supra) et la variante [a:] aux variétés québécoises, notons que cette préférence des hommes pour le [a:] postérieur allongé n'étonne guère, plusieurs études ayant déjà montré que les hommes ont davantage tendance à utiliser des traits régionaux présentant un certain prestige latent (cf. entre autres Labov 1963 ; Rovere 1982 ; Bourdieu 1982 ; cf. 2.1.2.2). En outre, le questionnaire sur les représentations et attitudes a révélé dans l'ensemble chez les femmes une plus grande tendance à l'insécurité linguistique et à un usage proche du standard (cf. 4.2.2), ce qui expliquerait leur choix plus fréquent de la variante " parisienne » [a]. Les réserves envers les résultats portant sur les évaluations du syntagme dans les deux autres questions sont cependant également valables ici, raison pour laquelle ces résultats doivent être interprétés avec précaution.

En conclusion, les résultats ont permis de montrer que le maintien de l'opposition /a/ : /a:/ observé dans l'analyse des productions (cf. 5.3.1.1) se confirme très largement dans les évaluations perceptives des informateurs québécois : à 
tout le moins dans le mot pâte, le [a:] allongé postérieur est non seulement utilisé par une quasi-totalité des informateurs, mais également très largement préféré dans les perceptions de locuteurs québécois « ordinaires ». Ainsi, sans surprise, l'opposition /a/ : /a: est maintenue et stable au sein de la norme de prononciation québécoise. Relevons également un résultat secondaire découlant des effets significatifs de variables sociodémographiques dans les deux dernières questions : il semble que les informateurs plus diplômés (cf. figure 143) et les informatrices (cf. figure 145) présentent une tendance légèrement plus élevée à opter pour la variante [a] que les non-diplômés et les locuteurs masculins. Cette tendance est cependant relativement négligeable, étant donné que ces deux mêmes groupes privilégient tout de même majoritairement la variante /a:/. Il semblerait donc plutôt qu'on puisse relever une plus grande tendance à l'insécurité linguistique dans ces deux groupes sociodémographiques, tendance peu surprenante au vu des résultats de nombreux travaux sociolinguistiques antérieurs (cf. p. ex. Trudgill 1972 ; Owens/Baker 1984 ; Labov 1990 ; 1998 concernant le facteur du sexe ; cf. p. ex. Francard 1989 ; Ledegen 1998 ; L'Eplattenier-Saugy 2002 concernant celui du niveau d'éducation).

\subsection{2 /a/ final dans <-oi> : [wa] [wa]}

Le deuxième trait testé dans le cadre du test de perception effectué au Québec touche également à la postériorisation du [a], mais dans le cas particulier des graphies <oi > et <-ô̂े, dans lesquelles la prononciation varie fortement entre [wa] et [wa] en français québécois selon les mots et les types de syllabes. Rappelons que l'analyse des productions a montré que dans l'usage des locuteurs-modèles, le [a] postérieur est maintenu en syllabe finale ouverte et en syllabe finale fermée dans les mots baignoire et trois, qui sont également les mots ayant fait l'objet des tests de perception.

\section{Question 1 : évaluation (sans contexte explicite)}

Tout d'abord, les résultats de la première question d'évaluation, effectuée sans mention de contexte et sur la base d'une échelle (0\%-100\%), montrent que pour le mot isolé baignoire, l'évaluation de la variante endogène avec [a] postérieur est significativement meilleure que celle de la variante avec [a] antérieur : la variante postérieure [a] obtient en effet un score de 90,81\% (écart-type : 14,08\%), contre 82,53\% (écart-type : 20,02\%) pour la variante antérieure [a], cette différence étant hautement significative $(p<0,001)$ selon l'analyse de variance (cf. figure 146). 
Évaluation de [a] $v s[a]$ dans < baignoire $>$ (liste de mots PFC)

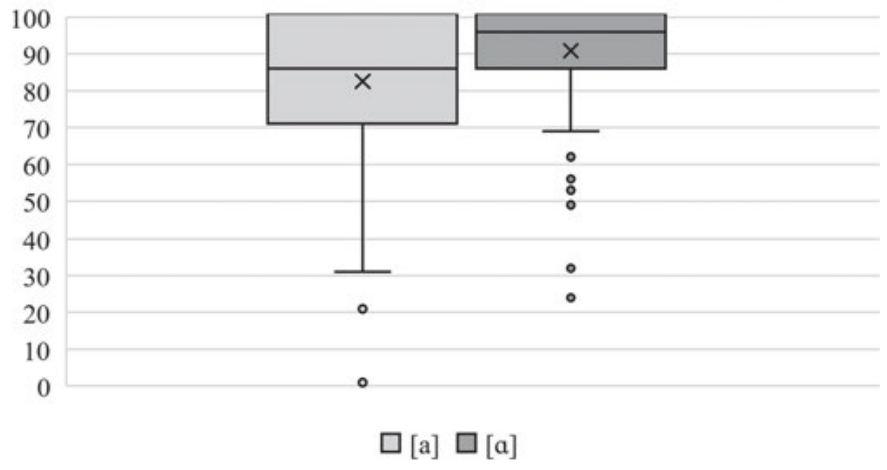

Figure 146 : Évaluation perceptive (en \%) des voyelles [a] et [a] par les informateurs québécois $(\mathrm{n}=96)$ dans le mot isolé <baignoire> (ANOVA : $p<0,001)$.

Comme souvent dans les trois tests de perception analysés dans le cadre de ce chapitre, l'évaluation perceptive du syntagme (ici : trois échevins) engendre des résultats plus complexes. En effet, les deux variantes obtiennent des scores très similaires : celle avec [a] postérieur atteint un score de 87,79\% (écart-type : $15,66 \%$ ), contre $86,84 \%$ (écart-type : 14,78\%) pour la variante avec [a] antérieur, cette différence n'étant pas significative $(p>0,05)$ (cf. figure 147).

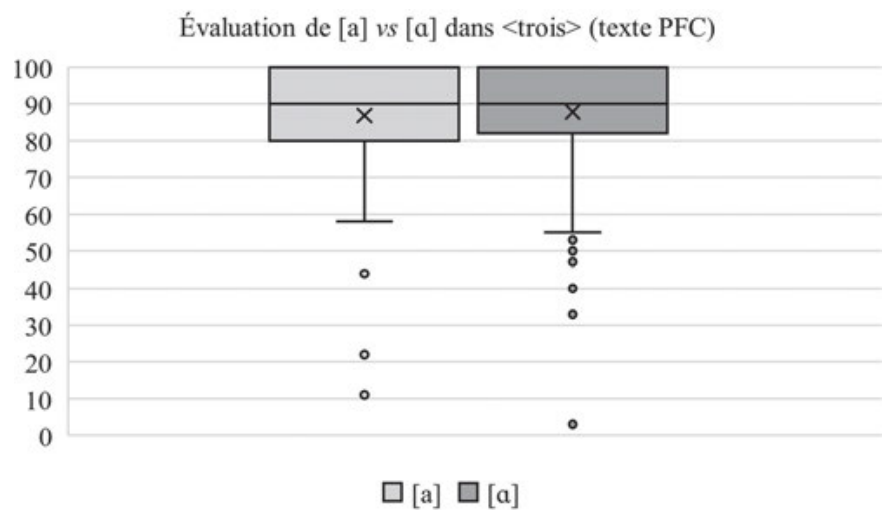

Figure 147 : Évaluation perceptive (en \%) des voyelles [a] et [a] par les informateurs québécois $(\mathrm{n}=96)$ dans le mot $<$ trois> tiré du texte PFC $($ ANOVA $: p>0,05)$.

Le problème méthodologique de la longueur du syntagme combiné au fait que l'évaluation n'a pas été faite dans le cadre d'une comparaison directe des variantes semble donc se poser également dans le cas présent, les informateurs 
présentant manifestement des difficultés à percevoir le trait à évaluer. La fiabilité des résultats touchant au syntagme doit donc, ici aussi, être remise en question. C'est la raison pour laquelle l'interprétation globale se base à nouveau principalement sur les résultats du mot isolé (baignoire), qui montrent ici que les Québécois ont globalement tendance accorder leur préférence au [a] postérieur dans la graphie $<$ oi $>$.

\section{Question 2 : prononciation idéale des présentateurs de journaux télévisés}

Les réponses à la deuxième question, qui porte sur la prononciation perçue comme idéale par les informateurs pour la présentation de journaux télévisés au Québec, montrent également une forte préférence pour la variante endogène postérieure [a] : les informateurs la choisissent en effet dans 72,92\% (70/96) des cas, seuls 22,92\% (22/96) d'entre eux optant pour la variante antérieure [a]. Étant donnée la clarté des résultats, le modèle de régression montre que la différence entre ces taux est hautement significative $(p<0,001)$ (cf. figure 148).

Selon vous, comment un présentateur de télévision québécois devrait-il prononcer le mot suivant ? « baignoire »

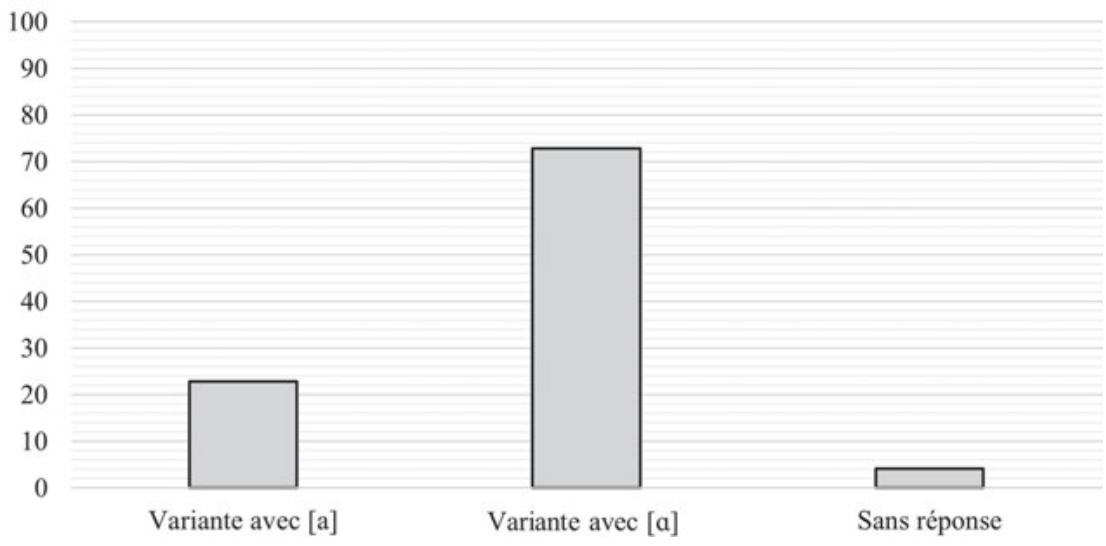

Figure 148 : Voyelle privilégiée (en \%) dans le mot <baignoire> pour les présentations de journaux télévisés $(\mathrm{n}=96)$ - Mots isolés $(p<0,001$, coef. $=1,61$, e.s. $=0,23, t=7,06)$.

Les résultats touchant au syntagme trois échevins présentent des tendances très similaires, bien que moins fortes : globalement, 53,13\% (51/96) des informateurs choisissent le stimulus comportant la variante postérieure [a], et 22,92\% (22/96) celui comportant la variante antérieure [a]. Notons ce faisant le taux relativement élevé de 23,96\% (23/96) d'informateurs n'ayant pas répondu à la question, révélant à nouveau une certaine difficulté à percevoir clairement le trait à évaluer, et 
ce, même dans la présente situation de comparaison directe des deux stimuli. Soulignons par ailleurs également un effet significatif du sexe $(p<0,01)$, les hommes ayant davantage tendance que les femmes à préférer la variante postérieure [a] (cf. figure 149).

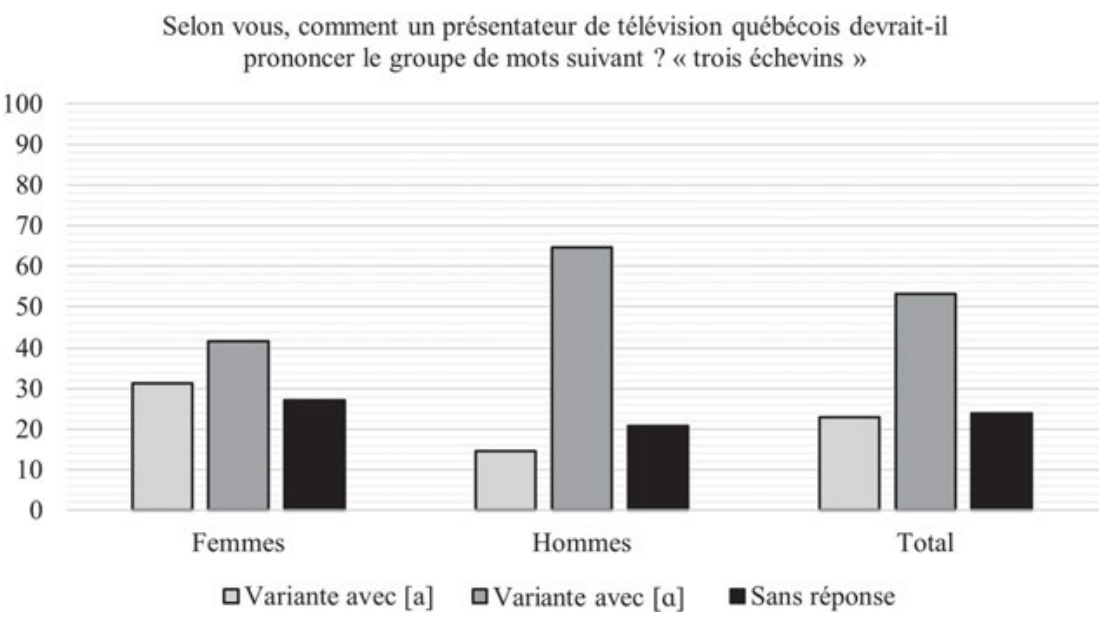

Figure 149 : Voyelle privilégiée (en \%) dans le mot <trois> pour les présentations de journaux télévisés $(\mathrm{n}=96)$ - Texte PFC; réponses présentées dans leur globalité $(p<0,001$, coef. $=2,55$, e.s. $=0,37, t=6,99$ ) ainsi que selon le sexe des informateurs ( $p<0,01$, coef. $=0,44$, e.s. $=0,14$, $t=3,06)$.

De manière similaire à ce qui a déjà pu être observé dans le cas de l'opposition /a/ : /a:/ (cf. 6.3.1), la préférence des informateurs masculins pour la variante postérieure $[a]$ n'est guère surprenante : plusieurs études sociolinguistiques ont en effet montré qu'en moyenne, les hommes ont généralement plus tendance à privilégier les variantes régionales au prestige latent (ici le [a] postérieur) et les femmes plus tendance à l'insécurité linguistique, ce qui expliquerait leur choix plus fréquent de la variante antérieure [a] associée à un français "parisien » ou « européen » (cf. 6.3.1). Ces tendances, bien que significatives, ne remettent cependant pas en cause le résultat global de cette question, étant donné que femmes et hommes préfèrent majoritairement la variante postérieure [a]. Par ailleurs, elles doivent également être interprétées avec précaution en raison du manque de fiabilité des évaluations de syntagmes. 


\section{Question 3 : prononciation à enseigner dans les cours de français langue étrangère (FLE)}

Finalement, les résultats de la dernière question, dans laquelle les informateurs étaient priés de choisir la variante la plus apte à être enseignée dans les cours de FLE, montrent à nouveau une forte tendance en faveur de la variante avec [a] postérieur : en effet, 84,38\% (81/96) des informateurs la privilégient, contre seulement $9,38 \%$ (9/96) pour la variante antérieure [a]. Le modèle de régression indique, sans surprise, que ce taux est hautement significatif $(p<0,001)$ (cf. figure 150).

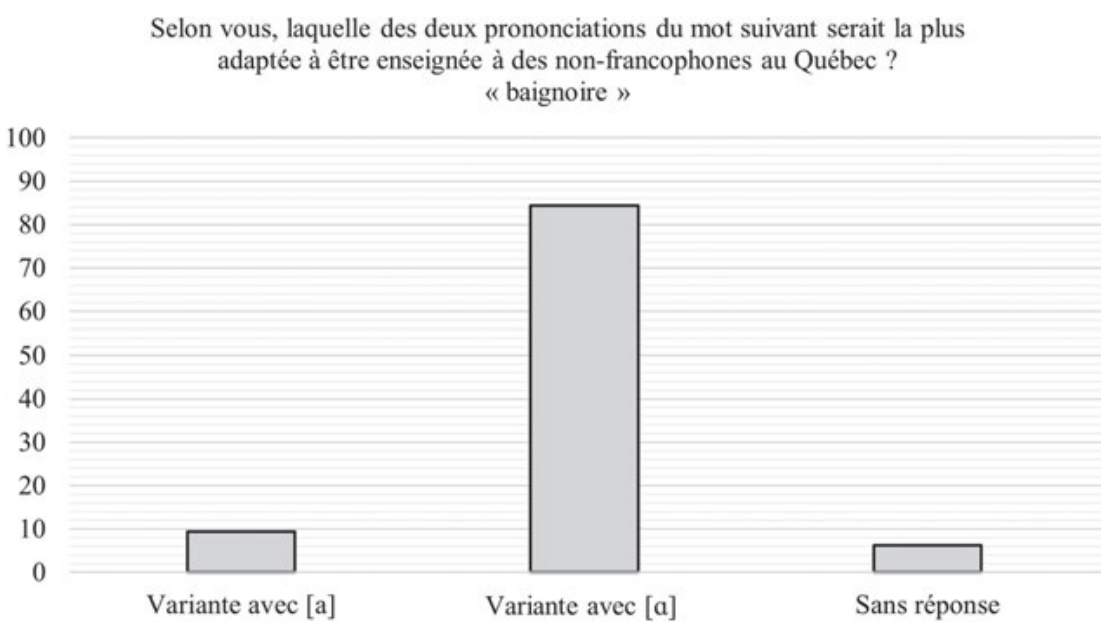

Figure 150 : Voyelle privilégiée (en \%) dans le mot <baignoire> pour les cours de français langue étrangère (FLE) $(\mathrm{n}=96)$ - Mots isolés $(p<0,001$, coef. $=2,05$, e.s. $=0,25 ; t=8,33)$.

Les résultats relatifs au syntagme trois échevins montrent, ici aussi, une tendance à préférer la variante postérieure [a], même si elle s'avère à nouveau légèrement moins forte que dans le cas du mot isolé baignoire. En effet, 56,25\% (54/96) des informateurs choisissent cette variante avec [a] postérieur, alors que 22,92\% (22/96) d'entre eux optent pour le [a] antérieur, cette différence étant hautement significative $(p<0,001)$. Attirons, ici aussi, l'attention sur le nombre considérable de non-réponses $(20,83 \%, 20 / 96)$, qui traduisent à nouveau une difficulté particulière des informateurs à percevoir le trait devant être évalué (cf. figure 151).

Le résultat global confirme donc la préférence fortement majoritaire pour le [a] postérieur chez les informateurs québécois. Cependant, les réserves formulées par rapport à la fiabilité des résultats suscités par ce stimulus syntagmatique sont également valables ici, raison pour laquelle ils doivent être interprétés avec précaution. 
Selon vous, laquelle des deux prononciations du groupe de mots suivant serait la plus adaptée à être enseignée à des non-francophones au Québec? «trois échevins »

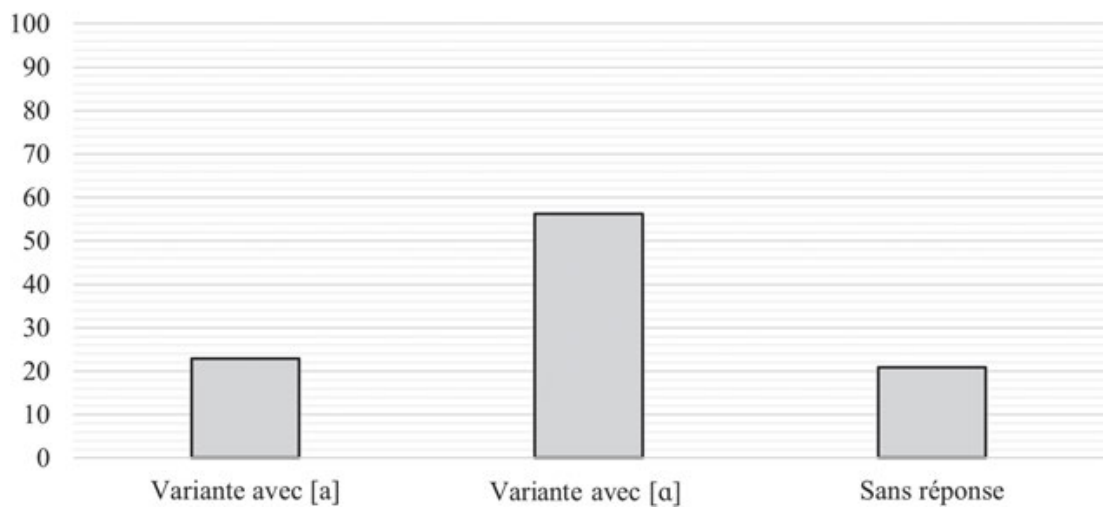

Figure 151 : Voyelle privilégiée (en \%) dans le mot <trois> pour les cours de français langue étrangère (FLE) $(\mathrm{n}=96)$ - Texte PFC $(p<0,001$, coef. $=2,46$, e.s. $=0,34, t=7,19)$.

En conclusion, le maintien du [a] postérieur en syllabe finale ouverte et en syllabe finale fermée dans les mots baignoire et trois mis en lumière dans les productions des locuteurs-modèles (cf. 5.3.1.2) est largement confirmé dans les évaluations perceptives d'informateurs québécois « ordinaires ». Ainsi, le [a] postérieur peut être considéré comme faisant partie intégrante de la norme de prononciation québécoise dans ces deux contextes. Cependant, comme mentionné dans le chapitre sur les productions des locuteurs-modèles (cf. 5.3.1.2), la variation considérable entre les prononciations [wa] et [wa] dans la réalisation du <oi> graphique rend impossible une généralisation de ce résultat à d'autres contextes. Des tests de perception similaires prenant en compte d'autres lexèmes présentant la graphie <oi> devront donc être effectués à l'avenir, notamment dans les mêmes contextes syllabiques, considérés jusqu'ici comme engendrant plutôt la réalisation antérieure [a] (p. ex. boit, moi, noient, reçoit, droit en syllabe finale ouverte ; p. ex. soif, poil, avoine, droite en syllabe finale fermée).

\subsubsection{Opposition $/ \varepsilon /: / \varepsilon: / / \mathrm{a}^{3} /$}

Le cas suivant réunit en une double paire de voyelles $/ \varepsilon /: / \varepsilon: / / \mathrm{a}^{3} /$ deux traits analysés séparément dans le cadre des productions des locuteurs-modèles québécois : l'opposition $/ \varepsilon /: / \varepsilon: /$ et la diphtongaison de la voyelle allongée $/ \varepsilon: /$ dans le mot fête (cf. 5.3.1.3 ; 5.3.1.4). Ce choix est dû à la nécessité de réduire au maximum la durée du test. Rappelons que les analyses de production ont montré 
que l'opposition quantitative $/ \varepsilon /: / \varepsilon$ : / est clairement maintenue dans l'usage des locuteurs-modèles et que la diphtongaison est " admise » lorsqu'elle n'est que " légère » (cf. 5.3.1.4). Ainsi, trois stimuli du mot fête représentant les variantes brève, allongée et légèrement diphtonguée ont été pris en compte dans le test de perception : fête $\left([\mathrm{f} \varepsilon \mathrm{t}] \sim[\mathrm{f} \varepsilon \mathrm{t} t] \sim\left[\mathrm{fa}^{3} \mathrm{t}\right]\right.$. De même que pour les analyses de production, précisons en outre qu'aucune occurrence correspondant à ces deux traits n'a été trouvée dans le texte PFC. Les tests de perception ont ainsi été effectués uniquement sur la base de mots isolés.

\section{Question 1 : évaluation (sans contexte explicite)}

Les analyses touchant à la question d'évaluation des stimuli sur une échelle de scores allant de $0 \%$ à 100\% montrent tout d'abord une large préférence pour la variante allongée [ع:] : elle obtient en effet un score de 93,01\% (écart-type : 8,38\%), contre $79,19 \%$ (écart-type : 22,37\%) pour la variante avec légère diphtongaison [a ${ }^{3}$ ] et 50,01\% (écart-type : 33,49\%) pour la variante brève [ $\varepsilon$ ], ces différences étant toutes deux hautement significatives $(p<0,001)$. On notera par ailleurs avec intérêt que la variante diphtonguée $\left[\mathrm{a}^{3}\right]$ obtient un résultat significativement supérieur à la variante brève $[\varepsilon](p<0,001)$ (cf. figure 152).

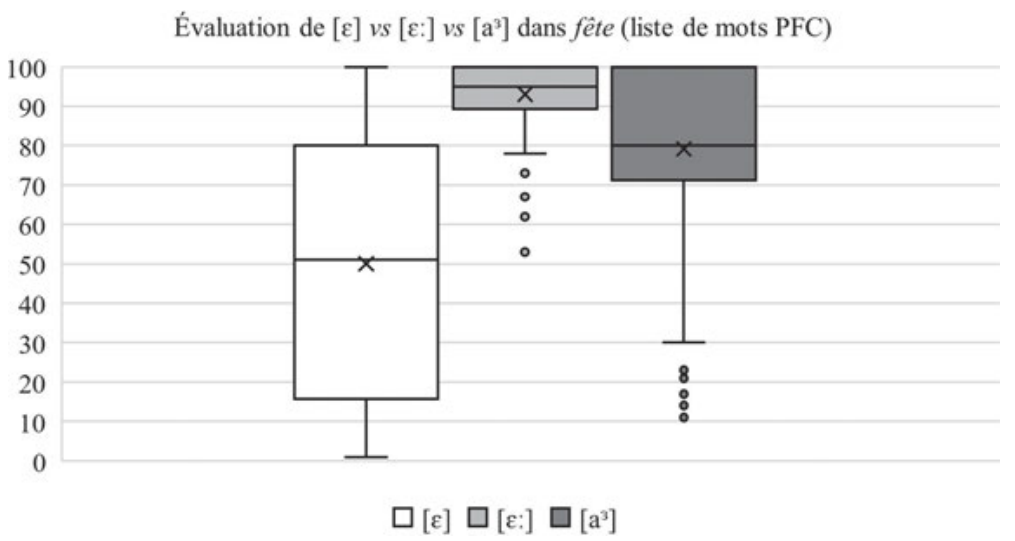

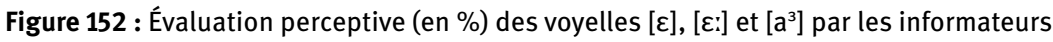
québécois $(\mathrm{n}=96)$ dans le mot isolé <fête $>\left([\varepsilon]\right.$ vs. $[\varepsilon]: p<0,001 ;[\varepsilon:]$ vs. $\left[\mathrm{a}^{3}\right]: p<0,001 ;\left[\mathrm{a}^{3}\right]$ vs. $[\varepsilon]: p<0,001)$.

Il semble donc exister une hiérarchie normative très claire entre les trois variantes, le [ $\varepsilon^{\text {: }}$ étant le plus acceptable, devant le $\left[\mathrm{a}^{3}\right]$ légèrement diphtongué et le $[\varepsilon]$ bref. Soulignons également l'écart-type considérable dans l'évaluation de cette der- 
nière variante (33,49\%), qui montre une grande incertitude dans les réponses des informateurs et confirme donc d'autant plus cette hiérarchie.

Notons finalement que l'analyse de variance révèle aussi un effet significatif du sexe par rapport au stimulus contenant le [ $\left.\varepsilon^{:}\right]$allongé $(F=10,72, p<0,01)$, les femmes évaluant ce stimulus de manière nettement plus positive que les hommes. Il est possible d'y voir un indice d'un prestige relativement élevé de cette variante, étant donnée la préférence moyenne des hommes pour les traits régionaux et des femmes pour les traits standards (cf. entre autres Labov 1963 ; Rovere 1982 ; Bourdieu 1982 ; cf. 2.1.2.2). Mais cette hypothèse devra être confirmée dans les deux questions suivantes, qui comparent plus directement les deux stimuli.

\section{Question 2 : prononciation idéale des présentateurs de journaux télévisés}

Les résultats touchant à la prononciation perçue comme idéale pour les présentateurs de journaux télévisés montrent des tendances très similaires : une vaste majorité de 80,21\% (77/96) des informateurs choisit la variante allongée [ع:], les $19,79 \%$ (19/96) restants préférant la variante légèrement diphtonguée $\left[\mathrm{a}^{3}\right]$ et aucun informateur ne privilégiant le $[\varepsilon]$ bref. Le modèle de régression indique que la différence entre les taux du [ $\left.\varepsilon^{:}\right]$et $d u\left[a^{3}\right]$ est significative $(p<0,001)$ (cf. figure 153).

Selon vous, comment un présentateur de télévision québécois devrait-il prononcer le mot suivant ? « fête »

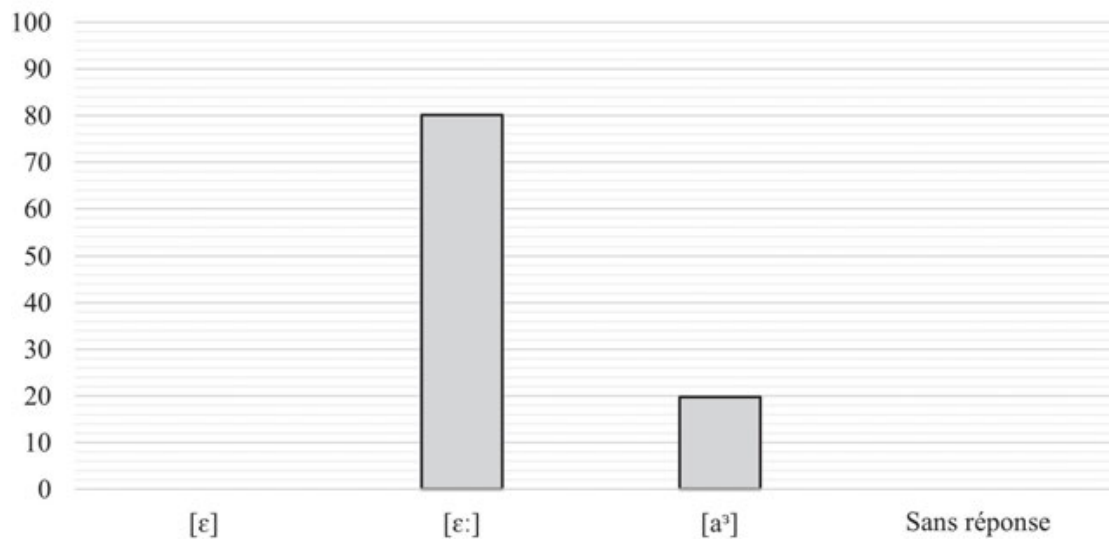

Figure 153 : Voyelle privilégiée (en \%) dans le mot <fête> pour les présentations de journaux télévisés $(\mathrm{n}=96)$ - Mots isolés $(p<0,001$, coef. $=2,17$, e.s. $=0,19, t=11,30)$.

Ainsi, les résultats confirment très largement la hiérarchie observée dans le cadre de la première question, avec une nette préférence pour le [E: allongé dans le contexte de la présentation des journaux télévisés, suivi de la variante légère- 
ment diphtonguée $\left[\mathrm{a}^{3}\right]$, le $[\varepsilon]$ semblant même être largement inadéquat, étant donné qu'aucun informateur ne le choisit.

\section{Question 3 : prononciation à enseigner dans les cours de français langue étrangère (FLE)}

La dernière question, qui touche pour sa part à la prononciation perçue comme la plus adaptée à l'enseignement dans les cours de FLE, montre, elle aussi, des tendances très similaires : 64,58\% (62/96) des informateurs préférent en effet le [ع:] allongé, 34,38\% (33/96) la variante légèrement diphtonguée [a ${ }^{3}$ ], alors qu'une seule informatrice $(1,04 \%, 1 / 96)$ choisit le $[\varepsilon]$ bref. En plus de confirmer la signification statistique de la différence entre ces taux $(p<0,001)$, le modèle de régression montre également, comme dans le cas de la première question (cf. supra), que la variable du sexe influe sur ces résultats de manière significative $(p<0,001)$, les hommes ayant plus souvent tendance à privilégier la variante diphtonguée $\left[\mathrm{a}^{3}\right]$ que les femmes, même si eux aussi choisissent tout de même majoritairement la variante allongée [ع:] (cf. figure 154).

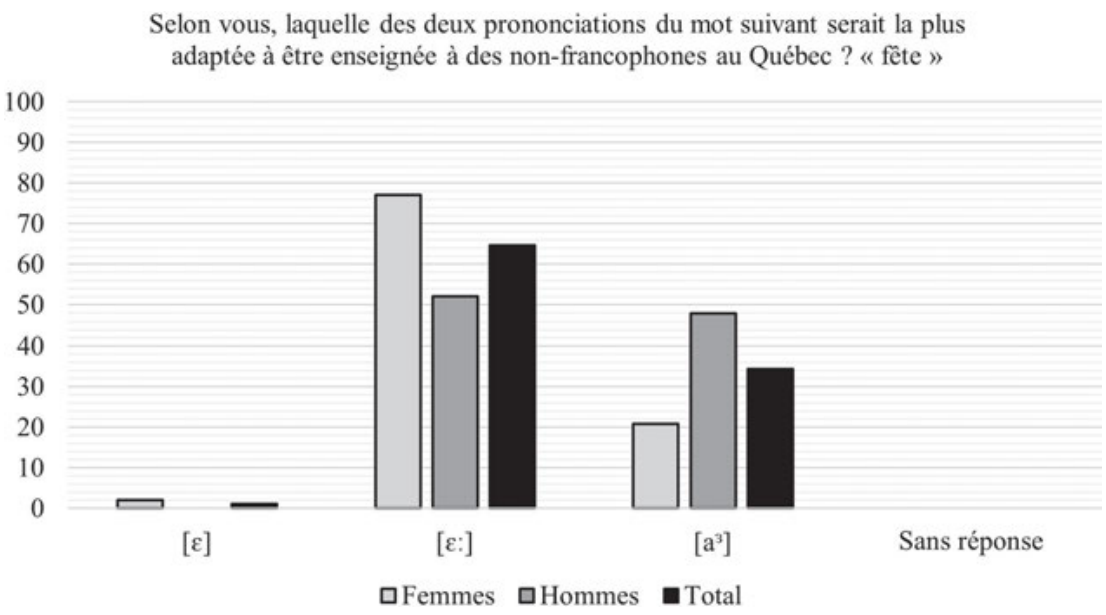

Figure 154 : Voyelle privilégiée (en \%) dans le mot <fête> pour les cours de français langue étrangère (FLE) $(n=96)$ - Mots isolés : réponses présentées dans leur globalité $(p<0,001$, coef. $=2,12$, e.s. $=0,23, t=9,08)$ ainsi que selon le sexe des informateurs $(p<0,001$, coef. $=-0,43$, e.s. $=0,09, t=4,72$ ).

Au-delà de la confirmation générale de la large préférence pour la variante [ع:] conjuguée à l'acceptation d'une légère diphtongaison et au rejet quasiment unanime de la variante brève $[\varepsilon]$, ces résultats confirment également la différence de comporte- 
ment évaluatif des femmes et des hommes observée dans le cadre de la première question (cf. supra) : les premières semblent largement préférer la variante allongée [ع:], alors que les seconds présentent une certaine hésitation entre cette même variante allongée [ $\left[\varepsilon^{\mathrm{r}}\right]$ et la variante diphtonguée $\left[\mathrm{a}^{3}\right]$. Or, étant donnée la tendance des hommes, bien connue en sociolinguistique, à préférer les traits régionaux présentant un certain prestige latent et la préférence des femmes pour les traits considérés comme standards présentant un prestige manifeste (cf. entre autres Labov 1963 ; Rovere 1982 ; Bourdieu 1982 ; cf. 2.1.2.2), il est vraisemblable que cette différence de sexe mette en lumière une différence de type de prestige de ces deux variantes : la variante allongée [ع:] semble représenter la variante standard au prestige manifeste, la variante diphtonguée $\left[\mathrm{a}^{3}\right]$ possédant apparemment un certain prestige latent.

En conclusion, les présents résultats ont permis d'affiner les hypothèses élaborées sur la base de l'analyse des productions : tout d'abord, l'opposition de durée $/ \varepsilon /: / \varepsilon$ : est clairement maintenue et stable dans la norme de prononciation québécoise. Par ailleurs, les différences entre les sexes découvertes dans ces évaluations perceptives semblent indiquer que la variante diphtonguée $\left[\mathrm{a}^{3}\right]$, « admise » au sein de cette norme lorsqu'elle est légère, présente un prestige non pas manifeste (comme la variante $/ \varepsilon: /$ ), mais latent.

\subsubsection{Ouverture et relâchement des voyelles /i, y, u/}

Le quatrième trait québécois ayant fait l'objet de tests de perception touche à l'ouverture et au relâchement des voyelles $/ \mathrm{i}, \mathrm{y}, \mathrm{u}$ / en finale des mots terminés par une ou plusieurs consonnes non allongeantes. ${ }^{1}$ L'analyse des productions a montré, à l'exemple du [I] relâché dans les mots six (liste de mots PFC) et villes (texte PFC), que ce trait est majoritairement maintenu dans l'usage des locuteurs-modèles. Ces résultats sont confrontés ci-dessous aux données de perceptions.

\section{Question 1 : évaluation (sans contexte explicite)}

De manière quelque peu surprenante, les résultats de la première question appliquée au mot isolé six montrent que les informateurs évaluent la variante tendue [i] et la variante relâchée [I] de manière très similaire : le [i] tendu atteint en effet un score moyen de 85,98\% (écart-type : 19,34\%), alors que celui du [I] est de 84,89\% (écart-type : 18,94\%), la petite différence entre les deux n'étant pas significative ( $p>0,05)$ (cf. figure 155).

1 Rappelons qu'il s'agit des consonnes suivantes : /p, b, t, d, k, g, f, s, J, m, n, n, l/ (cf. p. ex. Silviet-Carricart 2007, 17). 
Évaluation de [i] vs [1] dans six (liste de mots PFC)

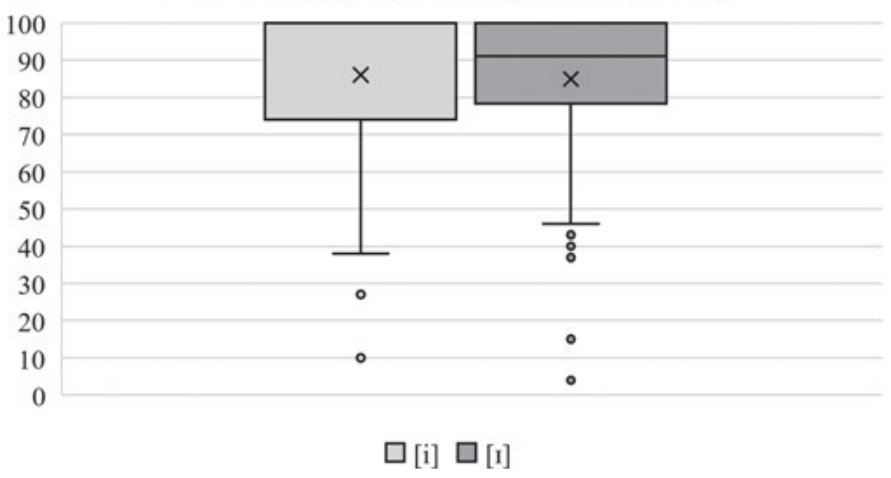

Figure 155 : Évaluation perceptive (en \%) des voyelles [i] et [1] par les informateurs québécois $(\mathrm{n}=96)$ dans le mot isolé $\langle$ six $>$ (ANOVA : $p>0,05)$.

Notons que l'analyse de variance indique un effet significatif du niveau d'éducation sur les résultats du stimulus comportant un [i] tendu $(F=4,56, p<0,05)$, les informateurs dont le niveau d'éducation est plus élevé (diplôme universitaire ou DEC des programmes d'études pré-universitaires) ayant tendance à évaluer cette variante plus positivement (moyenne : 89,66\% ; écart-type : 18,63\%) que les titulaires d'un diplôme de niveau moins élevé (primaire, secondaire, école de métiers ou DEC des programmes d'études techniques) (moyenne : 81,52\% ; écart-type : 19,41\%).

Les résultats touchant au syntagme autour des mêmes villes tiré du texte PFC présentent des résultats très similaires, le [i] tendu obtenant un score moyen de 84,37\% (écart-type : 17,94\%), contre 89,13\% (écart-type : 14,39\%) pour le [I] relâché, la différence entre ces taux n'étant, à nouveau, pas significative $(p>0,05)$ (cf. figure 156).

L'analyse de variance indique à nouveau un effet significatif, mais il porte dans le cas présent sur le facteur du sexe, qui influence les résultats du stimulus tendu [i] $(F=5,86, p<0,05)$ : les informatrices ont en effet tendance à évaluer cette variante légèrement mieux (moyenne : 87,69\% ; écart-type 16,44\%) que les informateurs masculins (moyenne : 80,07\% ; écart-type : 19,02\%).

Deux observations peuvent être tirées des résultats de cette première question : premièrement, étant donné que la question a été posée séparément pour chaque stimulus, c'est-à-dire sans comparaison directe des deux variantes, il est probable que les résultats très similaires des deux stimuli montrent que la différence entre ces variantes est simplement peu saillante dans la perception des informateurs québécois. Ceci expliquerait en effet pourquoi les informateurs évaluent intuitivement, dans les deux cas, les deux stimuli de manière très similaire. Deuxièmement, les évaluations plus positives des variantes tendues [i] par les 


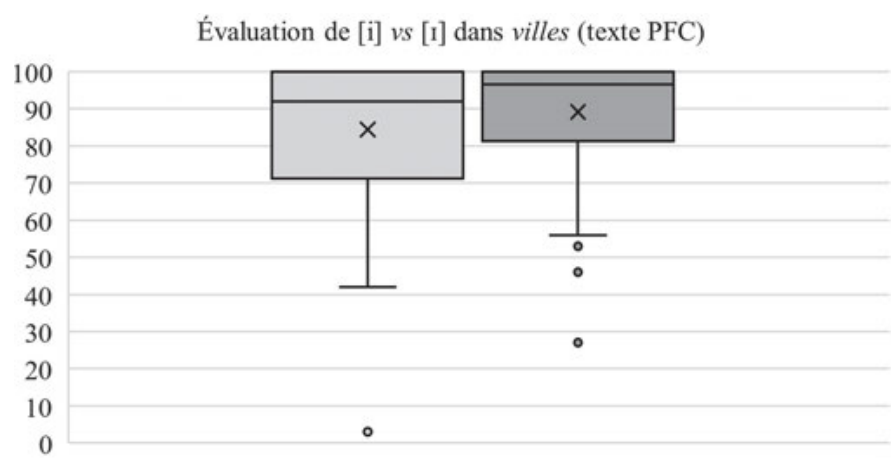

$[$ [i] $\square[1]$

Figure 156 : Évaluation perceptive (en \%) des voyelles [i] et [1] par les informateurs québécois $(\mathrm{n}=96)$ dans le mot <villes> tiré du texte PFC $($ ANOVA $: p>0,05)$.

femmes et les informateurs au niveau d'éducation plus élevé semblent, ici aussi, être attribuables à une certaine insécurité linguistique. Ces deux groupes sociodémographiques sont en effet connus en sociolinguistique pour être particulièrement affectés par cette insécurité (cf. 4.2.2) et ce résultat converge également avec l'insécurité linguistique observée dans les questionnaires touchant aux représentations et attitudes de ces mêmes sous-groupes (cf. 4.3.2), ainsi que dans les perceptions de l'opposition /a/ : /a:/ (cf. 6.3.1).

\section{Question 2 : prononciation idéale des présentateurs de journaux télévisés}

Les résultats de la deuxième question, dans laquelle les deux stimuli sont comparés directement, présentent des tendances plus claires. La variante relâchée [I] est en effet préférée par une large majorité des informateurs : 58,33\% (56/96) d'entre eux la choisissent, contre seulement 30,21\% (29/96) qui préfèrent la variante tendue [i], cette différence étant hautement significative $(p<0,001)$. Par ailleurs, ce résultat semble relativement homogène parmi les différents groupes sociodémographiques, le modèle de régression ne montrant aucun effet significatif $(p>0,05)$ des quatre variables sociodémographiques prises en compte (sexe, âge, origine, niveau d'éducation) (cf. figure 157).

Les résultats obtenus par le syntagme autour des mêmes villes sont très similaires, avec une variante relâchée [I] préférée par 62,50\% (60/96) des informateurs, contre $32,29 \%$ (31/96) pour la variante tendue [i]. Le modèle de régression indique, ici aussi, que la différence entre ces deux taux est hautement significative $(p<0,001)$ alors que d'autre part, les variables sociodémographiques n'influent pas significativement sur ces résultats $(p>0,05)$ (cf. figure 158). 
Selon vous, comment un présentateur de télévision québécois devrait-il prononcer le mot suivant? « six »

100
90
80
70
60
50
40
30
20
10
0

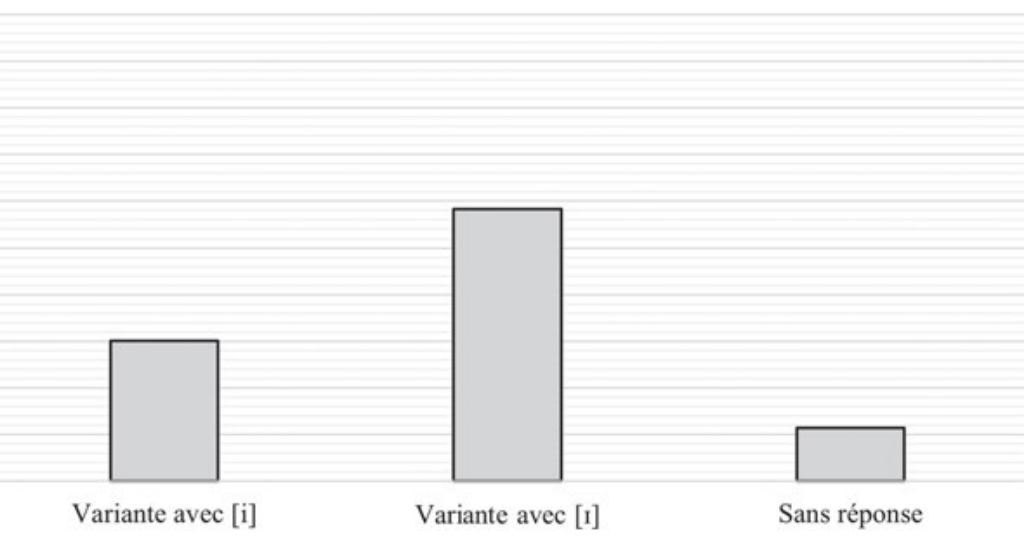

Figure 157 : Voyelle privilégiée (en \%) dans le mot <six> pour les présentations de journaux télévisés $(\mathrm{n}=96)$ - Mots isolés ( $p<0,001$, coef. $=1,60$, e.s. $=0,29, t=5,49$ ).

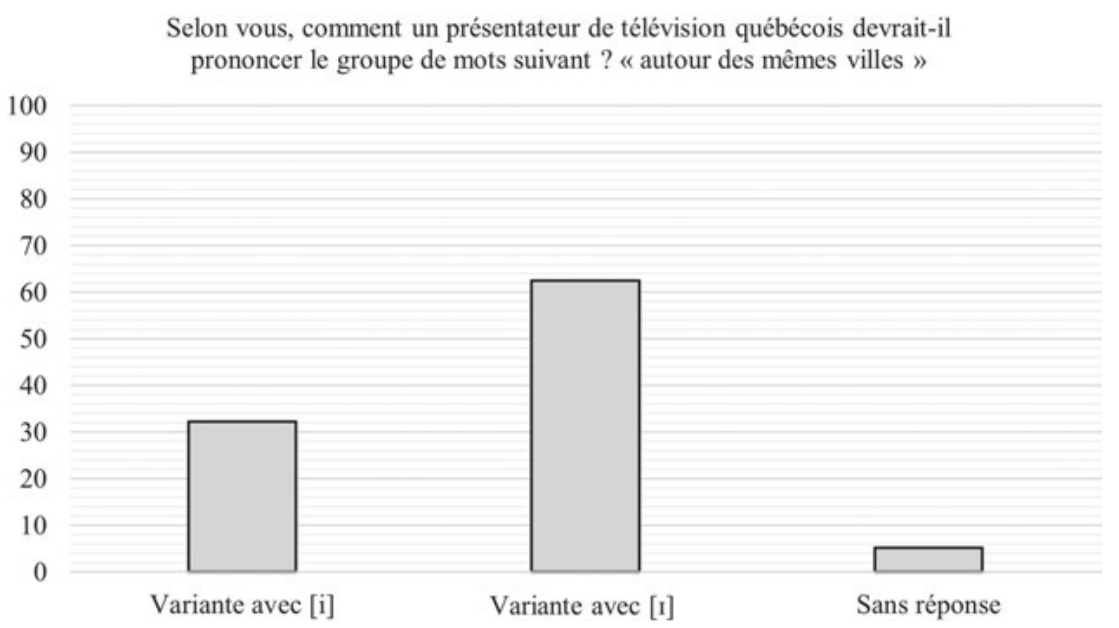

Figure 158 : Voyelle privilégiée (en \%) dans le mot <villes> pour les présentations de journaux télévisés $(\mathrm{n}=96)$ - Texte PFC $(p<0,001$, coef. $=1,26$, e.s. $=0,25, t=5,00)$.

Ainsi, contrairement à ceux de la première question, qui ne montraient pas de réelle préférence des informateurs pour l'une ou l'autre variante (cf. supra), les résultats de cette deuxième question sont plutôt clairs : les informateurs préfèrent très largement la variante relâchée [I]. Cette différence est vraisemblablement explicable par la conception des questions, la seconde comparant 
directement les deux variantes dans le cadre d'une question unique alors que les stimuli étaient évalués séparément dans le cadre de la première. Ainsi, dans le cas présent, dans lequel la saillance du trait est moins important car les stimuli peuvent être écoutés directement l'un après l'autre, les informateurs semblent largement privilégier la variante endogène québécoise.

\section{Question 3 : prononciation à enseigner dans les cours de français langue étrangère (FLE)}

Les résultats de la dernière question, qui touche à la prononciation-cible pour le FLE, montrent, pour leur part, des tendances très similaires à ceux de la deuxième question, avec dans l'ensemble une préférence pour la variante relâchée [I], choisie par 51,04\% (49/96) des informateurs, contre 37,50\% (36/96) pour le [i] tendu. Le modèle de régression montre que cette différence de taux est significative à $p<0,001$ et révèle d'autre part un effet significatif du sexe $(p<0,01)$, les femmes ayant davantage tendance à préférer la variante tendue [i] $(43,75 \%, 21 / 48)$ que les hommes $(31,25 \%, 15 / 48)$ (cf. figure 159).

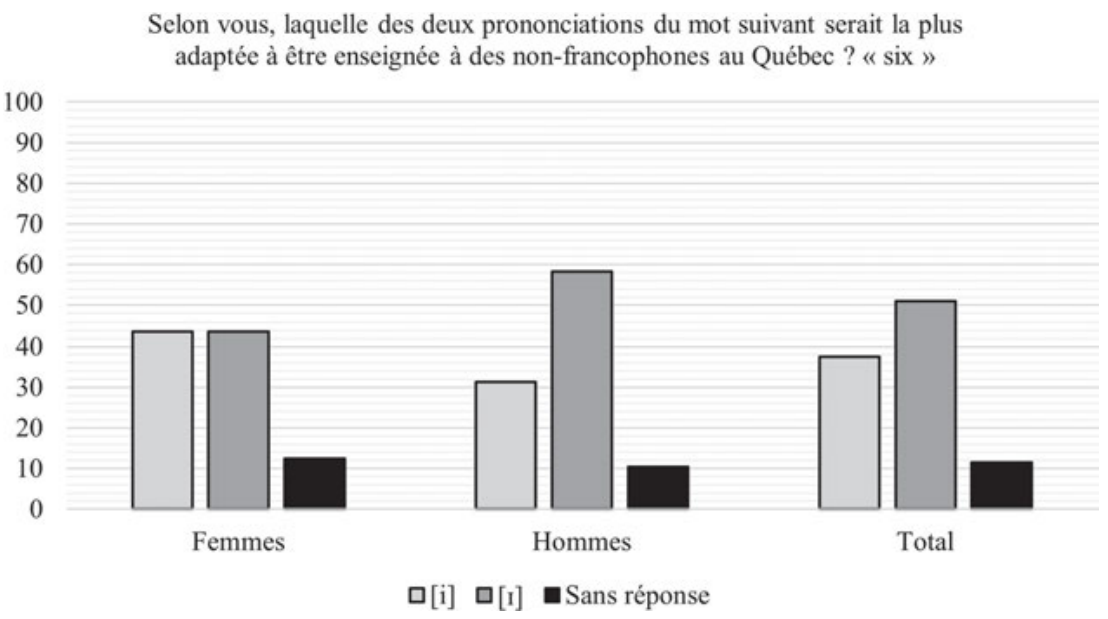

Figure 159 : Voyelle privilégiée (en \%) dans le mot <six> pour les cours de français langue étrangère $(\mathrm{FLE})(\mathrm{n}=96)$ - Mots isolés ; réponses présentées dans leur globalité $(p<0,001$, coef. $=3,16$, e.s. $=0,29, t=10,88$ ) ainsi que selon le sexe des informateurs ( $p<0,01$, coef. $=-0,32$, e.s. $=0,11$, $t=-2,83)$.

La différence liée au sexe observée dans le cadre de la première question (cf. supra) semble donc se confirmer ici, les femmes hésitant plus entre les deux variantes. Cette hésitation pourrait être à nouveau attribuable à une certaine insé- 
curité linguistique. Ceci est d'autant plus probable que dans cette question, les deux variantes sont comparées directement, de sorte que les perceptions des informateurs pourraient avoir été influencées par les représentations géographiques (cf. 3.1 ; cf. également Lippi-Green 2012 ; Moyer 2013 ; Didelot 2019) attribuées par les auditeurs québécois à la variante [i], qu'ils associent à un français " parisien » ou « européen » (de manière similaire à ce qui a pu être observé dans le cas de la Suisse romande, cf. 6.2.6). Ce résultat secondaire ne remet cependant pas en question le résultat global, la variante endogène relâchée [I] étant ici aussi préférée à la variante exogène tendue [i].

Les résultats obtenus par le syntagme autour des mêmes villes renforcent cette tendance : la variante avec [I] relâché est préférée par 73,96\% (71/96) des informateurs, alors que seuls 22,92\% (22/96) d'entre eux optent pour la variante avec [i] tendu, cette différence de taux étant hautement significative $(p<0,001)$ (cf. figure 160).

Selon vous, laquelle des deux prononciations du groupe de mots suivant serait la plus adaptée à être enseignée à des non-francophones au Québec ? « autour des mêmes villes »

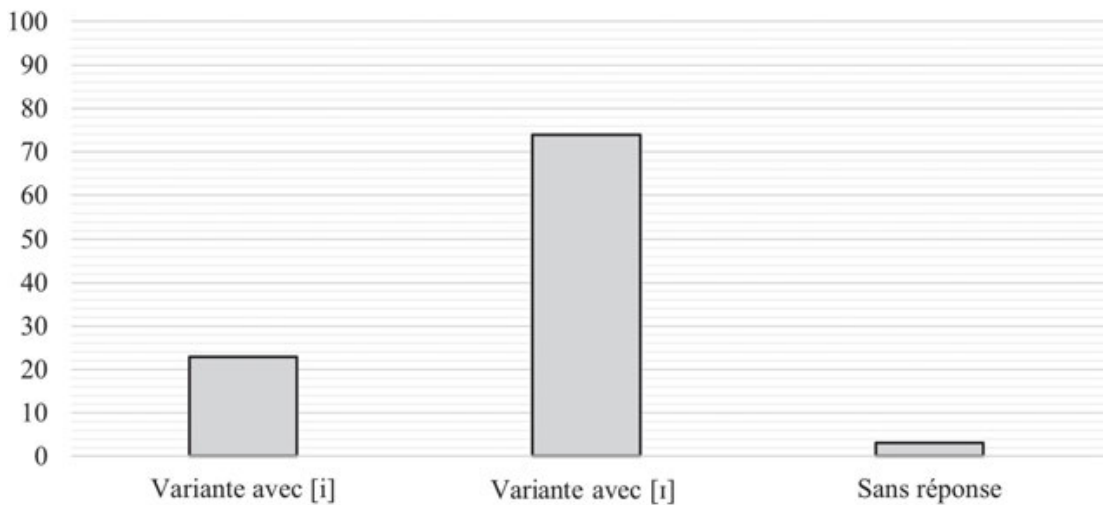

Figure 160 : Voyelle privilégiée (en \%) dans le mot <villes> pour les cours de français langue étrangère (FLE) $(\mathrm{n}=96)$ - Texte PFC ( $p<0,001$, coef. $=1,21$, e.s. $=0,24, t=4,95)$.

En conclusion, trois observations peuvent être tirées de ces résultats : premièrement, dans une situation de comparaison directe des variantes [I] et [i] en finale de mot terminé par une consonne non allongeante, les Québécois semblent clairement préférer la variante relâchée [I], ce qui confirme les résultats touchant aux productions des locuteurs-modèles. Aussi semble-t-il possible de considérer que le relâchement du /i/ fait partie de la norme de prononciation québécoise. Deuxièmement, les résultats liés aux variables sociodémographiques ont cependant 
montré également que le [i] tendu, associé au français parisien dans ce contexte, engendre encore une certaine insécurité linguistique parmi les informateurs, en particulier chez les femmes. Par ailleurs, le taux d'informateurs préférant cette variante tendue [i] étant relativement élevé, en particulier dans les questions 2 et 3 (cf. supra), il semble tout de même exister encore un certain prestige manifeste de cette variante dans la norme de prononciation québécoise, parallèlement au prestige manifeste tout à fait établi et stable du [I] relâché. Troisièmement, la différence entre les variantes [I] et [i] ne semble pas particulièrement saillante, étant données les évaluations très similaires des deux mêmes stimuli lorsqu'ils ne sont pas comparés directement au sein d'une seule et même question.

\subsubsection{Dévoisement (ou effacement) des voyelles /i, y, u/}

Le cinquième trait ayant fait l'objet des tests de perception au Québec concerne le dévoisement et l'effacement des mêmes voyelles /i/, / y/ et / $\mathrm{u} /$ entre deux consonnes sourdes. L'analyse des productions a montré, d'une part, une variation considérable entre les variantes voisées, dévoisées et syncopées chez les locuteurs-modèles et, d'autre part, une légère tendance majoritaire au voisement $\mathrm{du} / \mathrm{i} / \mathrm{dans}$ ce contexte. $\mathrm{Au}$ contraire des autres traits endogènes analysés, ces résultats incitent donc a priori à ne pas retenir ce trait dans la définition de la norme de prononciation québécoise, des résultats qui devront être confirmés dans le cadre des tests de perception. Ces tests se basent sur la variation observée dans le mot vérifications (/i/ voisé : [vebifikasjõ] vs. /i/ syncopé : [vebif:kasjũ]), testé dans le syntagme des vérifications d'identité tiré du texte PFC. Notons qu'aucun mot isolé tiré des listes n’a pu être pris en compte pour ces tests. En effet, lors des analyses de production la quasi-totalité de ces mots (piquet, piqué, piquais, piquer) ont été réalisés - probablement en raison d'une articulation exagérée et d'un débit ralenti dus à l'audio-monitoring (cf. Labov 1972) - avec un /i/ voisé (cf. 5.3.1.6), ce qui a rendu impossible la comparaison entre variantes avec /i/ voisé et /i/ dévoisé ou syncopé.

\section{Question 1 : évaluation (sans contexte explicite)}

Les résultats de la première question, tout d'abord, confirment globalement ceux de l'analyse des productions : le maintien d'un /i/ voisé, déjà majoritaire dans l'usage des locuteurs-modèles, est préféré dans les évaluations perceptives des informateurs. La variante avec /i/ voisé obtient en effet un score de 88,76\% (écarttype : $17,35 \%$ ), alors que la syncope de ce /i/ obtient un score légèrement plus bas de $84,20 \%$ (écart-type : 16,97\%), la différence entre ces scores étant légèrement significative à $p<0,05$ selon l'analyse de variance (cf. figure 161). 
Évaluation $\mathrm{du} / \mathrm{i} /$ voisé et du /i/ syncopé dans vérifications (texte PFC)

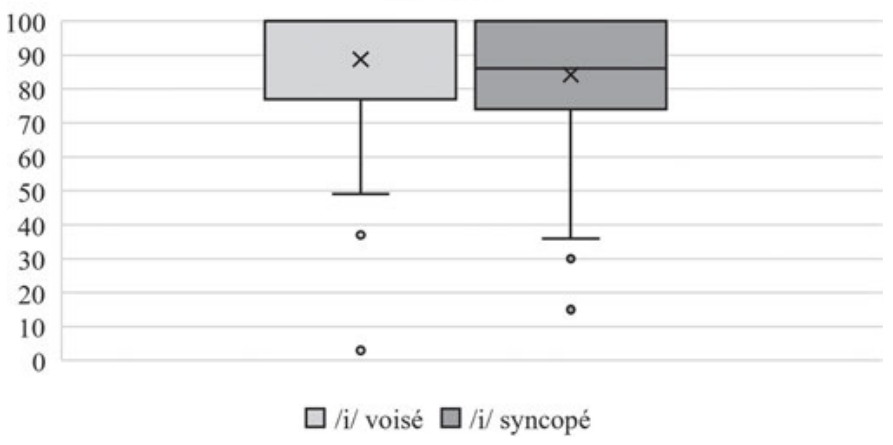

Figure 161 : Évaluation perceptive (en \%) du /i/ voisé et syncopé dans le mot <vérifications> tiré du texte PFC $(\mathrm{n}=96)($ ANOVA $: p<0,05)$.

Ainsi, contrairement à tous les autres traits testés jusqu'ici dans le cas du Québec, les évaluations perceptives des informateurs québécois présentent bien une tendance n'allant pas en direction de la variante endogène (/i/ syncopé), mais de la variante de référence (/i/ voisé). Ce résultat va ainsi dans le sens de ceux de Cox (1998) et à l'encontre des observations de toutes les autres études préalables (cf. Dumas 1987 ; Reinke 2004 ; 2005 ; Ostiguy/Tousignant 2008), qui considéraient que la syncope d'un tel /i/ passe inaperçue dans la perception des Québécois. Ce n'est visiblement pas le cas ici, la syncope du /i/ étant significativement moins bien évaluée que son maintien. Ceci est d'autant plus pertinent que dans cette première question, l'évaluation a été faite dans le cadre d'une question séparée pour chaque stimulus (au contraire des questions 2 et 3).

\section{Question 2 : prononciation idéale des présentateurs de journaux télévisés}

Les résultats de la question abordant la prononciation-cible pour les présentateurs de journaux télévisés montrent des tendances très similaires : une majorité de 53,13\% (51/96) des informateurs préfère en effet la variante avec /i/ voisé, alors que seuls 32,29\% (31/96) d'entre eux portent leur choix sur la variante endogène avec /i/ syncopé, cette différence étant hautement significative $(p<0,001)$ (cf. figure 162).

La répartition des réponses parmi les différents groupes sociodémographiques semble, pour sa part, relativement équilibrée, le modèle de régression n'indiquant aucun effet significatif des quatre facteurs sociodémographiques pris en compte $(p>0,05)$. Globalement, ces résultats confirment ainsi les observations faites dans le cadre la première question : contrairement aux autres traits testés 
Selon vous, comment un présentateur de télévision québécois devrait-il prononcer le groupe de mots suivant ? «des vérifications d'identité »

100

90

80

70

60

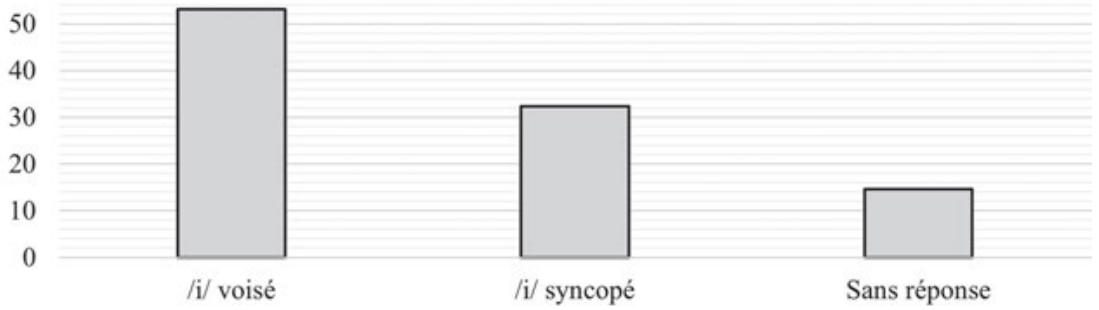

Figure 162 : Variante privilégiée (en \%) dans le mot <vérifications> pour les présentations de journaux télévisés $(\mathrm{n}=96)$ - Texte PFC $(p<0,001$; coef. $=2,13$; e.s. $=0,32 ; t=6,68)$.

pour le Québec, le relâchement du /i/ entre deux consonnes sourdes semble être perçu par une majorité des informateurs comme non standard.

\section{Question 3 : prononciation à enseigner dans les cours de français langue étrangère (FLE)}

Les tendances au maintien du /i/ voisé indiquées par les résultats des deux premières questions sont également confirmées dans la troisième, qui touche, rappelons-le, à la prononciation-cible en cours de FLE : 52,08\% (50/96) des informateurs choisissent en effet cette variante ([vesifikasjj̃]), alors que seuls 35,42\% (34/96) d'entre eux optent pour celle dans laquelle le /i/ est syncopé ([vesif:kasjõ]). Par ailleurs, dans le cas présent, le modèle de régression indique également que les femmes et les hommes présentent des réponses significativement différentes $(p<0,001)$ : les femmes choisissent majoritairement - dans le sens de la majorité des informateurs - la variante dans laquelle le /i/ voisé est maintenu (/i/ voisé : 58,33\%, 28/48; /i/ syncopé : 20,83\%,10/48), alors que les réponses des hommes présentent plus d'ambiguïté entre les deux catégories (/i/ voisé : 45,83\%, 22/48 ; /i/ syncopé : 50,00\%, 24/48) (cf. figure 163).

Notons que la différence observée entre les réponses des femmes et des hommes pourrait être (de manière similaire aux variantes $/ \varepsilon:$ et $/ \mathrm{a}^{3} /$ dans fête ; cf. 6.3.3) un indice d'une différence de prestige, avec un /i/ voisé présentant un prestige manifeste (variante largement préférée par les femmes) et un /i/ syncopé détenant un prestige latent (variante légèrement préférée par les hommes). Cette 
Selon vous, laquelle des deux prononciations du groupe de mots suivant serait la plus adaptée à être enseignée à des non-francophones au Québec ? "des vérifications d'identité »

100
90
80
70
60
50
40
30
20
10
0

(1)

(1)

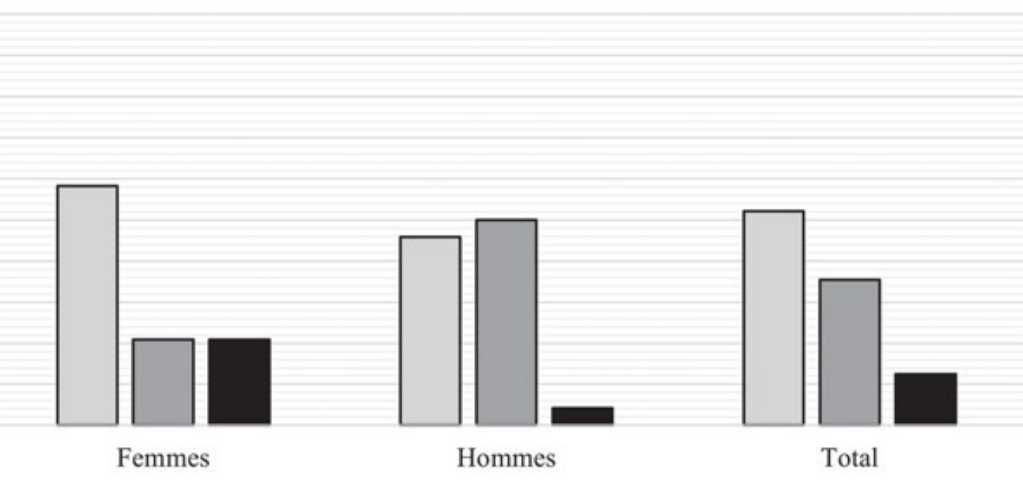

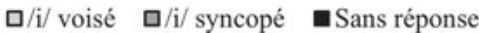

Figure 163 : Variante privilégiée (en \%) dans le mot <vérifications> pour les cours de français langue étrangère (FLE) $(\mathrm{n}=96)$ - Texte PFC; réponses présentées dans leur globalité $(p<0,001$, coef. $=2,72$, e.s. $=0,29, t=9,52)$ ainsi que selon le sexe des informateurs $(p<0,001$, coef. $=-0,46$, e.s. $=0,11, t=-4,13)$.

différence de prestige est d'autant plus probable que, contrairement au cas du relâchement de la voyelle /i/ (cf. 6.3.4), il ne peut pas s'agir d'un problème de non-saillance du trait, étant donné que la première question, dans laquelle les stimuli ne sont pas comparés directement, montre bien un écart significatif dans les évaluations de ces stimuli, la différence étant donc bel et bien perçue par les informateurs.

En conclusion, les résultats obtenus pour ce trait peuvent être résumés comme suit : tout d'abord, la syncope du /i/ entre deux consonnes sourdes n'est pas préférée par les informateurs québécois. Étant donné que l'analyse des productions a montré que le /i/ voisé est également majoritairement maintenu dans l'usage des locuteurs-modèles, le présent trait ne peut être considéré comme faisant partie de la norme de prononciation québécoise. Par ailleurs, dans la même ligne argumentative, les différences en fonction du sexe révélées à propos de la troisième question suggèrent que les deux variantes testées pourraient présenter deux types de prestige différents : prestige manifeste pour le /i/ voisé, latent pour le /i/ syncopé. Ce dernier résultat, qui reste hypothétique, devrait encore être vérifié à l'avenir dans le cadre d'expériences de perception supplémentaires, dans la mesure où la tendance n'a pu être observée que dans l'une des trois questions. 


\subsubsection{Opposition / $\tilde{\mathrm{e}} /: / \tilde{\varepsilon} /$}

Le premier trait touchant à des voyelles nasales testé perceptivement au Québec concerne le maintien de l'opposition /œ̃̃/ : / $\tilde{\varepsilon} /$. À ce propos, l'analyse des productions a montré, à l'exemple des mots brun et un, que la variante arrondie [õ] est utilisée quasiment unanimement par les locuteurs-modèles en correspondance avec la graphie <un> (cf. 5.3.2.1), confirmant ainsi l'accord déjà unanime des études précédentes par rapport au maintien de la voyelle nasale /œẽ/ (cf. 2.3.4). Les tests de perception, sur la base desquels ces résultats seront vérifiés, examinent le maintien de l'opposition sur la base des deux mêmes mots, brun en tant que mot isolé (liste de mots PFC) et un dans le syntagme d'un autre côté (texte PFC).

\section{Question 1 : évaluation (sans contexte explicite)}

Les résultats de la première question portant sur le mot isolé brun confirment très largement les résultats des analyses des productions des locuteurs-modèles : malgré une comparaison indirecte des deux stimuli, c'est-à-dire dans le cadre de deux évaluations perceptives séparées, la variante arrondie [œẽ] est très largement préférée : elle obtient en effet un score de 94,54\% (écart-type : 10,63\%), alors que la variante non arrondie, visiblement considérée comme non acceptable par une grande majorité des informateurs, obtient un score très faible de $29,90 \%$ (écarttype : $21,64 \%$ ). Sans surprise, étant donnée la clarté de ces résultats, l'analyse de variance indique que la différence entre ces scores est hautement significative $(p<0,001)$. Soulignons par ailleurs la différence considérable entre les écart-types

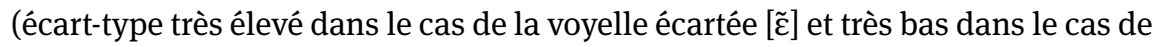
la voyelle arrondie [õ]]), qui représente un indice supplémentaire d'une certaine insécurité des informateurs dans l'évaluation du [ $\tilde{\varepsilon}]$ et, au contraire, une grande sécurité dans l'évaluation de [õ̃] (cf. figure 164).

Les résultats touchant au syntagme d'un autre côté ne confirment cependant

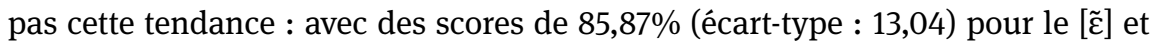
de $88,31 \%$ (écart-type : 16,30\%) pour le [õ]], la différence entre les évaluations est trop faible pour s'avérer significative $(p>0,05)$. Il en va de même pour les quatre facteurs pris en compte dans l'analyse de variance, qui ne présentent pas d'effet significatif sur ces résultats ( $p>0,05$ ) (cf. figure 165).

La différence entre les résultats relevés pour le mot isolé brun et le mot un dans le syntagme d'un autre côté pourrait être expliquée par la combinaison de deux facteurs : premièrement, les différents mots utilisés pourraient jouer un rôle considérable dans cette évaluation : en effet, l'analyse des productions des locuteurs-modèles a permis de montrer que globalement, seul le déterminant

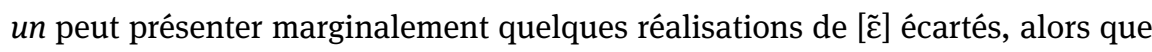




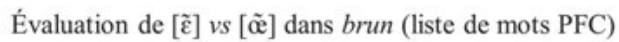

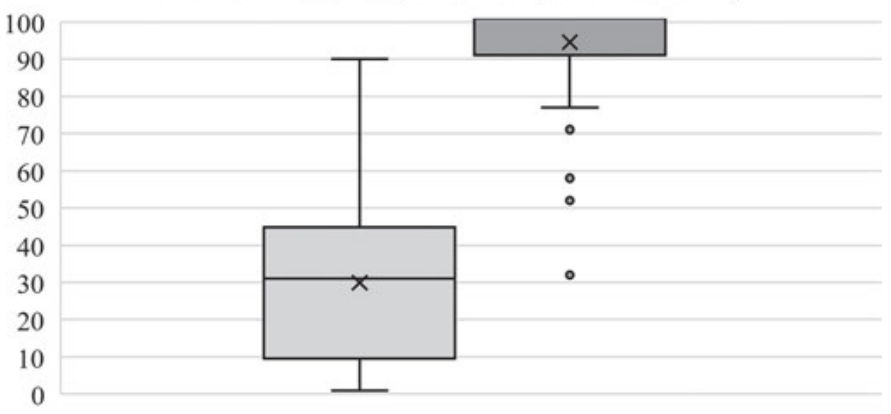

$[\tilde{\varepsilon}] \square[\tilde{\tilde{\varepsilon}}]$

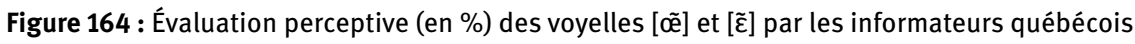
$(\mathrm{n}=96)$ dans le mot isolé $<$ brun $>($ ANOVA $: p<0,001)$.

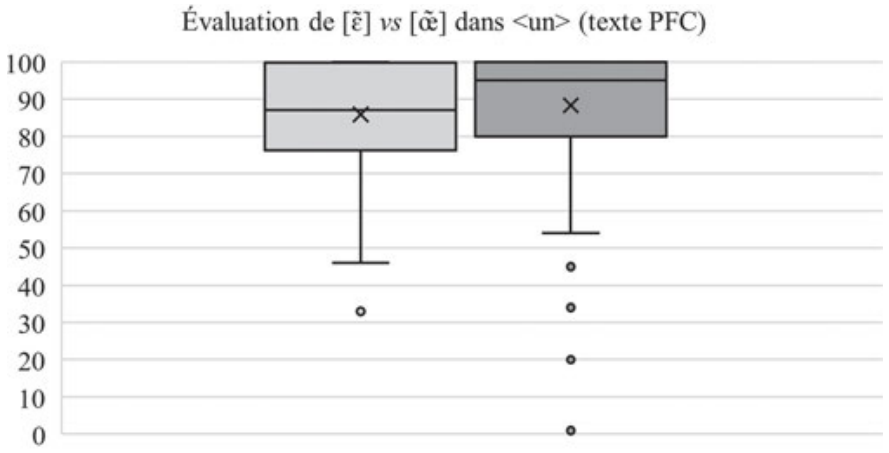

\section{$[\tilde{\varepsilon}] \square[\tilde{\propto}]$}

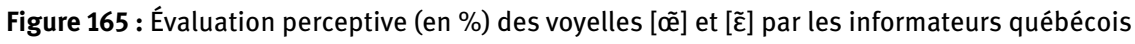
$(\mathrm{n}=96)$ dans le mot $<$ un> tiré du texte PFC (ANOVA $: p>0,05)$.

brun est quasiment unanimement réalisé avec un [õ] arrondi. Il est donc vraisem-

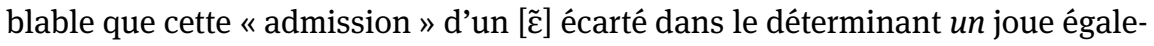
ment un rôle dans les évaluations perceptives. Deuxièmement, il est très probable qu'à un niveau méthodologique, comme à plusieurs reprises dans les trois tests de perception, le stimulus syntagmatique utilisé (d'un autre côté) ait perturbé les informateurs dans leurs évaluations.

Ainsi, compte tenu de ces problèmes méthodologiques dans le cas du stimulus syntagmatique, le résultat global très clair touchant au mot isolé brun ne semble pas remis en question, l'opposition /œẽ/: / $\tilde{\varepsilon} /$ paraissant être largement maintenue dans la perception des informateurs québécois. Gardons cependant 
en mémoire que ces évaluations perceptives pourraient également indiquer qu'il existe bel et bien une différence lexicale dans le maintien de cette opposition, le déterminant admettant de manière très marginale certains [ [̃] écartés.

\section{Question 2 : prononciation idéale des présentateurs de journaux télévisés}

Les résultats de la question sur la prononciation idéale d'un présentateur de télévision renforcent largement la conclusion globale de la première question : ils ne pourraient en effet être plus clairs, puisque les informateurs préfèrent la voyelle nasale arrondie [õ̃] à l'unanimité (100\%, 96/96). Étant donné le choix de cette unique catégorie par les informateurs, il va de soi qu'il n'a été ni possible ni nécessaire d'appliquer un test de signification aux données (cf. figure 166).

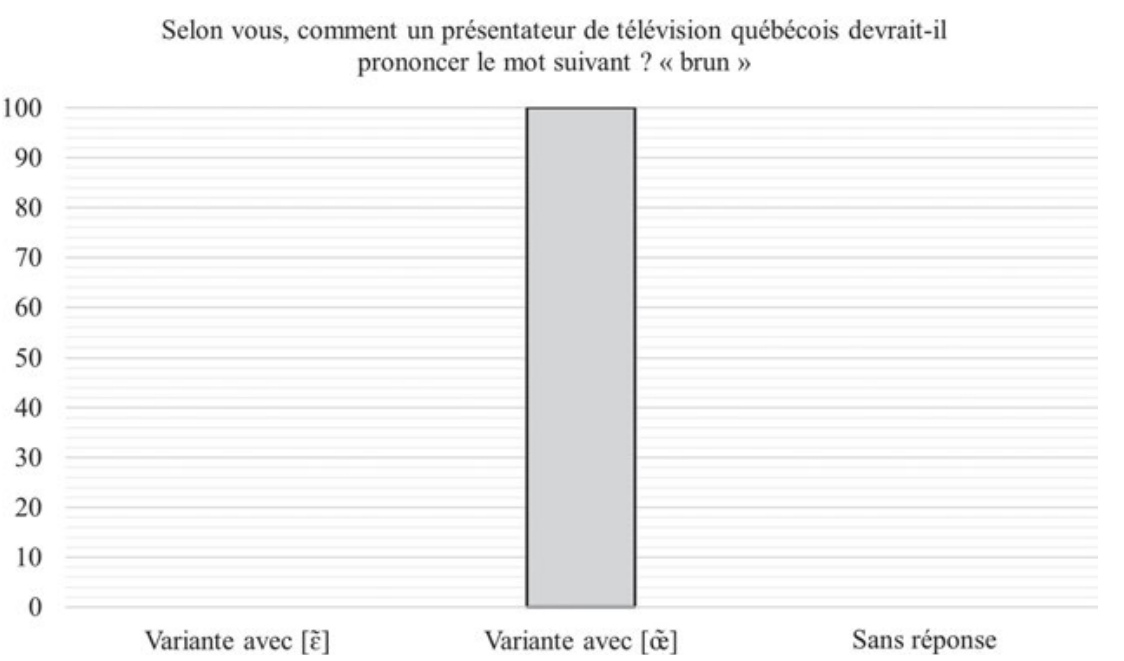

Figure 166 : Voyelle privilégiée (en \%) dans le mot <brun> pour les présentations de journaux télévisés $(\mathrm{n}=96)$ - Mots isolés.

Pour le syntagme d'un autre côté, les résultats révèlent également une tendance allant clairement en direction de la variante arrondie [œẽ], même si, comme on pouvait s'y attendre, celle-ci n'est pas aussi univoque que dans le cas du mot isolé brun : 68,75\% (66/96) des informateurs préfèrent en effet le [õ̃] arrondi, alors

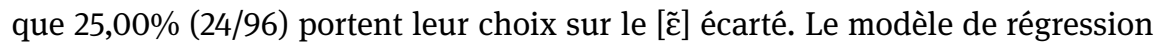
montre, d'une part, que cette différence est hautement significative $(p<0,001)$ et, d'autre part, que les variables sociodémographiques ne présentent aucun effet significatif sur les résultats ( $p>0,05)$ (cf. figure 167). 
Selon vous, comment un présentateur de télévision québécois devrait-il prononcer le groupe de mots suivant ?

« D'un autre côté »

100

90

80

70

60

50

40

30

20

10

0

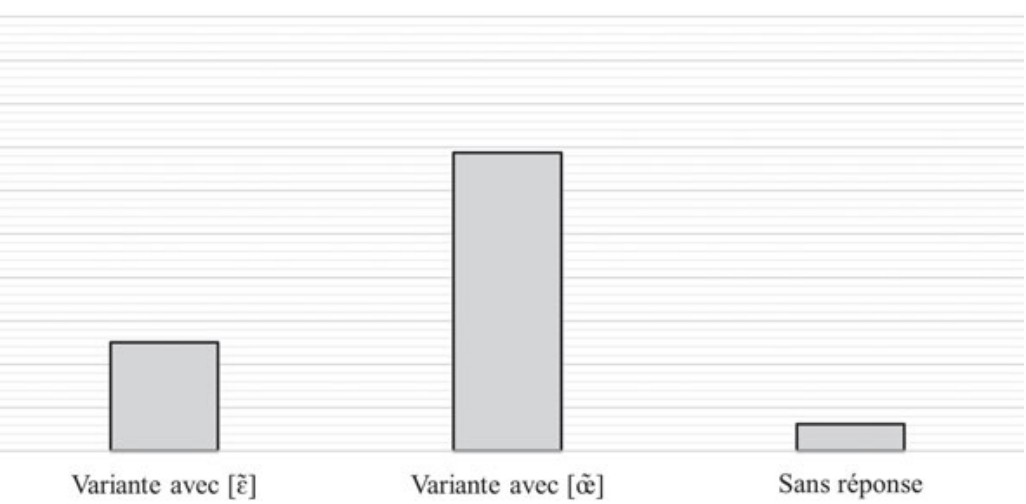

Figure 167 : Voyelle privilégiée (en \%) dans le mot <un> pour les présentations de journaux télévisés $(\mathrm{n}=96)$ - Texte PFC ( $p<0,001$, coef. $=1,27$, e.s. $=0,26, t=4,80)$.

Les constats faits par rapport à cette question sont donc les mêmes que pour la première question : globalement, l'opposition /oẽ/ : / $\tilde{\varepsilon} /$ semble se maintenir dans la perception des informateurs, et ce, aussi bien dans le mot brun que dans le mot $u n$. La différence d'évaluation entre le mot isolé brun et le syntagme incluant un peut être expliquée par la longueur du stimulus syntagmatique (= problème méthodologique) et par la plus grande résistance de brun au changement (= différence lexicale). Par ailleurs, la différence entre les résultats obtenus pour le stimulus syntagmatique dans le cadre de la première question (pas de tendance significative) et de la présente (forte tendance en faveur du [õ] arrondi) peut être attribuée au fait que dans le cas présent, les stimuli sont comparés directement au sein d'une seule et même question, ce qui facilite l'évaluation pour les informateurs.

\section{Question 3 : prononciation à enseigner dans les cours de français langue étrangère (FLE)}

Les tendances sont également très similaires dans la question portant sur le FLE : les réponses des informateurs touchant au mot isolé brun se portent également unanimement sur la variante arrondie [oẽ] (100\%, 96/96), raison pour laquelle aucun test de signification n'est nécessaire (cf. figure 168).

Le syntagme engendre, quant à lui, à nouveau les mêmes tendances, la variante [œẽ] étant largement préférée : 66,67\% (64/96) des informateurs choisissent cette variante, $28,13 \%$ la variante écartée [ $\tilde{\varepsilon}]$. Le modèle de régression indique que la différence entre ces deux taux est significative à $p<0,001$ (cf. figure 169). 
Selon vous, laquelle des deux prononciations du mot suivant serait la plus adaptée à être enseignée à des non-francophones au Québec ? « brun »

100
90
80
70
60
50
40
30
20
10
0

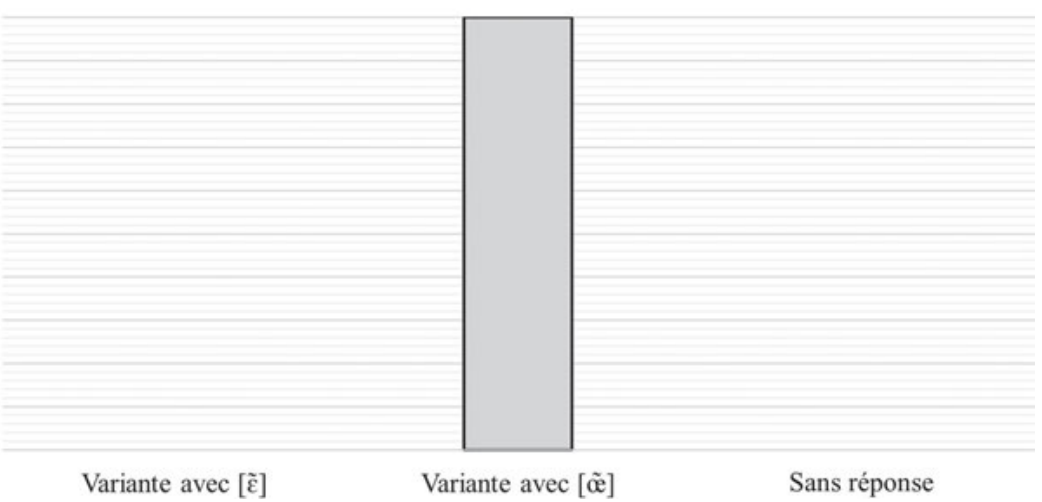

Figure 168 : Voyelle privilégiée (en \%) dans le mot <brun> pour les cours de français langue étrangère (FLE) $(n=96)$ - Mots isolés.

Selon vous, laquelle des deux prononciations du groupe de mots suivant serait la plus adaptée à être enseignée à des non-francophones au

Québec ? « autour des mêmes villes »

$$
\begin{array}{r}
100 \\
90 \\
80 \\
70 \\
60 \\
50 \\
40 \\
30 \\
20 \\
10
\end{array}
$$

0

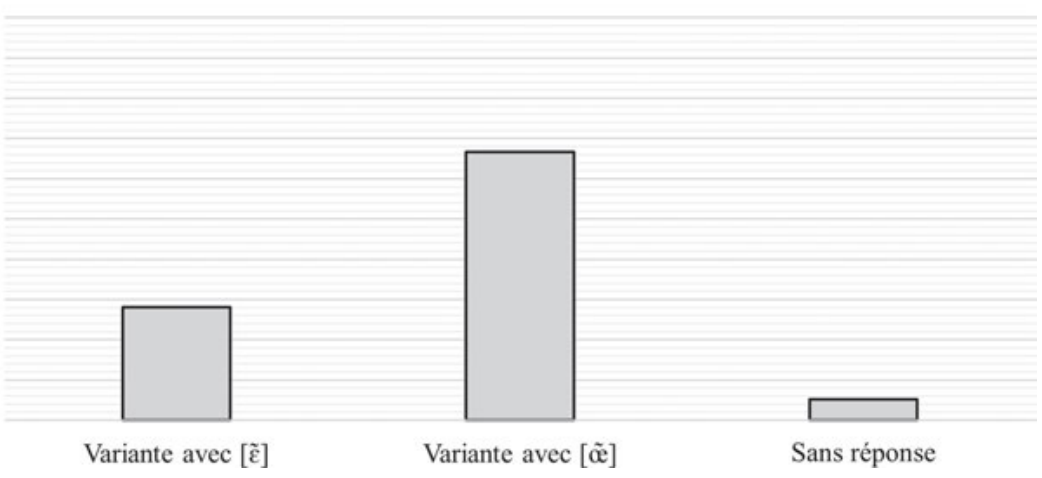

Figure 169 : Voyelle privilégiée (en \%) dans le mot <brun> pour les cours de français langue étrangère (FLE) $(\mathrm{n}=96)$ - Texte PFC ( $p<0,001$, coef. $=2,00$, e.s. $=0,30, t=6,77)$.

En conclusion, deux observations peuvent donc être tirées des tests de perception effectués par rapport à l'opposition /oẽ/ : / $\tilde{\varepsilon} /:$ tout d'abord, parallèlement à ce qu'ont montré les analyses des productions des locuteurs-modèles, l'opposition est bien maintenue dans la perception des informateurs québécois, et ce, tant dans le mot brun que dans le mot un. Elle peut donc être considérée comme partie intégrante et stable de la norme de prononciation québécoise. Un résultat secondaire peut par ailleurs être relevé : comme l'a également montré 
l'analyse des productions des locuteurs-modèles, le déterminant un semble légèrement plus susceptible d'être réalisé comme un [ $\tilde{\varepsilon}]$. Cette tendance est cependant très marginale et ne repose que sur les résultats liés au stimulus syntagmatique, moins fiables. Elle peut donc seulement être qualifiée d'hypothétique et devra être vérifiée dans de futures enquêtes similaires.

\subsubsection{Réalisation de $/ \tilde{a} /:[\tilde{\mathbf{e}}] \sim[\tilde{a}] /[\tilde{\mathbf{b}}]$}

Le deuxième trait touchant à des voyelles nasales testé perceptivement est la réalisation phonétique de la voyelle nasale /ã/. Parmi les trois réalisations phonétiques codées dans les productions des locuteurs-modèles $([\tilde{\dddot{x}}] /[\tilde{a}] \sim[\tilde{e}]$ $\sim[\tilde{a}] /[\tilde{b}])$, une forte domination du [ẽ] central a pu être observée, et ce, indépendamment du contexte linguistique. Par ailleurs, alors que ces résultats ont

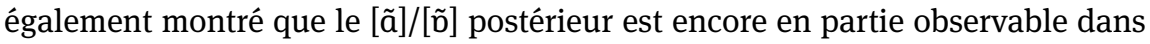
l'usage de deux locuteurs, le [ã]/[ã] antérieur s'est avéré quasiment inexistant (cf. 5.3.2.2). C'est la raison pour laquelle les tests de perception sur la base desquels ces hypothèses seront vérifiées se basent non pas sur les trois variantes

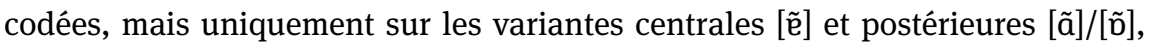
aucun stimulus adéquat n'ayant pu être trouvé pour la réalisation antérieure

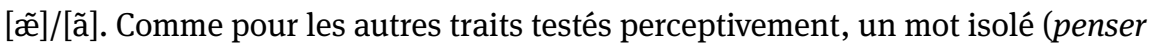
[pẽse] [pãse] ; liste de mots PFC) et un mot intégré dans un syntagme (vendue dans l'ancienne poste vendue [vẽdy] [vãdy] ; texte PFC) ont été pris en compte.

\section{Question 1 : évaluation (sans contexte explicite)}

Les résultats de la première question portant sur le mot isolé penser montrent une tendance similaire à l'analyse des productions. La variante centrale [ẽ ] est en effet préférée par la majorité des informateurs : elle obtient un score moyen de 93,58\% (écart-type : 9,85\%), contre 84,70\% (écart-type : 23,32\%) pour la variante postérieure $[\tilde{a}] /[\tilde{o}]$, cette différence étant hautement significative $(p<0,001)$ (cf. figure 170).

Soulignons que l'analyse de variance indique également un léger effet significatif du sexe sur l'évaluation perceptive de la variante postérieure [ã]/[ñ] $(F=$ $5,55, p<0,05)$ : en moyenne, les femmes semblent en effet évaluer cette variante de manière nettement plus positive que les hommes. Étant donnée la tendance des femmes, bien connue en sociolinguistique, à se tourner davantage vers les variantes standards, ce résultat pourrait suggérer que la variante postérieure présente encore un certain niveau de prestige manifeste dans les attitudes des informateurs, même s’il reste inférieur à celui de la variante centrale [ẽ]. 
Évaluation de $[\tilde{a}] /[\tilde{\mathfrak{v}}]$ vs $[\tilde{\mathrm{e}}]$ dans penser (liste de mots PFC)

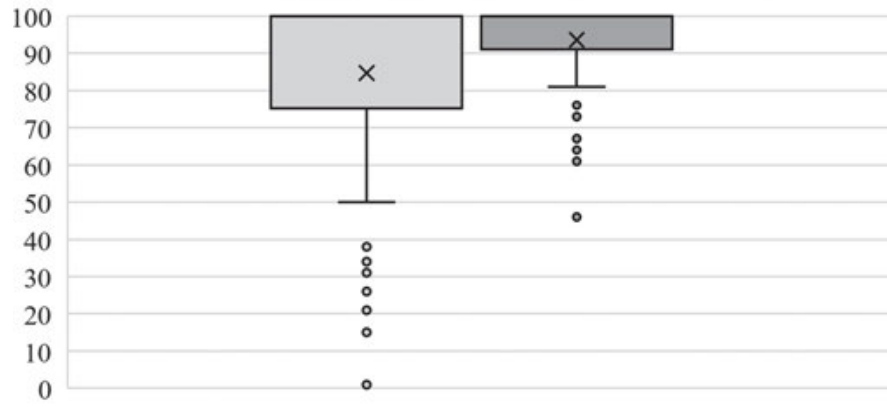

\section{$[\tilde{\mathrm{a}}] /[\tilde{\mathrm{v}}] \quad \square[\tilde{\mathrm{e}}]$}

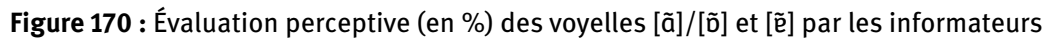
québécois $(\mathrm{n}=96)$ dans le mot isolé <penser> (ANOVA : $p<0,001)$.

Le résultat du syntagme l'ancienne poste vendue montre une tendance similaire en faveur du stimulus avec variante centrale [ẽ], moins forte néanmoins que dans le cas du mot isolé penser : le [ẽ ] obtient en effet un score moyen de $88,91 \%$ (écart-type : 15,38\%), significativement plus élevé $(p<0,05)$ que le score moyen de 83,83\% (écart-type : 18,34\%) du [ã]/[õ] (cf. figure 171).

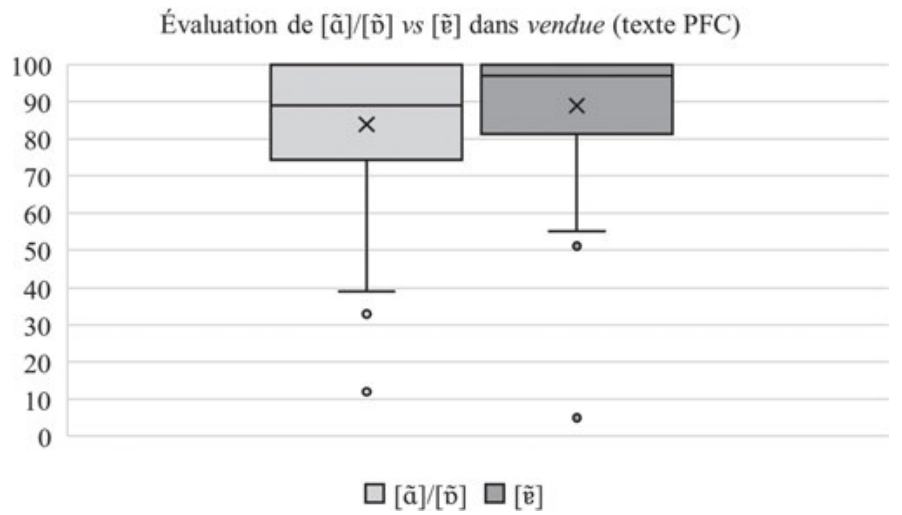

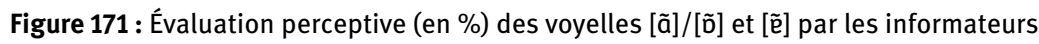
québécois $(\mathrm{n}=96)$ dans le mot $<$ vendue $>$ tiré du texte $\operatorname{PFC}($ ANOVA : $p<0,05)$.

Un effet du niveau d'éducation des informateurs sur l'évaluation des deux stimuli est également relevé par l'analyse de variance : d'une part, ceux dont le niveau d'éducation est plus élevé évaluent le [ã]/[ñ] plus positivement que les moins 
diplômés $(F=8,77, p<0,01) .^{2}$ D'autre part, dans le sens inverse, ces derniers évaluent plus positivement la variante antérieure [ẽ] que les informateurs ayant un niveau d'éducation plus élevé $(F=5,59, p<0,05)$. Cette différence de comportement chez les informateurs selon leur niveau d'éducation est très similaire aux différences trouvées dans les évaluations du mot isolé en fonction du sexe (cf. supra). Or, étant donné que les informateurs plus diplômés présentent généralement - tout comme les femmes (cf. supra) - une tendance à privilégier les variantes standards, cette différence pourrait renforcer l'interprétation selon laquelle la variante postérieure $[\tilde{a}] /[\tilde{o}]$ aurait gardé un certain degré de prestige manifeste. Mentionnons finalement que les différents degrés dans la tendance en faveur du [ẽ] relevés dans ces deux questions sont vraisemblablement également explicables par le problème méthodologique de la longueur du stimulus syntagmatique, qui tend à biaiser les résultats des évaluations.

\section{Question 2 : prononciation idéale des présentateurs de journaux télévisés}

Les résultats de la question portant sur la prononciation idéale pour les présentateurs de télévision à propos du mot isolé penser présentent des résultats très

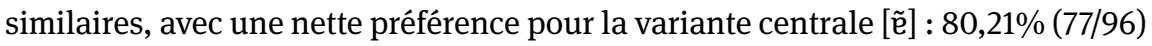
des informateurs la préfèrent en effet à la variante postérieure [ã]/[0̃], choisie par $16,67 \%$ (16/96) seulement d'entre eux. Étant donnée la clarté de ces résultats, il n'est pas étonnant que cette différence soit hautement significative $(p<0,001)$ (cf. figure 172).

Le syntagme l'ancienne poste vendue suscite à nouveau des tendances similaires bien que moins nettes, 53,13\% des informateurs se prononçant pour la

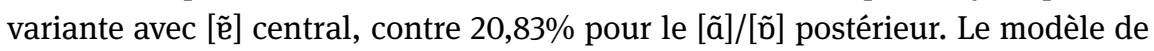
régression montre, pour sa part, que cette différence de taux est significative à $p<0,001$ (cf. figure 173).

Les résultats de cette question confirment ainsi globalement les tendances déjà mises en lumière dans la première, à savoir la large préférence des informateurs québécois pour la variante centrale [ẽ $]$. Au contraire de la première question, cependant, aucun effet du sexe ou de l'éducation des informateurs n'a pu être relevé $(p>0,05)$.

2 Rappelons que tout informateur titulaire soit d'un diplôme universitaire, soit d'un diplôme d'études collégiales (DEC) des programmes d'études pré-universitaires est classé dans la catégorie des informateurs ayant un niveau d'éducation plus élevé, alors que les détenteurs d'un diplôme du primaire, du secondaire, d'une école de métiers ou d'un diplôme d'études collégiales (DEC) sont considérés comme des informateurs ayant un niveau d'éducation moins élevé (cf. 3.3.2). 
Selon vous, comment un présentateur de télévision québécois devrait-il prononcer le mot suivant ? «penser»

100
90
80
70
60
50
40
30
20
10
0

00

90

80

70

60

50

40

30

20

10

0

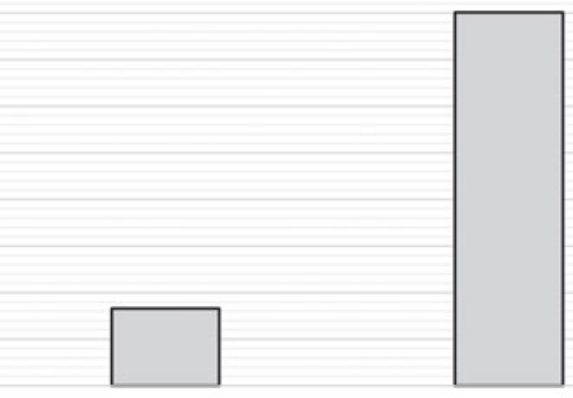

Variante avec $[\tilde{a}] /[\tilde{b}]$

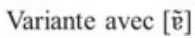

Sans réponse

Figure 172 : Voyelle privilégiée (en $\%$ ) dans le mot <penser> pour les présentations de journaux télévisés $(\mathrm{n}=96)$ - Mots isolés ( $p<0,001$, coef. $=1,70$, e.s. $=0,20, t=8,38)$.

Selon vous, comment un présentateur de télévision québécois devrait-il prononcer le groupe de mots suivant? « vendue »

100

90

80

70

60

50

40

30

20

10

0

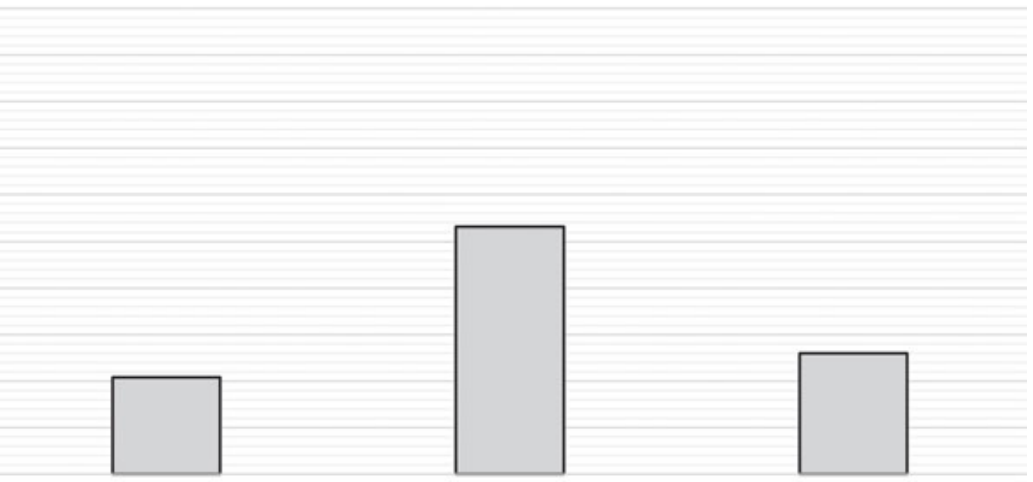

Variante avec $[\tilde{\mathrm{a}}] /[\tilde{\mathrm{v}}]$

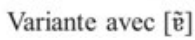

Sans réponse

Figure 173 : Voyelle privilégiée (en \%) dans le mot <vendue> pour les présentations de journaux télévisés $(\mathrm{n}=96)$ - Texte PFC $(p<0,001$, coef. $=1,85$, e.s. $=0,38, t=4,85)$.

\section{Question 3 : prononciation à enseigner dans les cours de français langue étrangère (FLE)}

Les mêmes tendances sont également observables dans les réponses des informateurs à la question sur la prononciation-cible en FLE par rapport au mot isolé penser : une large majorité de 69,79\% (67/96) d'entre eux préfère en effet la variante 
Selon vous, laquelle des deux prononciations du mot suivant serait la plus adaptée à être enseignée à des non-francophones au

Québec ? «penser»

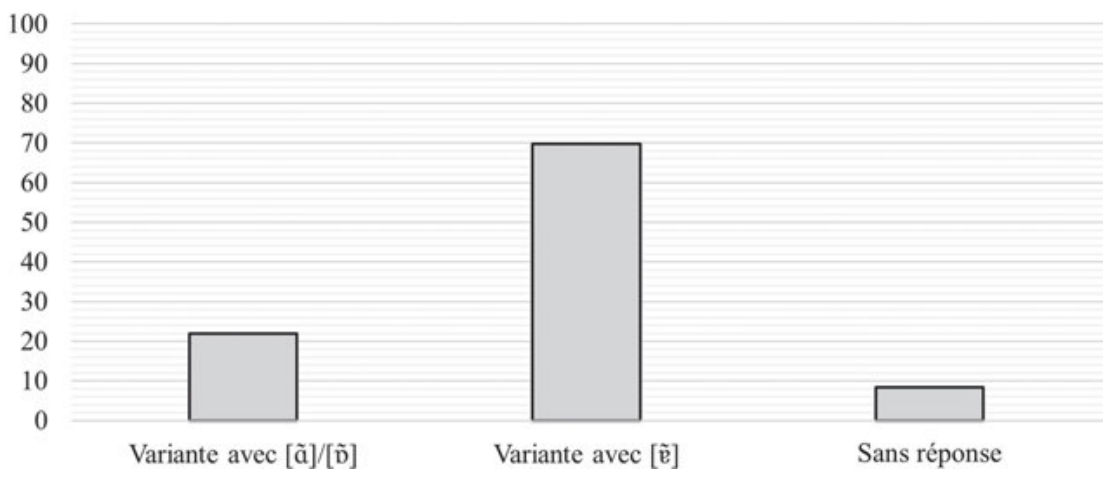

Figure 174 : Voyelle privilégiée (en \%) dans le mot <penser> pour les cours de français langue étrangère $(\mathrm{FLE})(\mathrm{n}=96)$ - Mots isolés ( $p<0,001$, coef. $=2,72$, e.s. $=0,36, t=7,65)$.

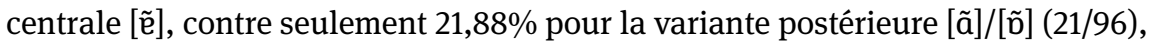
cette différence de taux étant hautement significative $(p<0,001)$ (cf. figure 174).

Le résultat est très similaire pour le syntagme l'ancienne poste vendue, bien qu'il soit ici aussi moins univoque, avec une variante centrale [ẽ préférée par 55,21\% (53/96) des informateurs et une variante postérieure [ã]/[õ] choisie par 23,96\% (23/96) d'entre eux, cette différence étant à nouveau significative à $p<0,001$ (cf. figure 175).

Selon vous, laquelle des deux prononciations du groupe de mots suivant serait la plus adaptée à être enseignée à des non-francophones au Québec? «L'ancienne poste vendue »

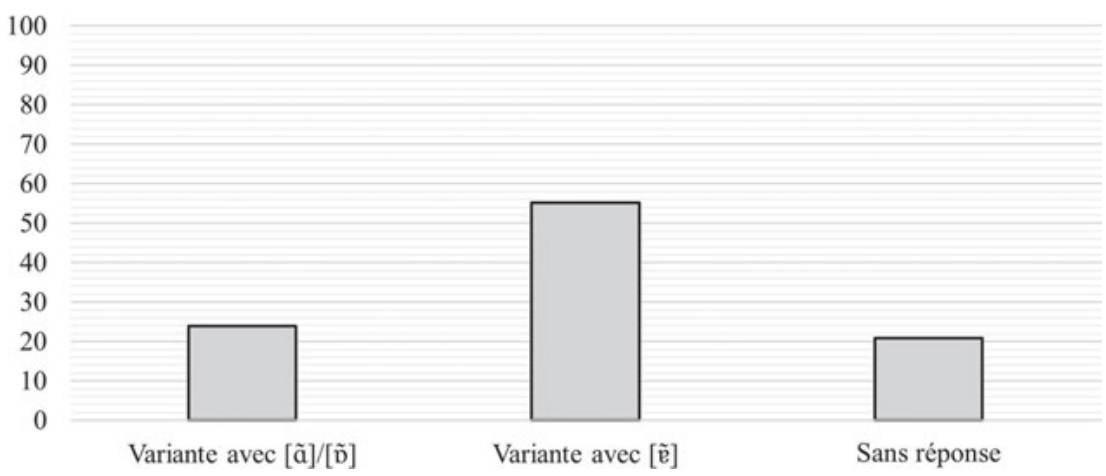

Figure 175 : Voyelle privilégiée (en \%) dans le mot <vendue> pour les cours de français langue étrangère (FLE) $(\mathrm{n}=96)$ - Texte $\mathrm{PFC}(p<0,001$, coef. $=2,13$, e.s. $=0,32, t=6,69)$. 
Notons qu'aussi bien dans le cas du mot isolé penser (cf. figure 174) que dans celui du syntagme vendue (cf. figure 175), le modèle de régression ne montre aucun effet significatif des variables sociodémographiques ( $p>0,05)$. Les résultats de cette question ne font ainsi que confirmer les tendances des deux précédentes, avec une variante centrale [ẽ] manifestement largement préférée par les informateurs québécois.

En conclusion, deux observations peuvent être retenues de ces résultats : premièrement, indépendamment du type de norme pris en compte, la tendance globale est largement en faveur de la variante centrale [ẽ], ce qui confirme les résultats de l'analyse des productions des locuteurs-modèles, ces derniers faisant majoritairement usage de cette même variante (cf. 5.3.2.2). C'est donc celle-ci qui semble convenir le mieux pour la définition de la norme de prononciation qué-

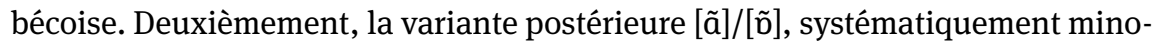
ritaire dans les trois questions, semble tout de même encore présenter un certain prestige manifeste, parallèle à celui de la variante centrale [ẽ]. C'est du moins ce que suggèrent les effets significatifs du sexe et de l'éducation relevés dans le cadre de la première question (cf. supra), les femmes et les informatrices et informateurs plus diplômés ayant tendance à les évaluer de manière bien plus positive que leurs homologues masculins et de niveau d'éducation plus faible. Il resterait cependant à vérifier ce résultat secondaire dans de futures enquêtes, car il ne se base que sur les données recueillies dans la première question.

\subsubsection{Réalisation de $/ \tilde{\varepsilon} /:[\tilde{e}] \sim[\tilde{\varepsilon}]$}

Le dernier trait touchant à des voyelles nasales ayant été testé dans le cadre du test de perception effectué au Québec concerne la réalisation phonétique de la voyelle nasale / $\tilde{\varepsilon} /$. Rappelons ce faisant que l'analyse des productions a montré que la réalisation très largement majoritaire dans l'usage des locuteurs-modèles est le [ẽ] antérieur. Ce résultat, qui confirme a priori le consensus des études publiées auparavant (cf. Cox 1998 ; Reinke 2005 ; Bigot/Papen 2013 ; Côté 2016), sera confronté ici aux données de perception à l'exemple de deux contextes : le mot isolé médecin (liste de mots PFC) et le mot coin, testé dans le syntagme dans le coin (texte PFC). Il faut cependant noter que ces deux cas ne sont pas totalement comparables étant donné leur contexte linguistique différent : en effet, alors que la voyelle / $\tilde{\varepsilon} /$ du syntagme dans le coin fait partie d'une diphtongue (elle est précédée de la glissante [w]), le / $\tilde{\varepsilon} /$ de médecin se trouve dans une monophtongue précédée de la fricative [s]. Cette différence devra donc être prise en compte dans l'interprétation des données. 


\section{Question 1 : évaluation (sans contexte explicite)}

Les résultats obtenus tout d'abord par l'évaluation des deux variantes du mot isolé médecin sans mention explicite de quelque contexte que ce soit montrent, conformément à ceux des productions des locuteurs-modèles, une préférence des informateurs pour la variante antérieure [ẽ] : cette dernière obtient en effet un score de $89,85 \%$ (écart-type : 15,42\%), contre $82,31 \%$ (écart-type : $22,60 \%$ ) pour la variante postérieure $[\tilde{\varepsilon}]$, cette différence étant significative à $p<0,01$. Signalons

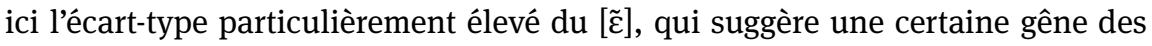
informateurs par rapport à cette variante, ce qui renforce l'interprétation allant en faveur de la variante antérieure [ẽ] (cf. figure 176).

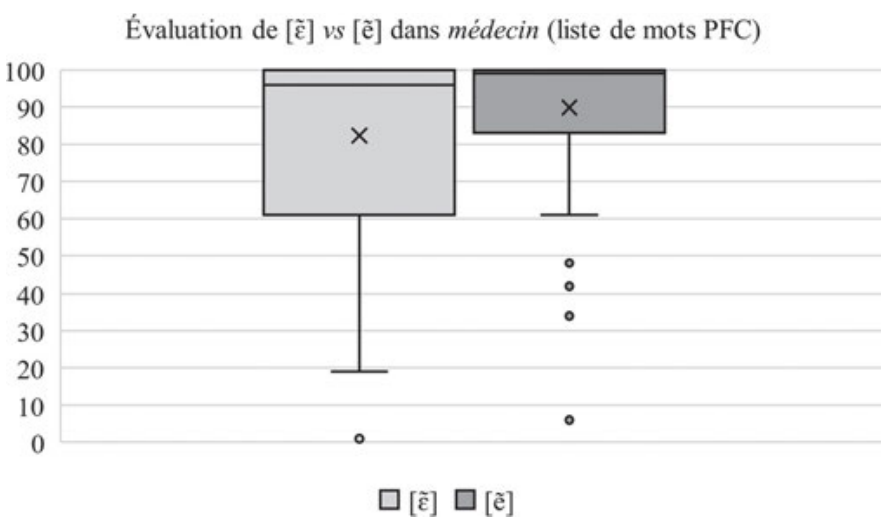

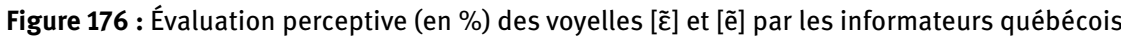
$(\mathrm{n}=96)$ dans le mot isolé <médecin> $($ ANOVA : $p<0,01)$.

Les évaluations des variantes du stimulus syntagmatique dans le coin indiquent également une légère préférence pour la variante antérieure [ẽ]. L'analyse de variance indique cependant qu'au contraire du cas du mot isolé médecin, la différence entre les scores obtenus par chacune des variantes n'est pas significative $(p>0,05)$. Par ailleurs, l'analyse de variance ne montre pas non plus d'effet significatif des facteurs sociodémographiques (cf. figure 177).

Labsence de signification statistique de ce deuxième résultat est vraisemblablement attribuable à deux effets : premièrement, comme ailleurs dans le présent test de perception, il pourrait s'agir d'un effet de la longueur du stimulus syntagmatique rendant son évaluation plus difficile et moins univoque. Deuxièmement, le contexte linguistique pourrait, comme mentionné plus haut, avoir joué un rôle : la voyelle / $\tilde{\varepsilon} /$ à évaluer dans le syntagme dans le coin est en effet précédée de la glissante [w], ce qui n'est pas le cas dans le mot isolé médecin, dont le contexte à gauche $\mathrm{du}[\tilde{\varepsilon}]$ est la fricative $[\mathrm{s}]$. Or, au niveau acoustique, il est 


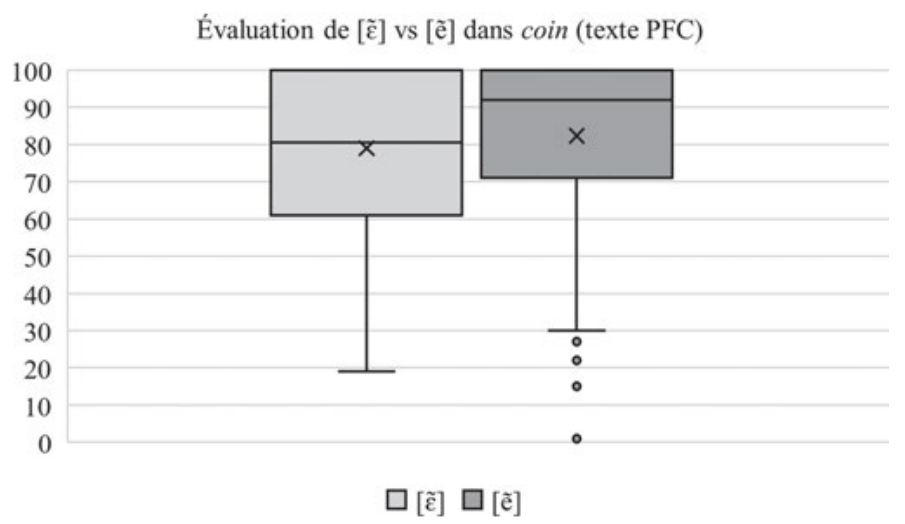

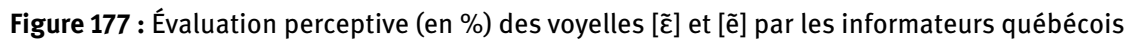
$(\mathrm{n}=96)$ dans le mot $<$ coin> tiré du texte PFC (ANOVA : $p>0,05)$.

impossible de déterminer avec certitude la fin d'une glissante et le début de la voyelle qui suit, étant données l'instabilité et l'articulation constamment en mouvement des glissantes (cf. Meunier 2007, 169) ; il est donc possible que l'évaluation perceptive du [ $\tilde{\varepsilon}]$ ait été considérablement biaisée par la glissante [w] qui la précède. Les résultats du stimulus syntagmatique doivent dès lors être interprétés avec une grande précaution et ne semblent pas aussi fiables que ceux du mot isolé médecin. La tendance globale de cette première question est de privilégier la variante endogène [ẽ].

\section{Question 2 : prononciation idéale des présentateurs de journaux télévisés}

En ce qui concerne la prononciation à recommander dans les journaux télévisés pour le mot isolé médecin, les réponses confirment tout d'abord très largement la préférence pour la variante antérieure [ẽ] : cette dernière est en effet choisie par une très large majorité de 85,42\% (82/96) des informateurs, alors que seuls 10,42\%

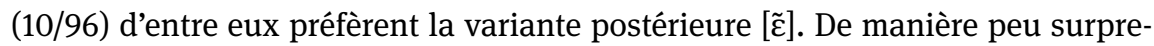
nante étant donnée la clarté de ces résultats, le modèle de régression indique que cette différence de taux est hautement significative $(p<0,001)$ (cf. figure 178).

La tendance est similaire pour le stimulus syntagmatique dans le coin, bien que moins affirmée : 60,42\% (58/96) des informateurs préfèrent la variante antérieure [ẽ], alors que 32,29\% (31/96) préfèrent la variante postérieure [̃̃]. Le modèle indique, ici aussi, que la différence entre ces taux est significative à $p<0,001$, et met également en évidence un effet significatif du sexe $(p<0,05)$ : les femmes

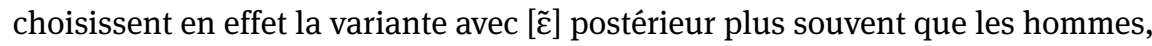
même si en moyenne, leur préférence va également plutôt vers le [ẽ] antérieur (cf. figure 179). 
Selon vous, comment un présentateur de télévision québécois devrait-il prononcer le mot suivant?

« médecin »

100

90

80

70

60

50

40

30

20

10

0

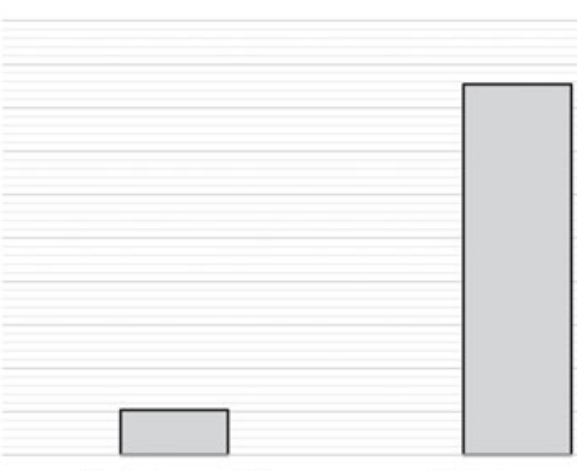

Variante avec $[\tilde{\varepsilon}]$

Variante avec [ẽ]

Sans réponse

Figure 178 : Voyelle privilégiée (en \%) dans le mot <médecin> pour les présentations de journaux télévisés $(n=96)$ - Mots isolés $(p<0,001$, coef. $=2,15$, e.s. $=0,18, t=12,04)$.

Selon vous, comment un présentateur de télévision québécois devrait-il prononcer le groupe de mots suivant ? « dans le coin »

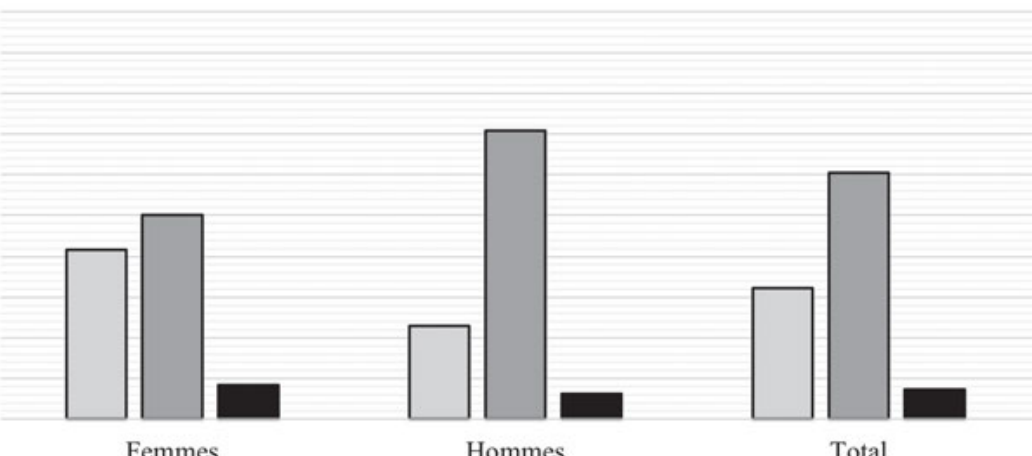

Femmes

Hommes

Total

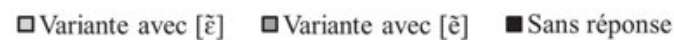

Figure 179 : Voyelle privilégiée (en \%) dans le mot <coin> pour les présentations de journaux télévisés $(\mathrm{n}=96)$ - Texte PFC ; réponses présentées dans leur globalité $(p<0,001$, coef. $=1,80$, e.s. $=0,27, t=6,80$ ) ainsi que selon le sexe des informateurs ( $p<0,05$, coef. $=0,22$, e.s. $=0,10$, $t=2,08)$.

Mise à part la confirmation globale de la préférence pour la variante antérieure [ẽ], une différence est donc observable selon le sexe. Or, selon les études sociolinguistiques mentionnées précédemment (cf. entre autres Labov 1963 ; Rovere 1982 ; Bourdieu 1982 ; cf. 2.1.2.2), les femmes ont tendance, on l'a dit, à privilégier les variantes possédant un prestige manifeste. Dès lors, à l'instar du 


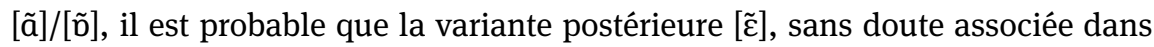
leurs attitudes au « français parisien », détienne encore un certain prestige manifeste, parallèlement à celui du [ẽ], plus considérable encore.

\section{Question 3 : prononciation à enseigner dans les cours de français langue étrangère (FLE)}

Les réponses des informateurs à la question sur la prononciation à privilégier dans les cours de FLE confirment aussi bien la préférence générale pour le [ẽ] antérieur que l'effet du sexe. En effet, d'une part, 88,54\% (85/96) des informateurs privilégient le [ẽ], $11,46 \%$ (11/96) seulement préférant le [च̃], et cette différence est hautement significative $(p<0,001)$. D'autre part, le modèle de régression montre également un effet significatif du sexe $(p<0,01)$, les femmes choisissant plus souvent le $[\tilde{\varepsilon}]$ postérieur $(20,83 \%, 10 / 48)$ que les hommes $(2,08 \%, 1 / 48)$, même si globalement elles préfèrent également largement plus souvent le [ẽ] antérieur (79,17\%, 38/48) (cf. figure 180).

\section{Selon vous, laquelle des deux prononciations du mot suivant serait la plus adaptée à être enseignée à des non-francophones au Québec ? \\ " médecin "}

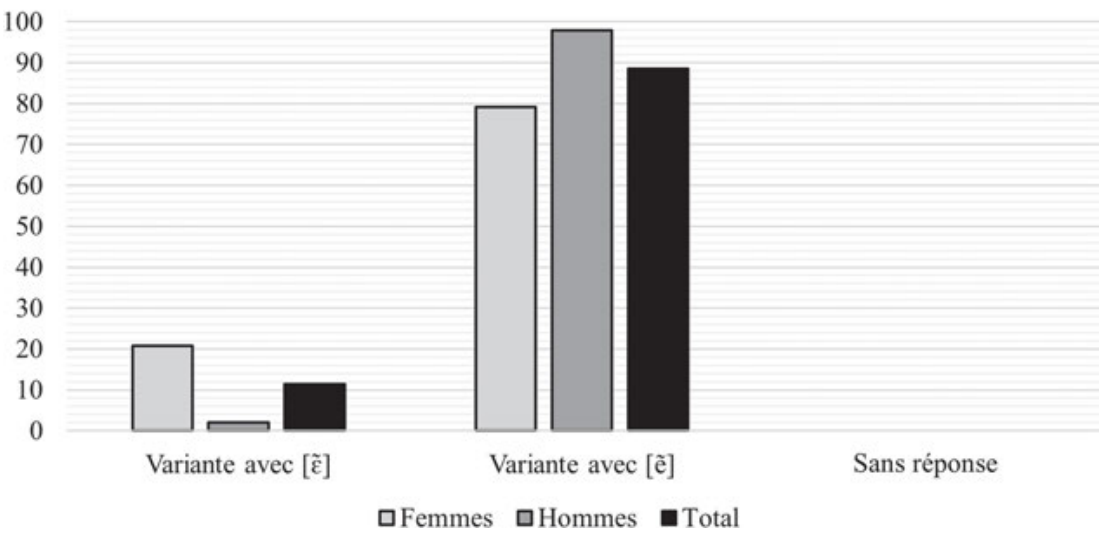

Figure 180 : Variante privilégiée (en \%) dans le mot <médecin> pour les cours de français langue étrangère (FLE) $(\mathrm{n}=96)$ - Liste de mots PFC ; réponses présentées dans leur globalité $(p<0,001$, coef. $=1,44$, e.s. $=0,14, t=10,53)$ ainsi que selon le sexe des informateurs $(p<0,01$, coef. $=0,14$, e.s. $=0,05, t=2,68$ ).

L'interprétation proposée dans le cas de la question portant sur les présentateurs de télévision (cf. supra) se confirme donc ici : les femmes ayant plus tendance que les hommes à préférer les variantes possédant un prestige manifeste, il est possible que ce résultat soit l'indice d'un certain degré de prestige manifeste conservé 
par la variante postérieure $[\tilde{\varepsilon}]$ parallèlement à celui, bien établi et stable, de la variante antérieure [ẽ].

Finalement, cette même question appliquée au stimulus syntagmatique dans le coin induit les mêmes tendances globales, même si, comme souvent dans le cas des stimuli syntagmatiques du présent test de perception, celles-ci sont moins univoques que les résultats du mot isolé : 62,50\% (60/92) des informateurs privilégient en effet la variante antérieure [ẽ], alors que 29,17\% (28/96) portent leur choix sur la variante postérieure $[\tilde{\varepsilon}]$, cette différence étant hautement significative $(p<0,001)$. L'analyse de régression ne montre cependant, au contraire du cas du mot isolé, aucun effet du sexe sur les résultats ( $p>0,05)$ (cf. figure 181).

Selon vous, laquelle des deux prononciations du groupe de mots suivant serait la plus adaptée à être enseignée à des non-francophones au

Québec? «dans le coin »

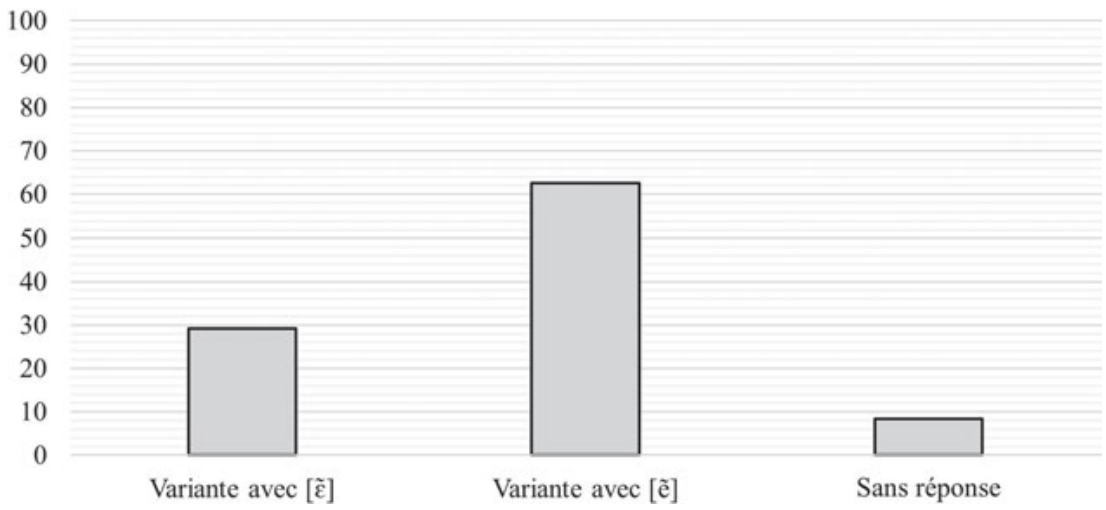

Figure 181 : Voyelle privilégiée (en \%) dans le mot <coin> pour les cours de français langue étrangère (FLE) $(\mathrm{n}=96)$ - Texte PFC ( $p<0,001$, coef. $=1,71$, e.s. $=0,31, t=5,50)$.

Dans l'optique du présent ouvrage, deux conclusions peuvent être ainsi tirées de ces trois questions touchant à la réalisation phonétique $\mathrm{du} / \tilde{\varepsilon} /$ : tout d'abord, la variante antérieure [ẽ] est quasiment systématiquement préférée par les informateurs québécois, et ce, indépendamment de la question ou du type de stimulus utilisés. Étant donné que cette variante est également largement majoritaire dans les productions des locuteurs-modèles québécois, elle semble être la plus adéquate dans l'optique de la définition d'une norme de prononciation québécoise. Cependant, un résultat secondaire peut également être relevé par rapport à la variante postérieure $[\tilde{\varepsilon}]$ : les effets de la variable du sexe sur deux des questions traitées dans le présent sous-chapitre semblent en effet montrer que cette variante continue à présenter un certain prestige manifeste, parallèlement à celui, très stable, du [ẽ] antérieur. 


\subsubsection{Discussion}

Dans le cadre du présent sous-chapitre, un total de neuf traits québécois potentiellement standards ont été soumis à des évaluations perceptives auprès d'un échantillon de 96 informateurs sélectionnés sur la base de quotas prenant en compte l'origine des informateurs, leur sexe, leur âge et leur niveau d'éducation (cf. 3.3.2). Les traits ainsi testés sont identiques aux traits analysés dans les productions de locuteurs-modèles du chapitre précédent (cf. 5.3). Afin de permettre une estimation de l'appartenance (ou non) de ces neuf traits à la norme de prononciation québécoise, les résultats des tests de perception ne sont pas seulement résumés dans le tableau 77, mais ils y sont également comparés aux résultats de ces analyses de production.

Tableau 77 : Comparaison des résultats touchant aux productions et perceptions pour les neuf traits étudiés dans le cas du Québec.

\begin{tabular}{|c|c|c|c|c|}
\hline $\begin{array}{l}\text { Traits de } \\
\text { prononciation } \\
\text { québécois }\end{array}$ & Contextes testés & Productions & Perceptions & Commentaires \\
\hline $\begin{array}{l}\text { 1. Opposition } \\
/ \mathrm{a} / \mathrm{a} / \mathrm{a} / \mathrm{l}\end{array}$ & $\begin{array}{l}\text { patte /pat/ } \\
\text { pâate /pat:/ }\end{array}$ & /a/: /a:/ & & I \\
\hline $\begin{array}{l}\text { 2. /a/ final dans } \\
\text { <-oi> en tant } \\
\text { que [wa] }\end{array}$ & $\begin{array}{l}\text { baignoire } \\
\text { [benwar], trois } \\
\text { [tвwa] }\end{array}$ & [wa] & & $\begin{array}{l}\text { Généralisation des } \\
\text { contextes testés } \\
\text { impossible étant } \\
\text { donnée la grande } \\
\text { variation lexicale }\end{array}$ \\
\hline $\begin{array}{l}\text { 3. Opposition } \\
\text { quantitative } \\
\text { / } / \text { / vs. / } \varepsilon \text { : }\end{array}$ & $\begin{array}{l}\text { faites } / \varepsilon / \sim \text { fête } \\
/ \varepsilon: /\end{array}$ & \multirow{2}{*}{\multicolumn{2}{|c|}{$\begin{array}{l}\mid \varepsilon /: / \varepsilon: / \sim \\
\left|a^{3}\right|\end{array}$}} & $\begin{array}{l}\text { Perceptions: } \\
-\quad / \varepsilon /: / \varepsilon: / \text { : prestige } \\
\quad \text { manifeste }\end{array}$ \\
\hline $\begin{array}{l}\text { 4. Diphtongaison } \\
\text { de la voyelle } \\
\text { allongée / } \varepsilon \text { / }\end{array}$ & fête / $\mathrm{fa}^{3} \mathrm{t} /$ & & & $\begin{aligned}- & \mid \varepsilon /: / a^{3} /: \text { prestige } \\
& \text { latent }\end{aligned}$ \\
\hline $\begin{array}{l}\text { 5elâchement } \\
\text { des voyelles } \\
\text { /i, y, u/ en } \\
\text { finale de } \\
\text { mot devant } \\
\text { consonne non } \\
\text { allongeante }\end{array}$ & $\begin{array}{l}\text { sịx [sıs], } \\
\text { villes [vıl] }\end{array}$ & [I] (et [r], [v]) & elâchés) & 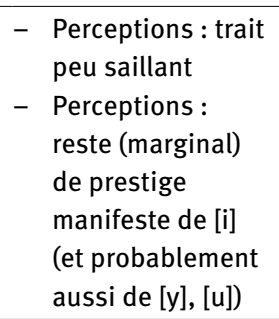 \\
\hline
\end{tabular}




\begin{tabular}{|c|c|c|c|}
\hline $\begin{array}{l}\text { Traits de } \\
\text { prononciation } \\
\text { québécois }\end{array}$ & Contextes testés & Productions & Commentaires \\
\hline $\begin{array}{l}\text { 6. Dévoisement } \\
\text { ou effacement } \\
\text { des voyelles } \\
\text { /i, y, u/ entre } \\
\text { consonnes } \\
\text { sourdes }\end{array}$ & 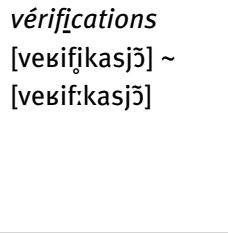 & $\begin{array}{l}\text { Pas de dévoisement/ } \\
\text { d'effacement }\end{array}$ & $\begin{array}{l}\text { Perceptions : } \\
\text { - /i/ voisé : prestige } \\
\text { manifeste } \\
\text { - } \text { /i/ syncopé : } \\
\text { prestige latent }\end{array}$ \\
\hline $\begin{array}{l}\text { 7. Opposition } \\
/ \tilde{œ} /: / \tilde{\varepsilon} /\end{array}$ & 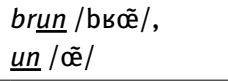 & / œ̃/: / $\tilde{\varepsilon} /$ & $\begin{array}{l}{[\tilde{\varepsilon}] \text { marginalement }} \\
\text { admissible dans un }\end{array}$ \\
\hline 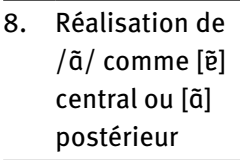 & $\begin{array}{l}\text { penser [pẽse], } \\
\text { vendue [vẽdzy] }\end{array}$ & [ẽ] & $\begin{array}{l}\text { Reste (marginal) de } \\
\text { prestige manifeste } \\
\text { du [ã] }\end{array}$ \\
\hline $\begin{array}{l}\text { 9. Réalisation de } \\
/ \tilde{\varepsilon} / \text { comme [ẽ] } \\
\text { antérieur }\end{array}$ & $\begin{array}{l}\text { médecin } \\
\text { [medsẽ], } \\
\text { coịn [kwẽ] }\end{array}$ & [ẽ] & $\begin{array}{l}\text { Reste (marginal) de } \\
\text { prestige manifeste } \\
\text { du }[\tilde{\varepsilon}]\end{array}$ \\
\hline
\end{tabular}

Notons que, contrairement au cas de la Suisse romande, qui présente une norme actuellement évoluant en direction de la norme parisienne, la norme de prononciation québécoise semble globalement bien établie et stable. Malgré cet état de fait, une certaine ambiguïté peut être observée dans les attitudes des informateurs face à la variante exogène de certains traits, généralement associée par eux à la norme " parisienne » : un prestige manifeste de certaines variantes exogènes semble en effet continuer à exister dans les attitudes des informateurs québécois - en particulier dans le cas des traits 5, 6, 8 et 9 (cf. tableau 77). Or, on observe que les quatre traits concernés par cette ambiguïté représentent tous des variantes phonétiques, alors que dans le cas d'oppositions phonémiques, les évaluations sont plus univoques (cf. tableau 77 : traits 1, 4, 7). Ainsi, les oppositions phonémiques sembleraient être plus stables dans la norme de prononciation québécoise que les différences de nature phonétique.

Une réduction schématique de la complexité de ces résultats aux points essentiels montre à présent que sur les neuf traits endogènes étudiés, sept peuvent être considérés comme faisant partie de la norme de prononciation québécoise, alors que deux n'en font pas partie : dans le cas du dévoisement/ de l'effacement des voyelles $/ \mathrm{i}, \mathrm{y}, \mathrm{u} /$, en raison de résultats montrant une préférence pour les variantes exogènes voisées $/ \mathrm{i}, \mathrm{y}, \mathrm{u}$ /, et pour la diphtongaison de la voyelle allongée $/ \varepsilon:$, parce que les résultats des productions et des perceptions 
ne sont pas univoques et doivent donc encore faire l'objet d'analyses plus poussées (cf. tableau 78).

Tableau 78 : Résumé schématique des traits appartenant (ou non) à la norme de prononciation québécoise.

\begin{tabular}{|c|c|c|}
\hline \multicolumn{3}{|c|}{ Traits faisant partie de la norme de prononciation québécoise } \\
\hline Trait & \multicolumn{2}{|l|}{ Contextes testés } \\
\hline Opposition /a/: /a:/ & \multicolumn{2}{|c|}{ pátte /pat/ pâte /pait/ } \\
\hline /a/ final dans <-oi> réalisé en tant que [wa] & \multicolumn{2}{|c|}{ baignoire [вعлwaв], trois [tьwa] } \\
\hline Opposition quantitative $/ \varepsilon /: / \varepsilon: /$ & \multicolumn{2}{|c|}{ faites / $\varepsilon / \sim$ fête $/ \varepsilon: /$} \\
\hline $\begin{array}{l}{[\mathrm{I}],[\mathrm{Y}],[\mho] \text { relâchés en finale de mot devant }} \\
\text { consonne non allongeante }\end{array}$ & \multicolumn{2}{|l|}{ sịx [sıs], villes [vıl] } \\
\hline Opposition / $\tilde{e} /: / \tilde{\varepsilon} /$ & \multicolumn{2}{|l|}{ brun / bьø̃ /, un / ̃̃)/ } \\
\hline Réalisation de /ã / comme [ẽ] central & \multicolumn{2}{|c|}{ p. ex. penser [pẽse], vendue [vẽdzy] } \\
\hline Réalisation de / $\tilde{\varepsilon} /$ comme [ẽ] antérieur & \multicolumn{2}{|c|}{ p. ex. médecin [medsẽ], coin [kwẽ] } \\
\hline \multicolumn{3}{|c|}{ Traits ne faisant a priori pas partie de la norme de prononciation québécoise } \\
\hline Trait & Contextes testés & Justification \\
\hline Diphtongaison de la voyelle $/ \varepsilon: /$ en $\left[a^{3}\right]$ & fête / $\mathrm{fa}^{3} \mathrm{t} /$ & Préférence pour le / $\varepsilon: /$ \\
\hline $\begin{array}{l}\text { Dévoisement/effacement des voyelles } \\
/ \mathrm{i}, \mathrm{y}, \mathrm{u} / \text { entre consonnes sourdes }\end{array}$ & 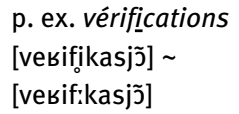 & $\begin{array}{l}\text { Productions et perceptions } \\
\text { non univoques }\end{array}$ \\
\hline
\end{tabular}

Ainsi, contrairement à la Suisse romande, pour laquelle j'ai pu montrer que la majorité des traits se trouvent actuellement dans une phase de changement allant en direction de la norme du français de référence (cf. 6.2.6), la norme québécoise présente - dans les productions de locuteurs-modèles et dans les perceptions d'une majorité des auditeurs - des traits endogènes bien établis et stables. Il est ainsi frappant que les résultats touchant aux représentations et attitudes (cf. 4.4.2), aux productions (cf. 5.3.3) et aux perceptions (cf. 6.3) confirment unanimement l'existence d'une norme de prononciation québécoise bien établie. Au contraire du cas de la Suisse romande, il semble donc tout à fait pertinent de postuler l'existence d'une telle norme.

\subsection{Problèmes méthodologiques récurrents}

Finalement, quelques réserves doivent être formulées par rapport aux stimuli utilisés dans les trois tests de perception : tout d'abord, les résultats liés aux syntagmes extraits du texte PFC dénotent dans l'ensemble chez les informateurs une 
certaine hésitation quant au trait à évaluer, éventuellement due à la longueur des stimuli. Il est en effet possible que pour les informateurs, celle-ci ait compliqué le discernement du trait à évaluer, ce qui fait par ailleurs ressortir un degré de saillance relativement faible des traits testés. On observe en outre, principalement pour les syntagmes extraits du texte PFC, que les informateurs présentent globalement une forte préférence pour les productions des locuteurs-modèles par rapport à celles des locuteurs ordinaires, indépendamment de l'élément testé. Cette observation est particulièrement valable pour les résultats du test de perception à Paris (cf. 6.1). Or, il est probable dans ce cas-ci que les auditeurs non experts, au lieu d'évaluer le trait à tester, aient jugé la production globale des locuteurs-modèles, en tentant d'estimer si celle-ci correspond à leurs attentes en matière, d'une part, de compétences en lecture à haute voix chez un professionnel de la parole et, d'autre part, de leur rythme particulier en lecture. Léon (1993) soulignait déjà les particularités rythmiques et accentuelles de la lecture à haute voix des présentateurs des informations :

« Le phonostyle des nouvelles est ainsi caractérisé par un rythme haché, mettant en relief le mot plutôt que le syntagme. Il arrive souvent aussi que les présentateurs [...] émettent les dernières syllabes d'une phrase avec une intensité décroissante telle que l'auditeur ne les perçoit plus, comme si toute l'énergie avait été dépensée au début de l'émission de la phrase et des groupes sur les accents d'insistance » (Léon 1993, 165-166).

Ce « phonostyle » (Léon 1993), qui fait ici référence à une combinaison de variantes de prononciation perçues comme propres à l'activité professionnelle des présentateurs de journaux audiovisuels (cf. Simon/Lacheret 2016, 382) et que l'on peut considérer comme étant bien connu des informateurs en raison de la large diffusion médiatique de ces journaux, pourrait donc avoir aiguillé la perception des informateurs vers les traits prosodiques plus que vers le trait segmental qui devait être testé. Pour ces deux raisons, les syntagmes extraits du texte PFC s'avèrent peu fiables, ce qui explique pourquoi les interprétations proposées dans les trois sous-chapitres reposent principalement sur les résultats touchant aux mots isolés.

Notons cependant que cette concentration sur des mots isolés n'est pas sans poser également certains problèmes méthodologiques : il est en effet possible que les informateurs - en particulier dans les questions reposant sur une comparaison directe de deux stimuli - ne se basent pas sur leurs perceptions (implicites) dans l'évaluation des oppositions, mais sur des représentations (explicites) de ces oppositions qu'ils ne remarqueraient pas dans des contextes de comparaison moins explicites. Ce problème pourrait par ailleurs avoir été renforcé par la présentation graphique de ces mots dans le cadre du test, pouvant inciter les informateurs à chercher une différence de prononciation qu'ils n'entendraient 
peut-être pas dans un contexte plus naturel et sans influence de la graphie (cf. 3.5.2). En d'autres termes, l'effet de la présentation de mots isolés par paires, combinée à la présentation graphique des mots testés, est susceptible d'exacerber ou de mettre l'accent sur les différences qui passeraient peut-être inaperçues si le trait était présenté dans un mot ne faisant pas partie d'une paire minimale et/ou n'étant pas mis justement en contraste. Ce problème a été notamment remarqué, en Suisse romande, pour la différence entre synérèse et diérèse et l'opposition /õ̃/ : / $\tilde{\varepsilon} /$ (cf. 6.2.4 ; 6.2.5), au Québec, pour le relâchement des voyelles /i, y, u/ (cf. 6.3.4) et, à Paris, pour l'ensemble des quatre traits (cf. 6.1.1-6.1.4). Soulignons cependant que si cet effet est sensible, il ne semble pas fausser les résultats globaux. En effet, il ne touche que les questions 2 (= prononciation idéale des présentateurs de journaux télévisés) et 3 (= prononciation à enseigner dans les cours de FLE) des tests de perception. Or, dans la première question (= évaluation sans mention explicite d'un contexte pour la norme), dans laquelle chaque stimulus a été au contraire testé séparément, les réponses présentent tout de même des tendances similaires aux questions 2 et 3 .

Mentionnons d'ailleurs que cette forte similitude entre les résultats touchant aux trois types de questions est relativement systématique dans les trois tests de perception. Trois explications peuvent être invoquées pour expliquer ce constat frappant : premièrement, au niveau méthodologique, il est possible que la répétition des stimuli pour chacune des questions ait eu un effet notable sur les réponses, en ce sens que dans les questions apparaissant plus tard dans le test, les évaluations auront éventuellement été effectuées sans réelle prise en compte du type de question. Deuxièmement, au niveau méthodologique également, il est possible que la conception du test n'ait finalement rendu compte que des norme(s) prescriptive(s) des représentations et non des normes descriptives des productions (cf. Pustka/Chalier/Jansen 2017, 102-103). Troisièmement, au niveau théorique, il est également envisageable que les différences entre les types de normes n'existent pas réellement - ce qui plaiderait en faveur de la conception d'une norme unique, stable et indépendante de la situation de communication. Il s'agit là de trois explications possibles à un problème qui devra encore être analysé dans de futures études. 